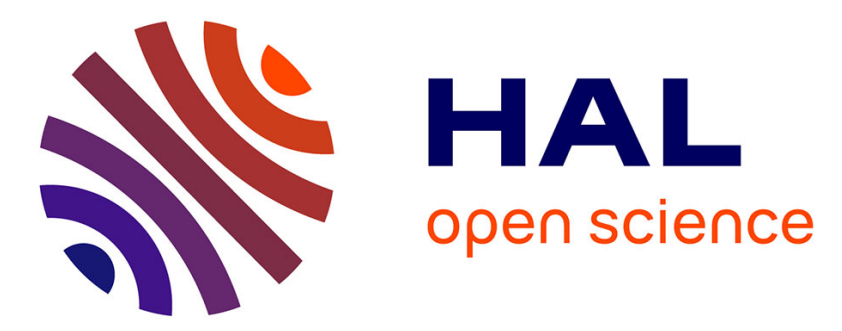

\title{
Godunov-type schemes with an inertia term for unsteady full Mach number range flow calculations
}

Yann Moguen, Simon Delmas, Vincent Perrier, Pascal Bruel, Erik Dick

\section{To cite this version:}

Yann Moguen, Simon Delmas, Vincent Perrier, Pascal Bruel, Erik Dick. Godunov-type schemes with an inertia term for unsteady full Mach number range flow calculations. Journal of Computational Physics, 2015. hal-02362382

\section{HAL Id: hal-02362382 \\ https://hal-univ-pau.archives-ouvertes.fr/hal-02362382}

Submitted on 13 Nov 2019

HAL is a multi-disciplinary open access archive for the deposit and dissemination of scientific research documents, whether they are published or not. The documents may come from teaching and research institutions in France or abroad, or from public or private research centers.
L'archive ouverte pluridisciplinaire HAL, est destinée au dépôt et à la diffusion de documents scientifiques de niveau recherche, publiés ou non, émanant des établissements d'enseignement et de recherche français ou étrangers, des laboratoires publics ou privés. 


\title{
Godunov-type schemes with an inertia term for unsteady full Mach number range flow calculations
}

\author{
Yann Moguen ${ }^{\mathrm{a}, *}$, Simon Delmas $^{\mathrm{a}}$, Vincent Perrier $^{\mathrm{b}}$, Pascal Bruel $^{\mathrm{c}}$ \\ and Erik Dick ${ }^{\mathrm{d}}$ \\ ${ }^{a}$ Université de Pau et des Pays de l'Adour LMAP UMR 5142 and Inria CAGIRE Team- \\ Avenue de l'Université - 64013 Pau, France \\ ${ }^{\mathrm{b}}$ Inria CAGIRE Team, Université de Pau et des Pays de l'Adour LMAP UMR 5142 - \\ Avenue de l'Université - 64013 Pau, France \\ ${ }^{\mathrm{c}}$ CNRS, Université de Pau et des Pays de l'Adour LMAP UMR 5142 and Inria CAGIRE \\ Team - Avenue de l'Université - 64013 Pau, France \\ ${ }^{\mathrm{d}}$ Ghent University - Department of Flow, Heat and Combustion Mechanics, \\ Sint-Pietersnieuwstraat, $41-9000$ Gent, Belgium.
}

\begin{abstract}
An inertia term is introduced in the $\mathrm{AUSM}^{+}$-up scheme. The resulting scheme, called AUSM-IT (IT for Inertia Term), is designed as an extension of the $\mathrm{AUSM}^{+}$-up scheme allowing for full Mach number range calculations of unsteady flows including acoustic features. In line with the continuous asymptotic analysis, the AUSM-IT scheme satisfies the conservation of the discrete linear acoustic energy at first order in the low Mach number limit. Its capability to properly handle low Mach number unsteady flows, that may include acoustic waves or discontinuities, is numerically illustrated. The approach for building the AUSM-IT scheme from the $\mathrm{AUSM}^{+}$-up scheme is applicable to any other Godunov-type scheme.
\end{abstract}

Key words: All Mach number schemes, AUSM schemes, Godunov-type schemes, Low Mach number, Momentum interpolation

\section{Introduction}

Convective and acoustic waves may propagate together in compressible flows, at time and space scales that may be very different, and with possible interactions.

\footnotetext{
* Corresponding author. Tel.: +33 559407552 .

Email address: yann.moguen@univ-pau.fr(Yann Moguen).
} 
Design of numerical methods able to handle properly these phenomena remains a challenging task, even if viscous effects are neglected. With a co-located arrangement of the unknowns, accuracy and robustness of the numerical method depend on the way of interpolation on the cell or element faces. Two broad categories of methods can be identified, according to the equations they are derived from: (1) Methods solving a Riemann problem at each face by using characteristic equations (these methods are referred to as Godunov-type schemes in the present study) ; (2) Momentum interpolation methods, derived from the momentum equation. In our opinion, the relations between these two approaches merit investigation in order to improve their respective capabilities.

The difficulties arising at low Mach number when Godunov-type schemes are used have been widely studied, mainly for steady calculations (see e.g. [1-3,11]). Denoting by $\mathrm{M}_{\mathrm{r}}$ a reference Mach number in the flow, it has been recognized that avoiding the checkerboard decoupling problem needs a $1 / \mathrm{M}_{\mathrm{r}}^{2}-$ scaling of the pressure gradient term in the face velocity or the face mass flux. This $1 / \mathrm{M}_{\mathrm{r}}^{2}-$ scaling implies that the thermodynamic and the acoustic pressures are constant in space at the convective scale, which conforms to the continuous asymptotic analysis, provided that suitable boundary conditions are adopted (see e.g. [3,6]). For AUSM-type schemes, it was shown by Dellacherie [1] that the $1 / \mathrm{M}_{\mathrm{r}}^{2}$-scaling is also necessary for avoiding spurious acoustic waves when starting from so-called well-prepared initial conditions. However, there is experimental evidence that this property does not guarantee accurate calculation of acoustic propagation in low Mach number flows. In our earlier work [15], it was thus observed that for unsteady calculations of low Mach number flows, the presence of the time-step in the pressure-velocity coupling coefficient of the face velocity, as for momentum interpolation, is beneficial. Based on this observation, an improvement of the $\mathrm{AUSM}^{+}$-up scheme was then proposed in [14], by mimicking the pressure-velocity coupling of the momentum interpolation. However, we noted that the quality of the momentum interpolation, if properly defined for unsteady calculations in a Rhie-Chow-like manner (see [15-17]), was not reached for some tests at low Mach number. Improvement of predictions for unsteady low Mach number flows by the $\mathrm{AUSM}^{+}$-up scheme and the related SLAU scheme (Simple Low Dissipative AUSM) by introduction of Strouhal number dependence in the coefficient of the pressure dissipation term in the mass flux expression was also obtained by Sachdev et al. [19]. These authors demonstrated that the scaling of the coupling coefficient has to be quite different for steady low Mach number problems and for unsteady low Mach number problems. By changing the scaling, they proved significant improvement with the $\mathrm{AUSM}^{+}$-up scheme for unsteady low Mach number problems with hydrodynamic coupling between velocity and pressure (vortex propagation) and with acoustic coupling (propagation of a pressure oscillation and propagation of weak shocks and expansion fans). They also illustrated oscillatory behavior of the SLAU method for steady low Mach number flows. However, it remains unclear how to combine the different scaling factors and, for instance, to ensure that the correct steady scaling is obtained for the solution of a steady low Mach number problem calculated with an unsteady 
method. A similar remark holds for our own work [14]. A particular problem with the $\mathrm{AUSM}^{+}$-up method is that the damping by the pressure difference term in the mass flux expression which is appropriate for steady low Mach number flow is too high for propagation of smooth acoustic signals in unsteady low Mach number flows. On the other hand, as observed by Sachdev et al. [19], the dissipation is too low for propagation of acoustic discontinuities (low Mach number Riemann problems). So, it becomes very delicate to tune the pressure dissipation such that it functions properly for the different types of low Mach number flows. Too low pressure dissipation in the mass flux definition of the SLAU method for simulation of propagation of discontinuities in low Mach number flows was also remarked by Shima [20]. He proved that it is possible to eliminate oscillations by increasing the coefficient of the pressure dissipation term in the mass flux definition by a sensor for wiggles. Of course, the larger dissipation smears somewhat the discontinuities. The conclusion is that methods that rely on tuning of the coefficient of the pressure dissipation term in the mass flux definition in AUSM-type discretizations are very delicate and certainly have not reached maturity yet.

Observing that an inertia term is present in the face velocity expression by the momentum interpolation, and missing in the face velocity expression by the scheme proposed in [14], we propose in the present study to introduce this inertia term in the face velocity of Godunov-type schemes. The momentum interpolation is used as a guideline to accommodate this term. The resulting pressure-velocity coupling exhibits then the suitable $1 / \mathrm{M}_{\mathrm{r}}^{2}$-scaling for low Mach number steady calculations. In the unsteady case, the pressure-velocity coupling exhibits also the proper Mach number scaling and time-step dependence, identified in $[14,15]$. Moreover, the inertia term is introduced such that the steady state, if it exists, does not depend on the time-step.

As pointed out in [1,2], an asymptotic property providing insights for the design of Godunov-type schemes that remain accurate at low Mach number is the linear acoustic energy conservation in the low Mach number regime, which holds if periodic boundary conditions are adopted. This property is used in the present study as a guideline to assess the proper way of inertia term interpolation, as well as the proper way of pressure interpolation, in order to enforce the acoustic energy conservation at the discrete level.

The key point is that, if the acoustic component of the pressure is centrally interpolated in the low Mach number limit, the presence of the inertia term in the face velocity enforces acoustic energy conservation at the discrete level. More precisely, the $1 / \mathrm{M}_{\mathrm{r}}-$ scaling of the numerical dissipation that arises from the spatial discretization of the linear acoustic wave equation, is thus counterbalanced. Conservation of acoustic energy is clearly a prerequisite for accurate calculation of unsteady low Mach number flows including acoustic features. 


\section{Foundation of Godunov-type schemes on characteristic equations}

In this section, the Mach number scaling of the pressure gradient term in the face velocity expression of Godunov-type schemes is examined in the light of the characteristic equations from which these schemes are drawn.

Reference pressure $p_{\mathrm{r}}$, density $\varrho_{\mathrm{r}}$ and velocity $v_{\mathrm{r}}$ thought of as a convective quantity, are introduced. A reference Mach number is then defined as $\mathrm{M}_{\mathrm{r}}=v_{\mathrm{r}} / \sqrt{p_{\mathrm{r}} / \varrho_{\mathrm{r}}}$. Reference length $l_{\mathrm{r}}$ and duration $t_{\mathrm{r}}$, thought of as a convective quantity, are also considered, as well as a reference Strouhal number, $\mathrm{St}_{\mathrm{r}}=\left(l_{\mathrm{r}} / v_{\mathrm{r}}\right) / t_{\mathrm{r}}$. Notice that it is possible to choose the reference length $l_{\mathrm{r}}$ as $t_{\mathrm{r}} \sqrt{p_{\mathrm{r}} / \varrho_{\mathrm{r}}}$, which is an acoustic length. Then, the reference Strouhal and Mach numbers are related by $\mathrm{St}_{\mathrm{r}}=1 / \mathrm{M}_{\mathrm{r}}$. Here however, the possibility is left open for another choice of reference duration, so that we will work with the reference Strouhal number $\mathrm{St}_{\mathrm{r}}$. Associated with the Euler equations in dimensional form,

$$
\begin{array}{r}
\partial_{t} \varrho+\nabla \cdot(\varrho \boldsymbol{v})=0, \\
\partial_{t}(\varrho \boldsymbol{v})+\nabla \cdot(\varrho \boldsymbol{v} \otimes \boldsymbol{v})+\nabla p=0, \\
\partial_{t}(\varrho E)+\nabla \cdot(\varrho H \boldsymbol{v})=0, \\
E=e+\frac{1}{2}\|\boldsymbol{v}\|^{2}, \\
\varrho H=\varrho E+p, \\
\varrho e=\frac{p}{\gamma-1},
\end{array}
$$

the dimensionless 1-D characteristic equations read as ${ }^{1}$ :

$$
\begin{aligned}
\mathrm{d} v-\frac{1}{\mathrm{M}_{\mathrm{r}}} \frac{\mathrm{d} p}{\varrho c}=0 \quad \text { on } \quad \operatorname{St}_{\mathrm{r}} \mathrm{d}_{t} x=v-\frac{c}{\mathrm{M}_{\mathrm{r}}}, \\
\mathrm{d} \varrho-\frac{1}{c^{2}} \mathrm{~d} p=0 \quad \text { on } \quad \operatorname{St}_{\mathrm{r}} \mathrm{d}_{t} x=v, \\
\mathrm{~d} v+\frac{1}{\mathrm{M}_{\mathrm{r}}} \frac{\mathrm{d} p}{\varrho c}=0 \quad \text { on } \quad \operatorname{St}_{\mathrm{r}} \mathrm{d}_{t} x=v+\frac{c}{\mathrm{M}_{\mathrm{r}}} .
\end{aligned}
$$

A prototypical AUSM-type interpolation, which can be thought of as a common starting point for the different variants presented by Liou et al. [10-12], can be derived from the characteristic equations (2) and (3) as follows. From Eq. (3), where the inertia term is omitted,

$$
\partial_{x} v^{+}+\frac{1}{\mathrm{M}_{\mathrm{r}} \varrho c} \partial_{x} p^{+}=0 .
$$

$\overline{1}$ To ease the reading of the following derivations, we keep the same notations for dimensional and non-dimensional quantities. 
The superscript + indicates here the propagating direction. With $\Delta x=x_{i+1}-x_{i}$, Eq. (4) is discretized on the control volume $\left[x_{i}, x_{i+1}\right]$ into

$$
\frac{v_{i+1}^{+}-v_{i}^{+}}{\Delta x}+\frac{1}{\mathrm{M}_{\mathrm{r}}(\varrho c)_{i+1 / 2}} \frac{p_{i+1}^{+}-p_{i}^{+}}{\Delta x}=0,
$$

from which we obtain

$$
\frac{v_{i}^{+}+v_{i+1}^{+}}{2}=v_{i}^{+}-\frac{1}{2} \frac{1}{\mathrm{M}_{\mathrm{r}}(\varrho c)_{i+1 / 2}}\left(p_{i+1}^{+}-p_{i}^{+}\right) .
$$

Let

$$
v_{i+1 / 2}^{+}=\frac{v_{i}^{+}+v_{i+1}^{+}}{2} .
$$

Then,

$$
v_{i+1 / 2}^{+}=v_{i}^{+}-\frac{1}{2} \frac{1}{\mathrm{M}_{\mathrm{r}}(\varrho c)_{i+1 / 2}}\left(p_{i+1}^{+}-p_{i}^{+}\right) .
$$

Similarly, setting

$$
v_{i+1 / 2}^{-}=\frac{v_{i}^{-}+v_{i+1}^{-}}{2}
$$

one has from Eq. (2), where the inertia term is omitted,

$$
v_{i+1 / 2}^{-}=v_{i+1}^{-}-\frac{1}{2} \frac{1}{\mathrm{M}_{\mathrm{r}}(\varrho c)_{i+1 / 2}}\left(p_{i+1}^{-}-p_{i}^{-}\right) .
$$

Then, setting

$$
p_{i}=\frac{p_{i}^{+}+p_{i}^{-}}{2} \quad, \quad p_{i+1}=\frac{p_{i+1}^{+}+p_{i+1}^{-}}{2}
$$

and

$$
v_{i+1 / 2}=\frac{v_{i+1 / 2}^{+}+v_{i+1 / 2}^{-}}{2},
$$

one obtains from Eqs. (5)-(6):

$$
v_{i+1 / 2}=\frac{v_{i}^{+}+v_{i+1}^{-}}{2}-\frac{1}{2} \frac{1}{\mathrm{M}_{\mathrm{r}}(\varrho c)_{i+1 / 2}}\left(p_{i+1}-p_{i}\right) .
$$

In the derivation of the face velocity (7), inertia terms are omitted in the acoustic characteristic equations (2) and (3). As a consequence of this omission and of the acoustic origin of expression (7), the Mach number scaling of the pressure 
gradient term is $1 / \mathrm{M}_{\mathrm{r}}$. Thus, the pressure gradient term can be thought of as an acoustic quantity that operates, in the low Mach number regime, at the large acoustic length scale solely. However, to fix the checkerboard decoupling at low Mach number, which is a problem that may appear at the small convective length scale if a co-located arrangement is used, the low Mach number scaling of the pressure gradient term should be $1 / \mathrm{M}_{\mathrm{r}}^{2}$ at the small convective length scale. This is the reason of the introduction of a scaling function which belongs to $O\left(\mathrm{M}_{\mathrm{r}}\right)$, and modifies the $1 / \mathrm{M}_{\mathrm{r}}$-scaling of the pressure gradient term into the $1 / \mathrm{M}_{\mathrm{r}}^{2}-$ scaling at low Mach number, as in $[3,8,11,18,21]$ for example. However, we observe that the $1 / \mathrm{M}_{\mathrm{r}}^{2}$-scaling of the pressure gradient term in the face velocity expression is obvious from the momentum equation (1b). Following further this approach, the inertia term is introduced in the face velocity expression according to its relation to the pressure gradient term in the momentum equation (1b). This yields:

$$
v_{i+1 / 2}=\frac{v_{i}+v_{i+1}}{2}-\frac{1}{2(\varrho c)_{i+1 / 2} \mathrm{M}_{\mathrm{r}}^{2}}\left(p_{i+1}-p_{i}\right)-\frac{\mathrm{St}_{\mathrm{r}} \Delta x}{2 c_{i+1 / 2} \mathrm{M}_{\mathrm{r}}} \partial_{t} v_{i+1 / 2}
$$

For the pressure at the face, the central interpolation is chosen in the low Mach number regime:

$$
p_{i+1 / 2}=\frac{p_{i}+p_{i+1}}{2} .
$$

Arguments for the choice of the scheme by Eqs. (8)-(9) is the matter of the next section.

\section{Role of inertia term in acoustic energy conservation at low Mach number}

In this section, we concentrate on the benefits, at low Mach number, of the presence of an inertia term in the scheme expressed by Eqs. (8) and (9). The linear acoustic energy behavior obtained when this scheme is used, is examined through asymptotic expansions and modified equations.

\subsection{Basics of two-scale low Mach number continuous asymptotics}

The flow is considered in any dimension $d=1,2$ or 3 . In the low Mach number regime, a variable relevant to reveal the behavior of the flow at the large acoustic length scale is introduced as

$$
\boldsymbol{\xi}=\mathrm{M}_{\mathrm{r}} \boldsymbol{x}
$$


Then, we assume that the pressure can be expanded as

$$
p\left(\boldsymbol{x}, t, \mathrm{M}_{\mathrm{r}}\right)=\sum_{n=0}^{N} \mathrm{M}_{\mathrm{r}}^{n} p^{(n)}(\boldsymbol{x}, \boldsymbol{\xi}, t)+o\left(\mathrm{M}_{\mathrm{r}}^{N}\right), \quad N=0,1,2
$$

with similar expansions for density $\varrho$ and velocity $\boldsymbol{v}$. After substitution of these expansions into the Euler equations (1) non-dimensionalized with the reference quantities introduced in Sec. 2, one obtains ${ }^{2}$ :

$$
\begin{aligned}
& \mathrm{St}_{\mathrm{r}} \partial_{t} \widetilde{\boldsymbol{v}^{(0)}}+\frac{1}{\widetilde{\varrho^{(0)}}} \nabla_{\boldsymbol{\xi}} p^{(1)}=0, \\
& \mathrm{St}_{\mathrm{r}} \partial_{t} p^{(1)}+\gamma p^{(0)} \nabla_{\boldsymbol{\xi}} \cdot \widetilde{\boldsymbol{v}^{(0)}}=0 .
\end{aligned}
$$

At the acoustic length scale, Eqs. (10a) and (10b) can be interpreted as momentum and energy equations, respectively. These equations constitute the first-order wave equation, as evidenced by deriving from them a non-linear equation for acoustic wave propagation,

$$
\partial_{t t} p^{(1)}-\nabla \cdot\left(c_{0}^{2} \nabla_{\boldsymbol{\xi}} p^{(1)}\right)=0
$$

where $c_{0}=c_{0}(\boldsymbol{\xi}, t)=\sqrt{\gamma p^{(0)}(t) / \widetilde{\varrho^{(0)}}(\boldsymbol{\xi})}$. Thus, considered at the acoustic length scale, $\widetilde{\boldsymbol{v}^{(0)}}$ and $p^{(1)}$ are identified as acoustic variables.

\subsection{First-order modified equations at the acoustic scale}

How the scheme by Eqs. (8) and (9) operates on the acoustic variables identified in Sec. 3.1 is studied in this section. Considering first the 1-D case, velocity $\widetilde{v^{(0)}}$ and pressure $p^{(1)}$ are inserted into Eq. (8), as

$$
\widetilde{v_{i+1 / 2}^{(0)}}=\frac{\widetilde{v_{i}^{(0)}}+\widetilde{v_{i+1}^{(0)}}}{2}-\frac{1}{2\left(\widetilde{\varrho^{(0)}} c^{(0)}\right)_{i+1 / 2} \mathrm{M}_{\mathrm{r}}^{2}}\left(p_{i+1}^{(1)}-p_{i}^{(1)}\right)-\frac{\mathrm{St}_{\mathrm{r}} \Delta x}{2 c_{i+1 / 2}^{(0)} \mathrm{M}_{\mathrm{r}}} \widetilde{\partial_{t} v_{i+1 / 2}^{(0)}},
$$

where it is supposed that $\Delta \xi=\mathrm{M}_{\mathrm{r}} \Delta x$, which means that the grid size $\Delta x$ is of the order of the small convective length scale. From Eq. (9), central interpolation of the acoustic pressure is adopted:

$$
p_{i+1 / 2}^{(1)}=\frac{p_{i}^{(1)}+p_{i+1}^{(1)}}{2} .
$$

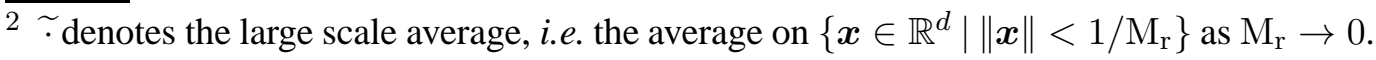
This average operation allows to separate features at the acoustic length scale from those at the convective length scale through the so-called sublinear growth lemma; see e.g. Klein [6] or Meister [13] for details. 
Suppose that $\widetilde{\varrho^{(0)}}$ and $c^{(0)}$ are constant in space. With the central interpolation (12), at the first order, the modified equation of Eq. (10a) on a Cartesian grid is identical to the original equation. If $\Delta x$ is equal in each dimension, the same result is obtained for dimension $d$ higher than 1. Similarly, with the obvious multi-dimensional extension of interpolation (11), the first-order modified equation of Eq. (10b) is obtained for dimension $d=1,2$ or 3 on a uniform Cartesian grid, as

$$
\mathrm{St}_{\mathrm{r}} \partial_{t} p^{(1)}+\gamma p^{(0)} \nabla_{\boldsymbol{\xi}} \cdot \widetilde{\boldsymbol{v}^{(0)}}=\frac{c^{(0)} \Delta x}{2 \mathrm{M}_{\mathrm{r}}}\left(\nabla_{\boldsymbol{\xi}} \cdot \nabla_{\boldsymbol{\xi}} p^{(1)}+\varrho^{(0)} \mathrm{St}_{\mathrm{r}} \partial_{t} \nabla_{\boldsymbol{\xi}} \cdot \widetilde{\boldsymbol{v}^{(0)}}\right)
$$

With Eq. (10a), the right-hand side of Eq. (13) is zero, so that the first-order modified equations of Eqs. (10) are identical to the initial first-order wave equation. The role of the inertia term regarding this property is examined in the following section. Note that the coefficient $c^{(0)} \Delta x /\left(2 \mathrm{M}_{\mathrm{r}}\right)$ in Eq. (13) is equivalent, in the asymptotic framework adopted here, to the numerical viscosity coefficient of Godunov-type schemes for the linear wave equation identified by Dellacherie [1].

\subsection{Inertia term requirement for acoustic energy conservation}

In this section, $\widetilde{\varrho^{(0)}}$ and $c^{(0)}$ are assumed to be constant in space and time. The linear acoustic energy on the unit torus $\mathbb{T}=(\mathbb{R} /(2 \pi \mathbb{Z}))^{d}(d=1,2$ or 3$)$ at low Mach number is

$$
E_{a}=\int_{\mathbb{T}}\left[\frac{1}{2} \widetilde{\varrho^{(0)}}\left\|\widetilde{\boldsymbol{v}^{(0)}}\right\|^{2}+\frac{1}{2} \frac{\left(p^{(1)}\right)^{2}}{\widetilde{\varrho^{(0)}\left(c^{(0)}\right)^{2}}}\right] .
$$

Note that

$$
\mathrm{d}_{t} E_{a}=\widetilde{\varrho^{(0)}} \int_{\mathbb{T}} \widetilde{\boldsymbol{v}^{(0)}} \cdot \partial_{t} \widetilde{\boldsymbol{v}^{(0)}}+\frac{1}{\widetilde{\varrho^{(0)}\left(c^{(0)}\right)^{2}}} \int_{\mathbb{T}} p^{(1)} \partial_{t} p^{(1)}
$$

Then, on the one hand, with Eqs. (10a) and (10b),

$$
\mathrm{d}_{t} E_{a}=0 .
$$

On the other hand, with first-order modified equations (10a) and (13), Eq. (15) holds too, so that the conservation of acoustic energy for the initial continuous system of equations is retrieved at the first order.

Moreover, one obtains from Eq. (14) and from the first-order modified equations associated with Eqs. (10a)-(10b) that, without an inertia term, acoustic energy is dissipated with the following decrease rate:

$$
\mathrm{d}_{t} E_{a}=-\frac{\Delta x}{2 \mathrm{St}_{\mathrm{r}} \widetilde{\varrho^{(0)}} c^{(0)} \mathrm{M}_{\mathrm{r}}} \int_{\mathbb{T}}\left\|\nabla_{\boldsymbol{\xi}} p^{(1)}\right\|^{2} \leq 0 .
$$


Therefore, as included in Eq. (8), the inertia term compensates the acoustic energy dissipation at the acoustic length scale and at the first order. The conservation of acoustic energy at low Mach number, which is satisfied by the continuous system of equations (10a)-(10b), is thus ensured at the discrete level.

Note that when third-order modified equations associated with Eqs. (10a)-(10b) are derived instead of first-order equations, one readily obtains:

$$
\mathrm{d}_{t} E_{a}=\frac{\mathrm{M}_{\mathrm{r}}(\Delta x)^{3}}{48 \mathrm{St}_{\mathrm{r}} \varrho^{(0)} c^{(0)}} \int_{\mathbb{T}}\left(\nabla_{\boldsymbol{\xi}} \cdot \nabla_{\boldsymbol{\xi}} p^{(1)}\right)^{2}
$$

Even if this quantity belongs to $O\left(\mathrm{M}_{\mathrm{r}}^{2}\right)$ if the Strouhal number is defined with an acoustic reference length (see Sec. 2), some risk, in particular for high-order computations, arises from its positivity. It is thus advisable to introduce some Mach number-dependent upwinding in the interpolation of the convective terms in Eq. (11). In the present study, this is carried out through the interface Mach number suggested by Liou [10], as presented in the next section.

\section{The AUSM-IT scheme}

In this section, we present a Godunov-type scheme with an inertia term, called the AUSM-IT scheme (IT for Inertia Term). It is designed such as to satisfy the following properties:

(1) If the local Mach number exceeds unity, the $\mathrm{AUSM}^{+}$-up scheme by Liou [11] is retrieved ; this allows to inherit from all the abilities of this well-established scheme in supersonic regime ${ }^{3}$.

(2) When $M_{r}$ goes to zero, the face velocity and face pressure tend to the expressions given in Eqs. (8) and (9) ; this allows to satisfy the property of acoustic energy conservation at the first order, presented in the previous section.

The AUSM-IT scheme is defined by the following face velocity and face pressure expressions.

- Face velocity:

$\overline{3}$ The reader interested by a recent development of the $\mathrm{AUSM}^{+}$-up scheme to avoid difficulties related to the global cut-off problem is referred to $\mathrm{Li}$ and $\mathrm{Gu}$ [9]. 


$$
\begin{aligned}
& v_{1 / 2}=c_{1 / 2} M_{1 / 2}-\frac{K_{p}}{\varrho c_{1 / 2} f_{c}\left(M_{0}\right)} \max \left\{1-\sigma \bar{M}^{2}, 0\right\} \Delta p \\
& \quad-\frac{K_{I}}{c_{1 / 2} f_{c}\left(M_{0}\right)} \max \left\{1-\sigma \bar{M}^{2}, 0\right\} \Delta x \partial_{t} v_{1 / 2} \quad \text { (16) } \\
& M_{1 / 2}=f_{M}^{+}\left(M_{\mathrm{L}}\right)+f_{M}^{-}\left(M_{\mathrm{R}}\right) \\
& f_{M}^{ \pm}(m)=\left\{\begin{array}{l}
\frac{1}{2}(m \pm|m|), \\
\pm \frac{1}{4}(m \pm 1)^{2} \pm \frac{1}{8}\left(m^{2}-1\right)^{2}, \quad|m|<1
\end{array}\right. \\
& \bar{\varrho}=\frac{\varrho_{\mathrm{L}}+\varrho_{\mathrm{R}}}{2} \\
& f_{c}\left(M_{0}\right)=M_{0}\left(2-M_{0}\right), M_{0}^{2}=\min \left\{1, \max \left\{\bar{M}^{2}, M_{\infty}^{2}\right\}\right\} \\
& \bar{M}^{2}=\frac{\left(V_{n}^{+}\right)^{2}+\left(V_{n}^{-}\right)^{2}}{2 c_{1 / 2}^{2}} \\
& c_{1 / 2}=\min \left\{\tilde{c}_{\mathrm{L}}, \tilde{c}_{\mathrm{R}}\right\}, \tilde{c}_{\mathrm{L}}=\left(c^{\star}\right)^{2} / \max \left\{c^{\star}, V_{n}^{+}\right\}, \tilde{c}_{\mathrm{R}}=\left(c^{\star}\right)^{2} / \max \left\{c^{\star},-V_{n}^{-}\right\} \\
& \left(c^{\star}\right)^{2}=\frac{2(\gamma-1)}{\gamma+1} H \\
& 0 \leq K_{p} \leq 1,0 \leq K_{I} \leq 1, \sigma \leq 1 ; \text { typically: } K_{p}=K_{I}=0.25, \sigma=1
\end{aligned}
$$

- Face pressure:

$$
p_{1 / 2}=f_{p, \alpha}^{+}\left(M_{\mathrm{L}}\right) p_{\mathrm{L}}+f_{p, \alpha}^{-}\left(M_{\mathrm{R}}\right) p_{\mathrm{R}}
$$

where

$$
\begin{aligned}
& f_{p, \alpha}^{ \pm}(m)= \begin{cases}\frac{1}{2}(1 \pm \operatorname{sign}(m)) & ,|m| \geq 1 \\
\frac{1}{4}(m \pm 1)^{2}(2 \mp m) \pm \alpha m\left(m^{2}-1\right)^{2} & ,|m|<1\end{cases} \\
& \alpha=\frac{3}{16}\left[-4+5\left(f_{c}\left(M_{0}\right)\right)^{2}\right]
\end{aligned}
$$

- Convected quantities: $\varrho, \varrho v, \varrho H$, upwinded as proposed for the $\mathrm{AUSM}^{+}$-up scheme by Liou [11], e.g.,

$$
\varrho= \begin{cases}\varrho_{\mathrm{L}}, & \text { if } M_{1 / 2}-\frac{K_{p}}{\bar{\varrho} c_{1 / 2}^{2} f_{c}\left(M_{0}\right)} \max \left\{1-\sigma \bar{M}^{2}, 0\right\} \Delta p \geq 0 \\ \varrho_{\mathrm{R}}, & \text { else }\end{cases}
$$

Remark 1. In Eq. (16), the exact form of the term $\partial_{t} v_{i+1 / 2}$ depends on the time integration method and is not a specific feature of the AUSM-IT scheme. 
Remark 2. The proposed AUSM-IT scheme is an extension of the $\mathrm{AUSM}^{+}$-up scheme such that the $\mathrm{AUSM}^{+}$-up scheme is retrieved when the face pressure is expressed with $K_{v}=0$ and when the inertia term is excluded by taking $K_{I}=0$ in Eq. (16).

Remark 3. Using the AUSM-IT scheme for steady computations is straightforward, since in this case the inertia term $\partial_{t} v_{1 / 2}$ in Eq. (16) vanishes. The scheme then reduces to the $\mathrm{AUSM}^{+}$-up scheme. Notice that the AUSM-IT scheme ensures timestep independence of the steady state. As shown in $[15,16]$, this property is beneficial for steady as well as unsteady computations.

\section{Numerical experiments}

The presented test cases are chosen in order to illustrate the full Mach number range capability of the proposed AUSM-IT scheme. The tests allow to assess the role of the inertia term in the face velocity definition, and to compare the performance with other schemes: $\mathrm{AUSM}^{+}$[10], $\mathrm{AUSM}^{+}$-up [11], SLAU [4,21], 'JCAM 2013' [14], and the momentum interpolation [15,16] (these schemes are described in Appendix A).

In all the test cases considered in the following, the fluid is air with specific heat ratio $\gamma=1.4$.

Dissipation and dispersion errors are used to evaluate the quality of the solution when a reference solution is available. The methodology proposed by Takacs [22] (see also [15]) is employed. Say that $q_{e}$ and $q_{c}$ are exact and computed values of a quantity $q$ under consideration. At a given time $t$, the mean square error can then be defined as

$$
\mathcal{E}^{2}=\frac{1}{N} \sum\left(q_{e}-q_{c}\right)^{2}
$$

where the sum extends over the $N$ nodes of the grid. Further, denoting mean values and variances of the exact and computed quantities by $\bar{q}_{e}, \bar{q}_{c}$ and $\sigma_{e}^{2}, \sigma_{c}^{2}$, the mean square error can be written as

$$
\mathcal{E}^{2}=\sigma_{e}^{2}+\sigma_{c}^{2}+\left(\bar{q}_{e}-\bar{q}_{c}\right)^{2}-2 \operatorname{cov}\left(q_{e}, q_{c}\right)
$$

where $\operatorname{cov}\left(q_{e}, q_{c}\right)$ is the covariance of the two signals. With the correlation coefficient between the two signals,

$$
R\left(q_{e}, q_{c}\right)=\frac{\operatorname{cov}\left(q_{e}, q_{c}\right)}{\sigma_{e} \sigma_{c}}
$$


the error (18) can further be written as

$$
\mathcal{E}^{2}=\left(\bar{q}_{e}-\bar{q}_{c}\right)^{2}+\left(\sigma_{e}-\sigma_{c}\right)^{2}+2 \sigma_{e} \sigma_{c}\left[1-R\left(q_{e}, q_{c}\right)\right]
$$

Non-dimensional measures of dissipation and dispersion errors may then be defined (see [15]) by

$$
\mathcal{E}_{\text {dissipation }}=\frac{\sigma_{c}-\sigma_{e}}{\sigma_{e}}, \mathcal{E}_{\text {dispersion }}=\sqrt{1-R\left(q_{e}, q_{c}\right)} .
$$

Another quantity of interest for assessing the accuracy of the acoustic waves calculations, is the total acoustic energy. Under the linear acoustics assumption, it is defined as

$$
E_{a}=\int_{0}^{L}\left[\frac{1}{2} \varrho_{0}(\delta v)^{2}+\frac{1}{2} \frac{(\delta p)^{2}}{\varrho_{0} c_{0}^{2}}\right],
$$

where $c_{0}=\sqrt{\gamma p_{0} / \varrho_{0}}, \delta p=p-p_{0}$ and $\delta v=v-v_{0}$. Here, $\varrho_{0}, v_{0}$ and $p_{0}$ designate the constant density, velocity and pressure of the uniform 1-D background flow in which the acoustic waves propagate.

\subsection{Low Mach number flows}

\subsubsection{Low Mach number Riemann problem with nearly incompressible initial conditions}

A 1-D Riemann problem with initial conditions given in Table 1 is first considered. The reference Mach number $M_{r}$ is about $10^{-2}$. The computational domain is the interval $[0,1]$ divided into 100 cells of equal length. The time integration is carried out by the first-order explicit forward Euler scheme. The discretization of the inertia term $\partial_{t} v_{1 / 2}$ in Eq. (16) is first-order backward Euler. The numerical method is firstorder accurate in time and space. The convective CFL number is chosen as 0.009, so that the acoustic CFL number is about 0.9. Thus, fluctuations at the acoustic scale can be accurately calculated. At $t=0$, the pressure jump between the right and left states is of order $M_{r}^{2}$, the velocity jump is of order $M_{r}$ and the density jump is zero, so that it belongs to $O\left(\mathrm{M}_{\mathrm{r}}^{2}\right)$ as $\mathrm{M}_{\mathrm{r}} \rightarrow 0$. With such initial conditions, which are often referred to as 'well-prepared' and correspond to a nearly incompressible flow [5], two rarefaction waves propagate in opposite directions as soon as $t>0$ (see Guillard and Murrone [2]).

To examine the role of the pressure gradient term in the face velocity expression when the inertia term is present, the AUSM-IT scheme is considered without pressure dissipation term $\left(K_{p}=0\right.$ and $\left.K_{I}=0.25\right)$, and with $K_{p}=K_{I}=0.25$. We 
set $M_{\infty}=0.01$ (see Eq. (24) in Appendix A), noticing that the exact value of this parameter, if sufficiently small, does not influence significantly the results.

The results of density, velocity, pressure and energy for the AUSM-IT scheme with $K_{p}=K_{I}=0.25$, compared to the exact solution, are shown in Fig. 1 . The results for $K_{p}=0$ and $K_{I}=0.25$ are shown in Fig. 2. The results for the AUSM ${ }^{+}$ scheme $\left(K_{p}=0, K_{I}=0\right)$ are shown in Fig. 3. It is suggested by Liou [11] that shock-tube-type problems in which the pressure distribution is not spatially uniform should be resolved with $f_{c}=1$ when using the $\mathrm{AUSM}^{+}$-up scheme (see Eqs. (23) and (25) in Appendix A). For the Riemann problem under consideration, with nearly incompressible initial conditions, we observed that results by the $\mathrm{AUSM}^{+}$up scheme with $f_{c}$ given by Eq. (24) (see Appendix A) and $f_{c}=1$ are quasiidentical. Thus, only results by the latter choice are shown in Fig. 4.

Comparison of the figures reveals that the AUSM-IT scheme with $K_{p}=0$ and $K_{I}=0.25$, and the $\mathrm{AUSM}^{+}$scheme produce nearly the same results. For $K_{p}=0$, the quality of the AUSM-IT solution clearly deteriorates, since the solution exhibits oscillations near the discontinuities (see Fig. 2) that are absent when $K_{p}=0.25$ (see Fig. 1). We conclude that, in all cases (inertia term present or not), it is beneficial to take $K_{p}=0.25$, thus including the pressure gradient term into the face velocity expression.

The solution calculated by momentum interpolation, shown in Fig. 5, is of bad quality compared to the other schemes. The conclusion is that for Riemann problems at low Mach number, momentum interpolation may fail. 


\begin{tabular}{|c|c|c|c|c|c|}
\hline$\varrho_{\mathrm{L}}\left(\mathrm{kg} / \mathrm{m}^{3}\right)$ & $v_{\mathrm{L}}(\mathrm{m} / \mathrm{s})$ & $p_{\mathrm{L}}(\mathrm{Pa})$ & $\varrho_{\mathrm{R}}\left(\mathrm{kg} / \mathrm{m}^{3}\right)$ & $v_{\mathrm{R}}(\mathrm{m} / \mathrm{s})$ & $p_{\mathrm{R}}(\mathrm{Pa})$ \\
\hline 25 & 0.200 & 10000.00 & 25 & 0.202 & 10000.85 \\
\hline
\end{tabular}

Table 1

Settings for the low Mach number Riemann problem of Sec. 5.1.1.
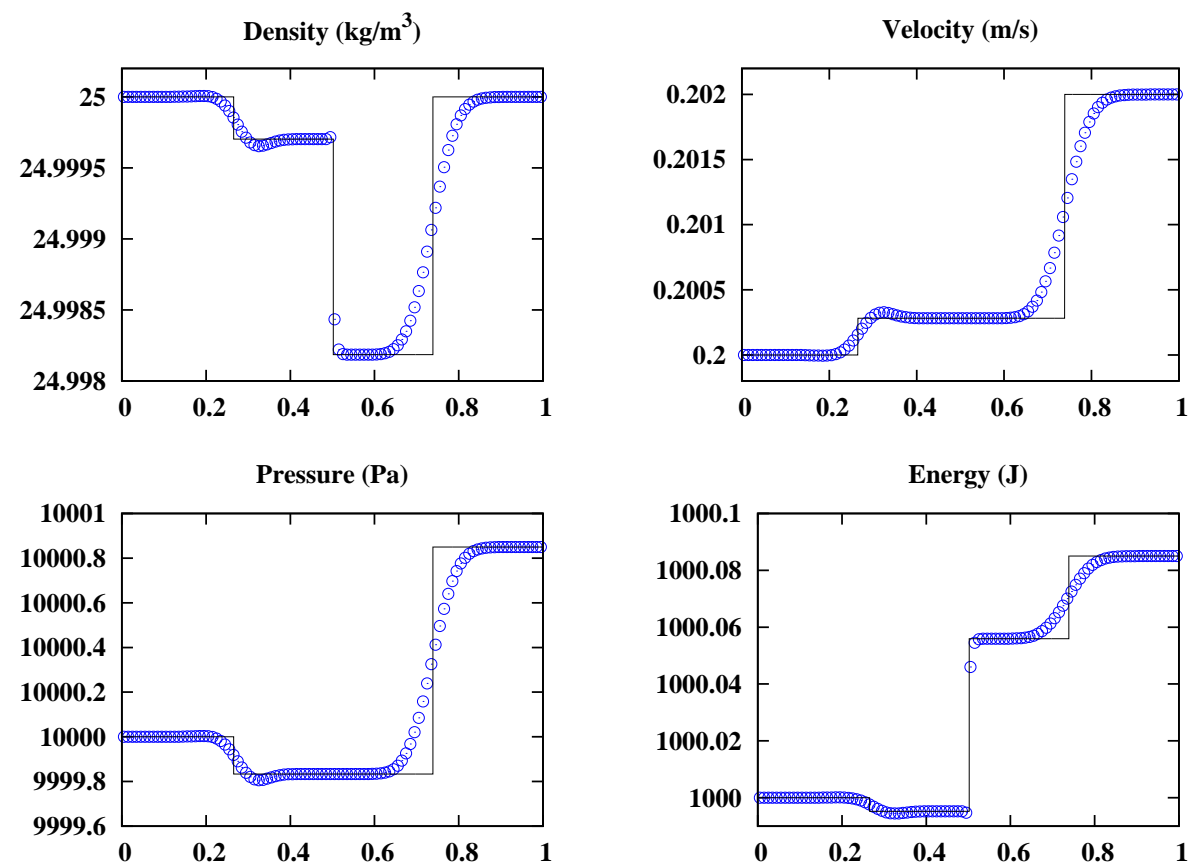

Fig. 1. Low Mach number Riemann problem of Sec. 5.1.1. AUSM-IT scheme with $K_{p}=K_{I}=0.25$ (०) and exact (solid line) solutions at time $t=0.01 \mathrm{~s}$. 

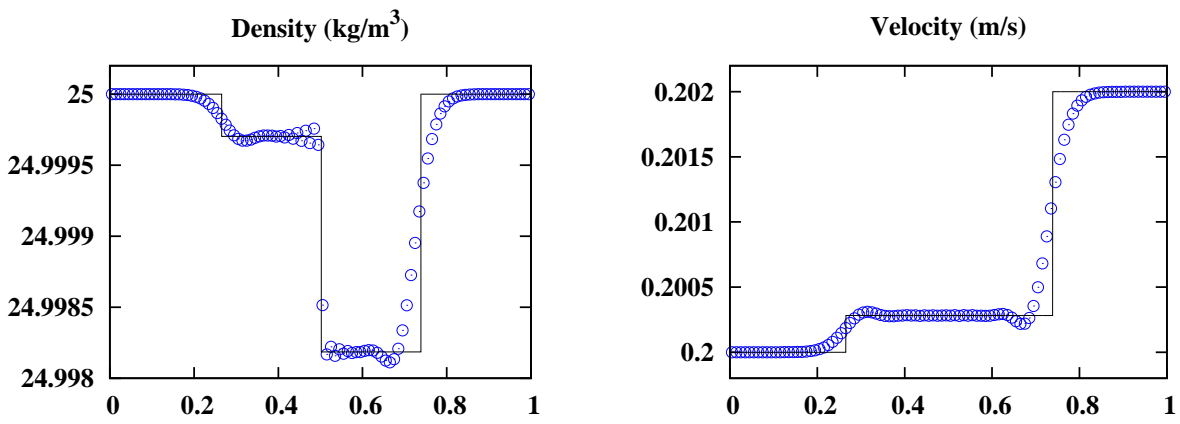

Pressure (Pa)

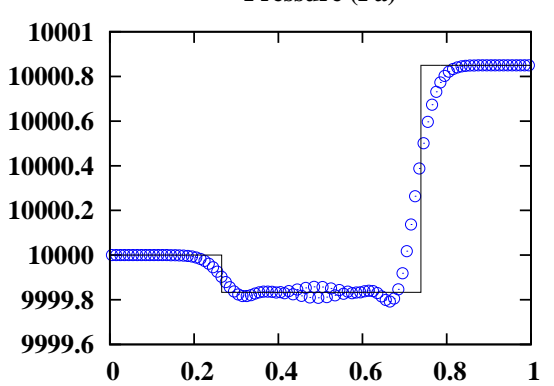

Energy (J)

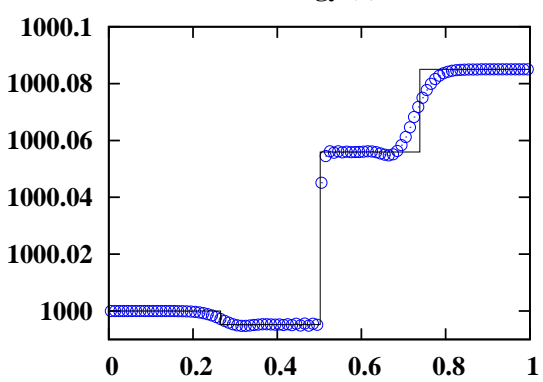

Fig. 2. Low Mach number Riemann problem of Sec. 5.1.1. AUSM-IT scheme with $K_{p}=0$ and $K_{I}=0.25$ (o) and exact (solid line) solutions at time $t=0.01 \mathrm{~s}$.
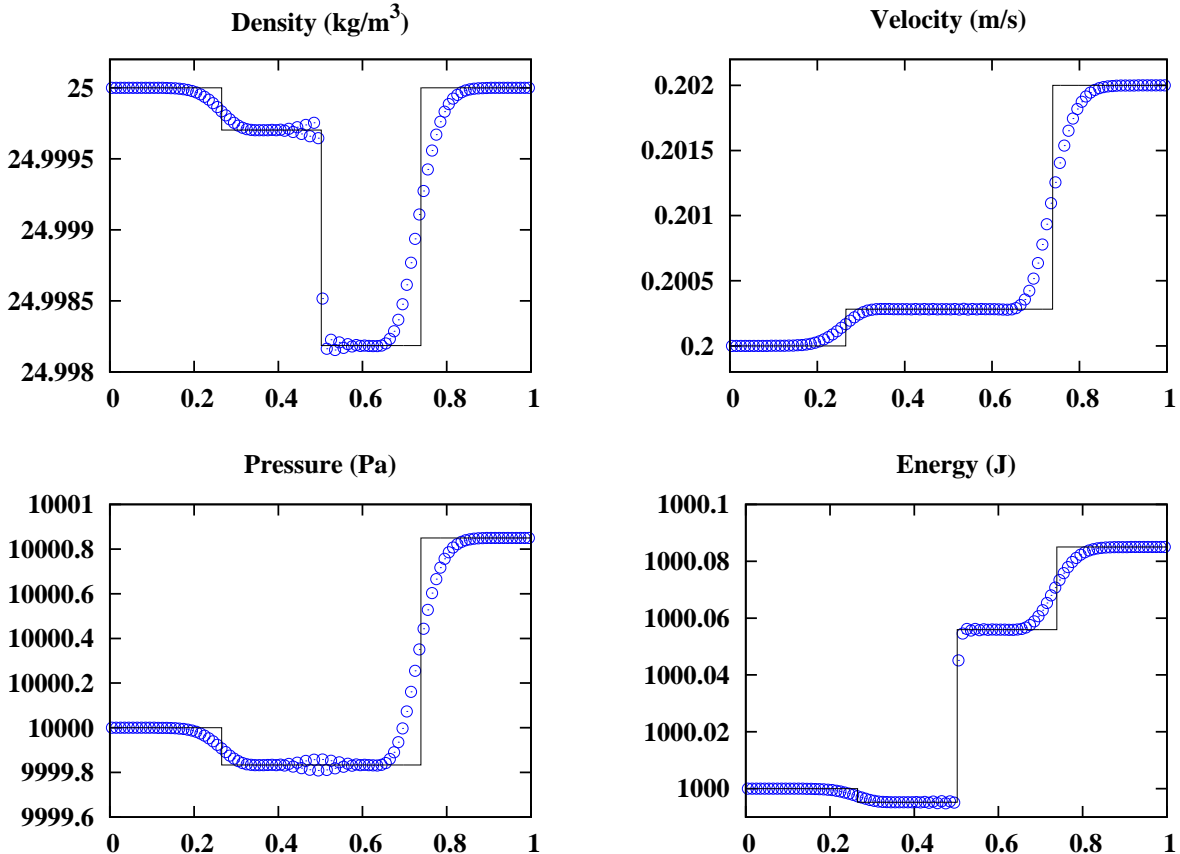

Fig. 3. Low Mach number Riemann problem of Sec. 5.1.1. AUSM ${ }^{+}$scheme (o) and exact (solid line) solutions at time $t=0.01 \mathrm{~s}$. 

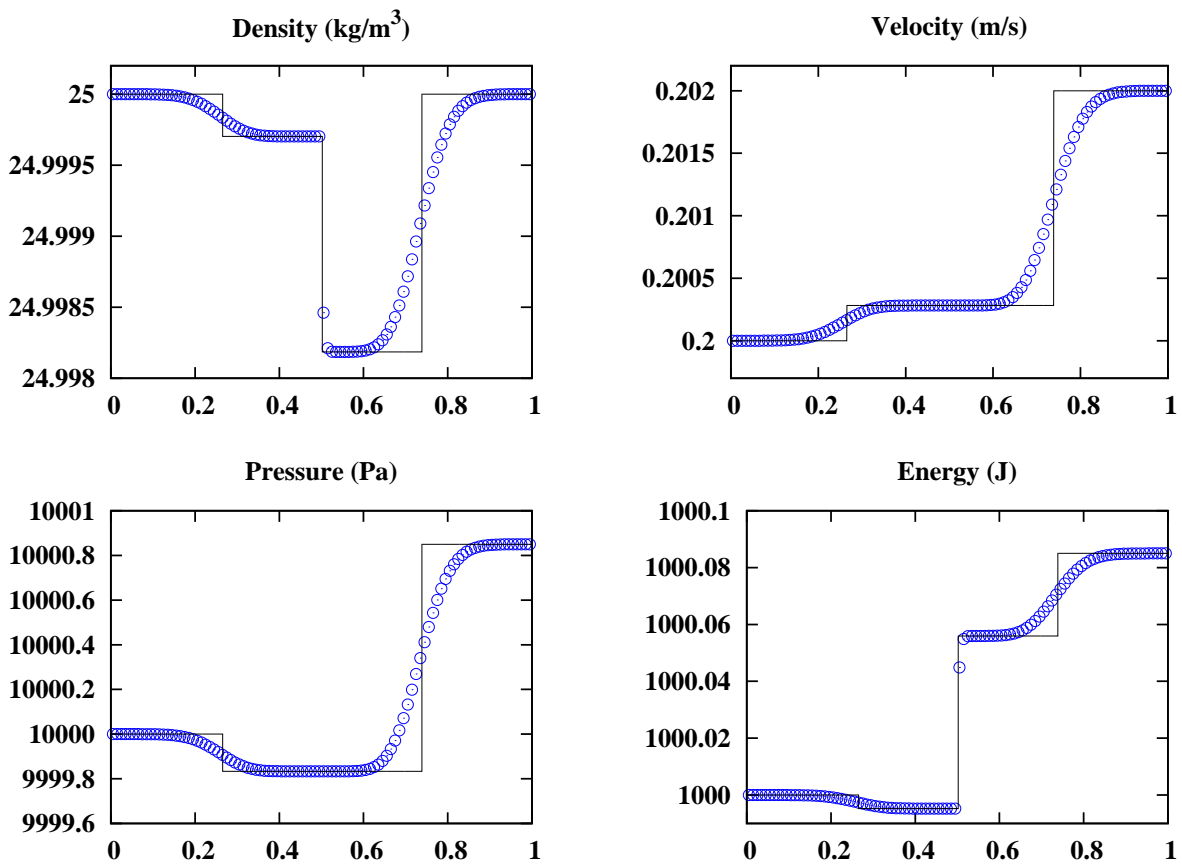

Fig. 4. Low Mach number Riemann problem of Sec. 5.1.1. AUSM $^{+}$-up scheme with $f_{c}=1$ (o) and exact (solid line) solutions at time $t=0.01 \mathrm{~s}$.
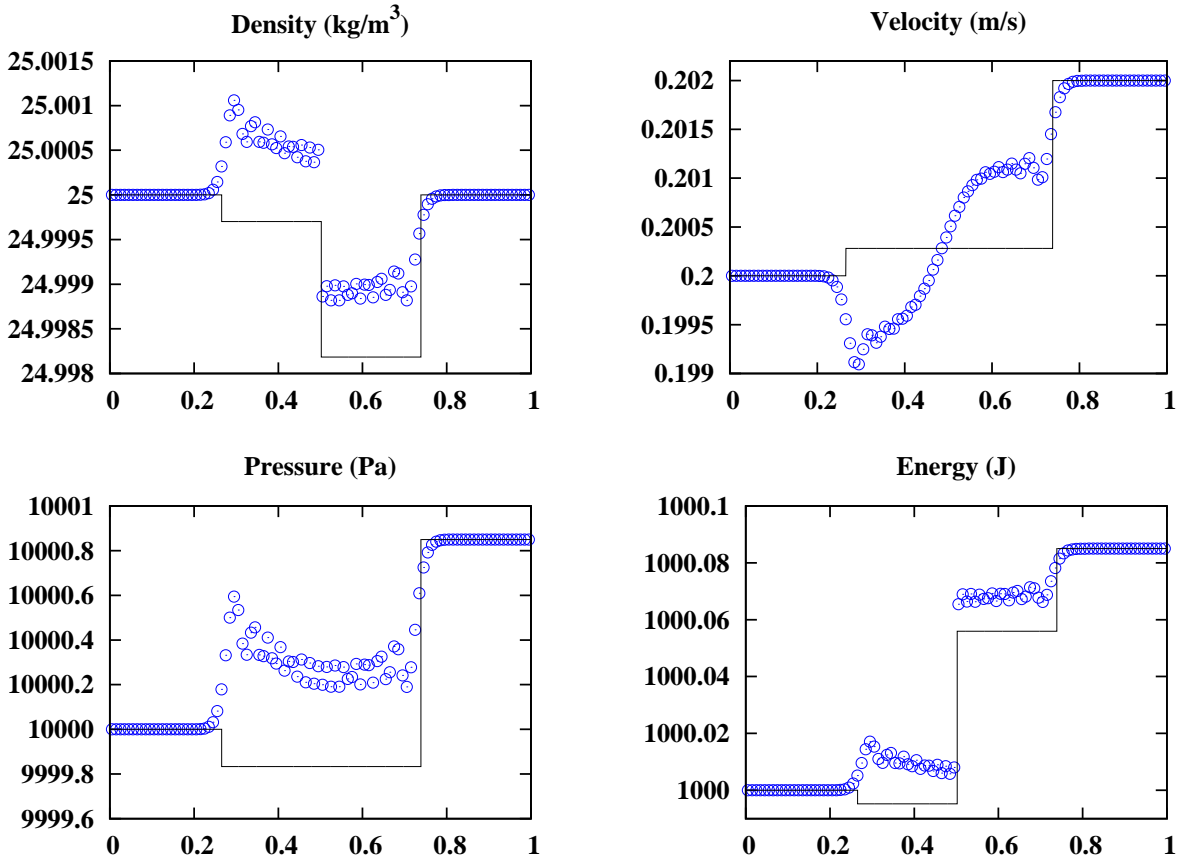

Fig. 5. Low Mach number Riemann problem of Sec. 5.1.1. Momentum interpolation (o) and exact (solid line) solutions at time $t=0.01 \mathrm{~s}$. 


\subsubsection{Downstream propagation of an acoustic wave forced at the boundary of a 1-D pipe}

At the left side of a one-meter long pipe, the inlet velocity is specified to oscillate around a mean value $V$, as

$$
v^{\dagger}(t)=V[1+A \sin (2 \pi f t)]
$$

where $V=0.30886 \mathrm{~m} / \mathrm{s}, f=210^{3} \mathrm{~Hz}$ and $A=10^{-2}$. A downstream propagating acoustic wave is thus generated and superimposed onto a mean flow with constant density $1.2046 \mathrm{~kg} / \mathrm{m}^{3}$, velocity $V=0.30886 \mathrm{~m} / \mathrm{s}$ and pressure $101300 \mathrm{~Pa}$. The amplitude $A$ is sufficiently small so that an exact solution can be derived from linear acoustics.

The Mach number of the background flow is $10^{-3}$. For the calculation of acoustic waves propagation in such a low Mach number flow, we showed in $[14,15]$ that the pressure correction algorithm presented in Appendix B is well-suited. Therefore, this algorithm is used for the present test case. The convective CFL number is chosen as $\mathrm{CFL}_{v}=5 \times 10^{-4}$, the acoustic CFL number being thus approximately equal to 0.5 . Acoustic waves can then be calculated with accuracy, on a uniform grid of 500 cells.

To allow for the assessment of the applicability and significance of the numerical results, non-dimensional length $x^{*}$ and duration $t^{*}$ are introduced. The reference length is defined as the wavelength of the acoustic wave generated at the left of the computational domain, and the reference duration is defined as the time needed for an acoustic wave to travel over the computational domain.

In Fig. 6-(a) is shown the progressive degradation of the solution obtained at time $t^{*}=0.7$ when the $\mathrm{AUSM}^{+}$-up scheme is used. The same observation holds for the SLAU scheme, to a lesser extent however (see Fig. 6-(b)). In contrast, one observes in Fig. 7 that results by the 'JCAM 2013' scheme (Fig. 7-(a)), the momentum interpolation (Fig. 7-(b)) and the AUSM-IT scheme (Fig. 7-(c)), with $K_{p}=K_{I}=0.25$ and $\sigma=1$, are in good agreement with the exact solution, and are quasi-identical.

The visible difference between the schemes for which pressure-velocity coupling is time-step dependent ('JCAM 2013', momentum interpolation and AUSM-IT) and the other schemes (AUSM ${ }^{+}$-up and SLAU) is confirmed by the dissipation and dispersion errors for the pressure field, shown in Figs. 8 and 9. Only the AUSM-IT scheme and the momentum interpolation give good results, which are very close to each other. In contrast, as already noted, results by the $\mathrm{AUSM}^{+}$-up scheme are of bad quality from the early stage of the calculation, both for dissipation and dispersion. The SLAU scheme produces results that mainly exhibit an important level of dissipation error, while the dispersion error remains at an acceptable level. This is also the case for the dispersion error obtained by the 'JCAM 2013' scheme. It is interesting to note in Fig. 8 that the dissipation error by the 'JCAM 2013' scheme 
follows the dissipation errors by the AUSM-IT scheme and by the momentum interpolation in the early stage of the calculation. The final level of the dissipation error by the 'JCAM 2013' scheme is almost two orders of magnitude lower than that of the SLAU scheme.

The sensitivity of the total acoustic energy to the cell size of the grid is shown in Fig. 10. The convective CFL number, the frequency of the acoustic wave and the Mach number of the background flow are the same as in the previous calculations, and results are shown at $t^{*}=0.7$ in order to avoid effects of wave reflection. In Fig. 10 , the total acoustic energy is non-dimensionalized by its exact analytical value at $t^{*}=0.7$. According to the theoretical considerations in Sec. 3.3, the presence of an inertia term allows to weaken the cell size dependence on the acoustic energy time evolution, in particular for the smallest values of the cell size. Indeed, this can be observed in Fig. 10 for the AUSM-IT and the momentum interpolation schemes. Note that both schemes give quasi-identical values of the total acoustic energy, which are distinctly higher than the values obtained with the other schemes. 
(a)

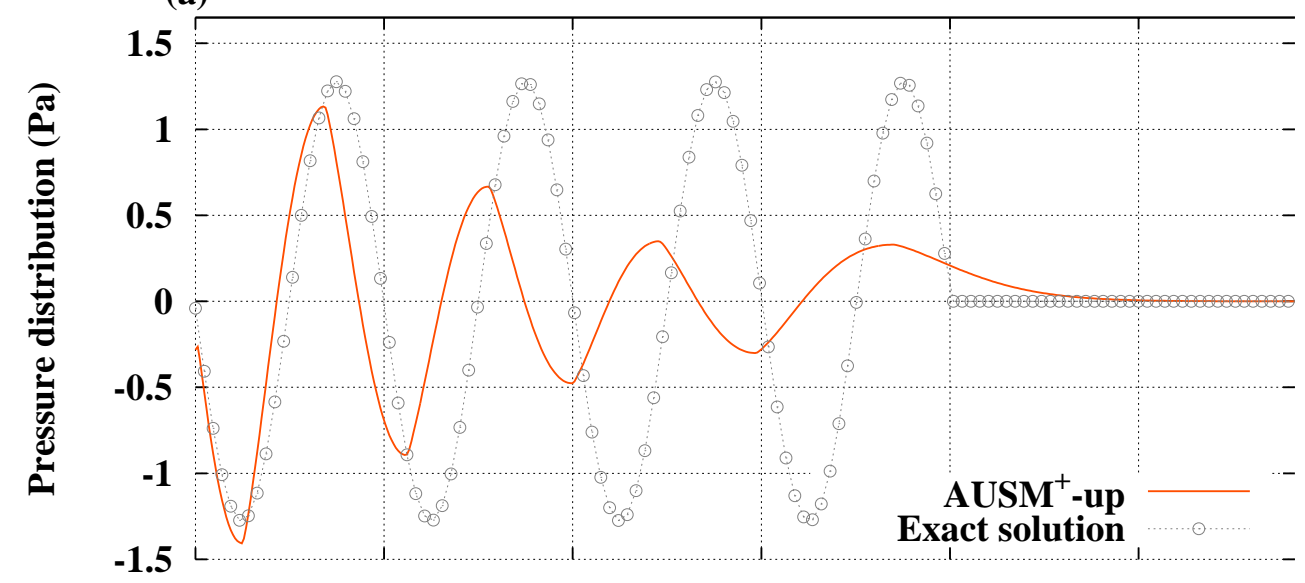

(b)

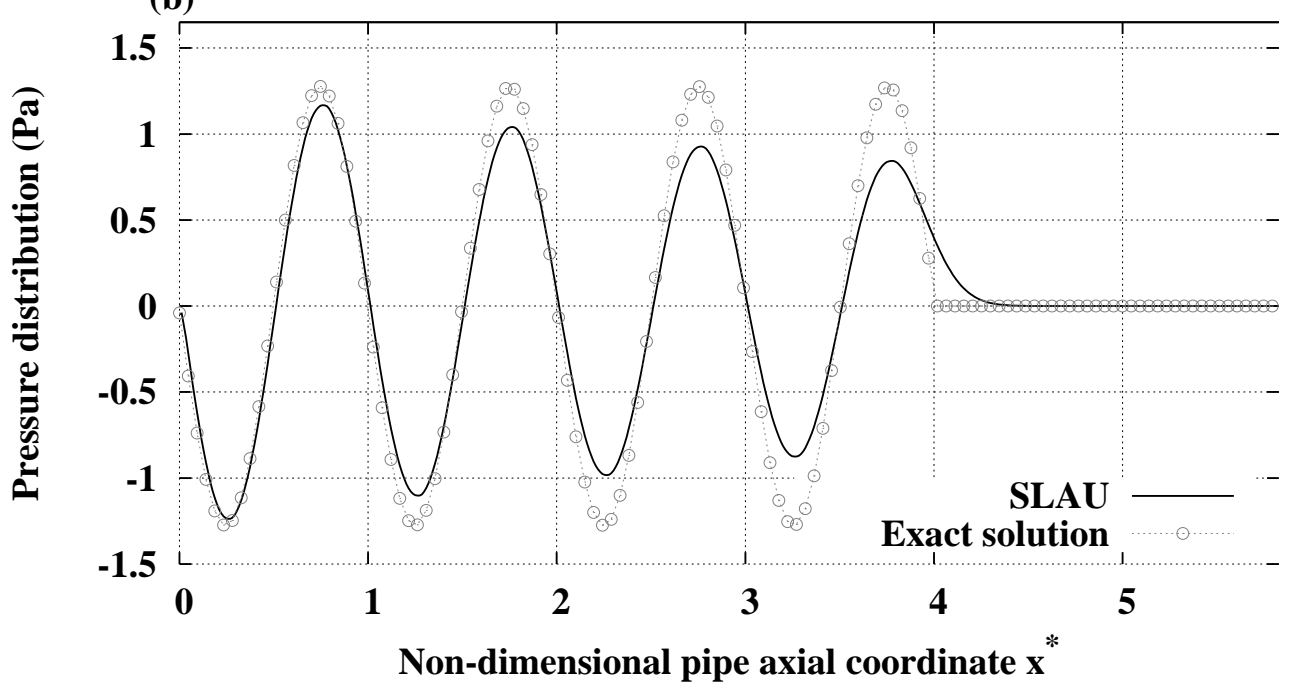

Fig. 6. Downstream propagation of a wave forced at the left boundary of a 1-D pipe, $c f$. Sec. 5.1.2. Pressure distribution at $t^{*}=0.7$. Exact solution: linear acoustics. 

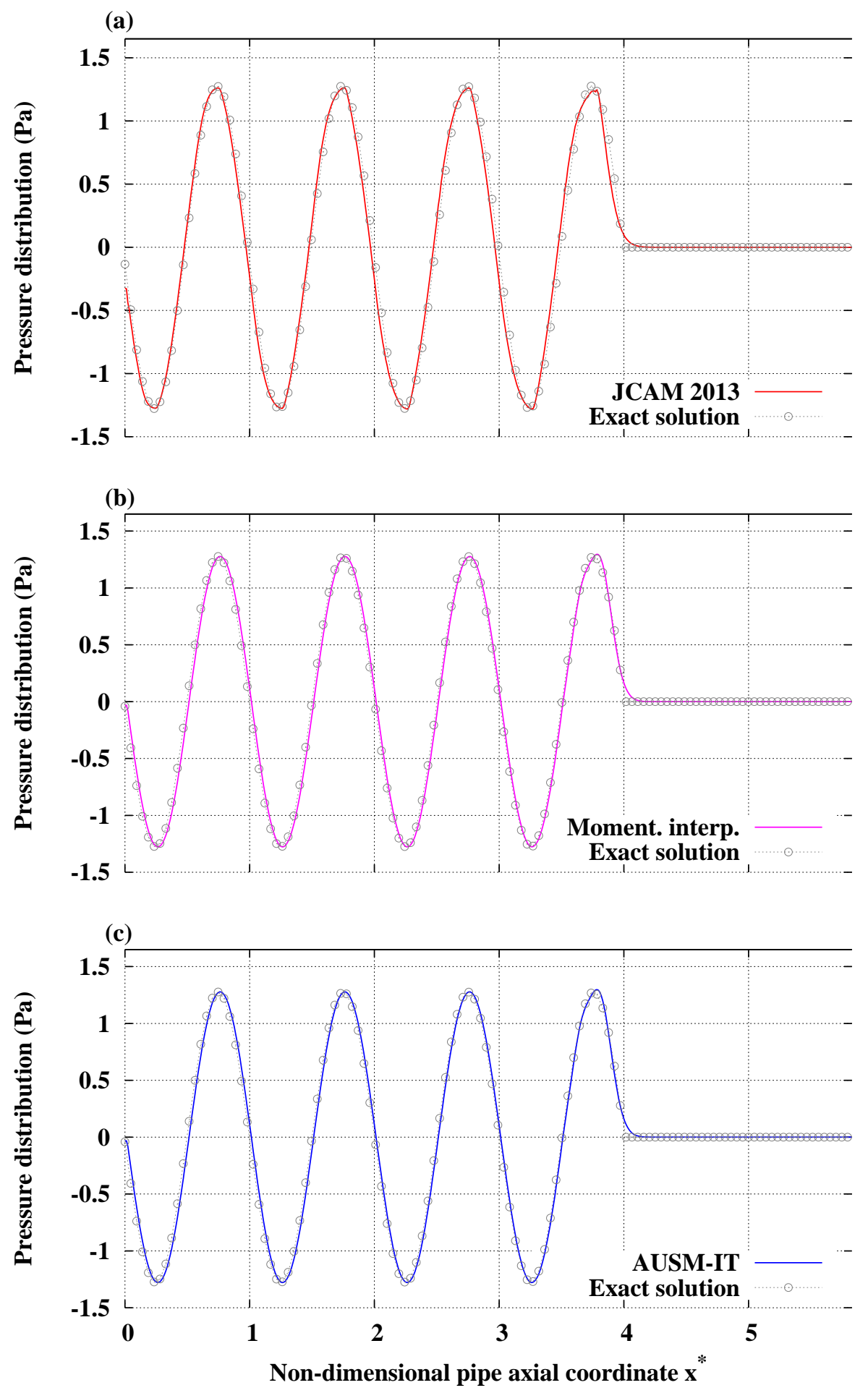

Fig. 7. Downstream propagation of a wave forced at the left boundary of a 1-D pipe, $c f$. Sec. 5.1.2. Pressure distribution at $t^{*}=0.7$. Exact solution: linear acoustics. 


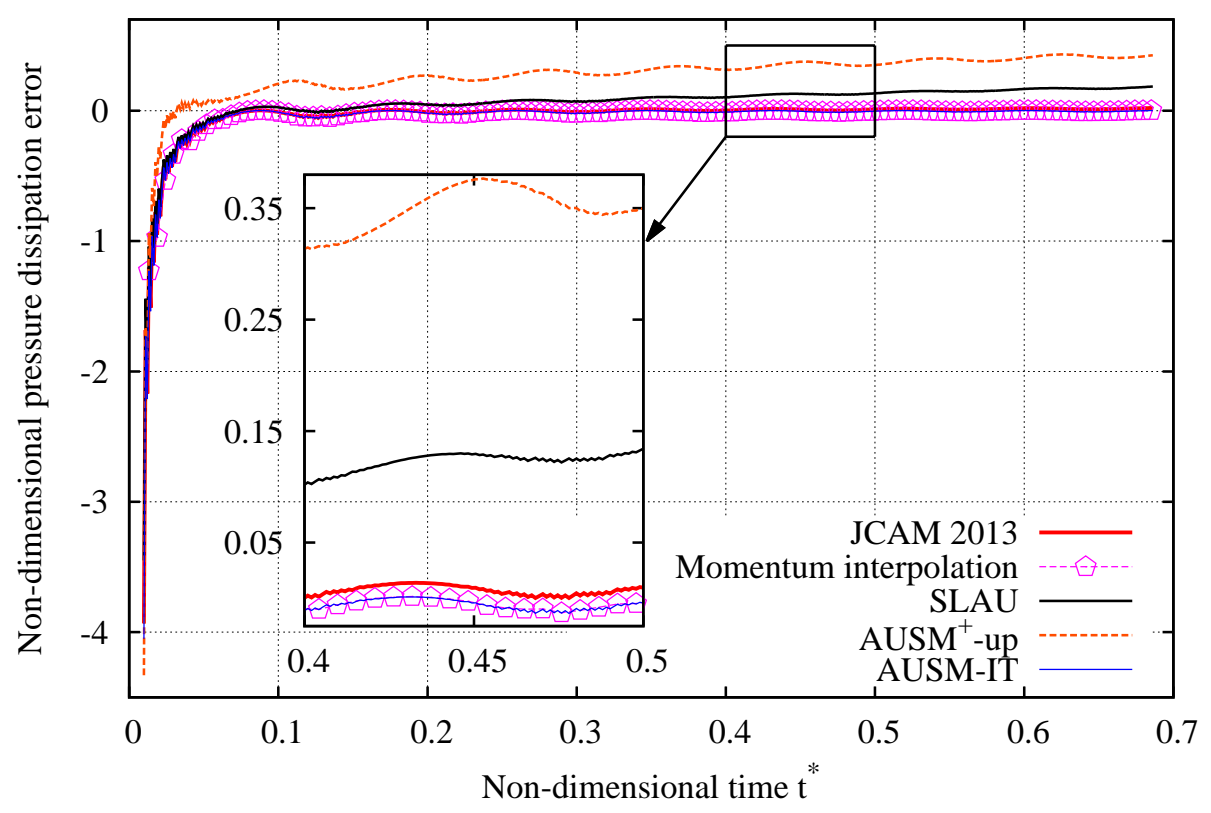

Fig. 8. Downstream propagation of a wave forced at the left boundary of a 1-D pipe, $c f$. Sec. 5.1.2. Non-dimensional time evolution of the non-dimensional dissipation error on pressure perturbation, $c f$. Eqs. (19).

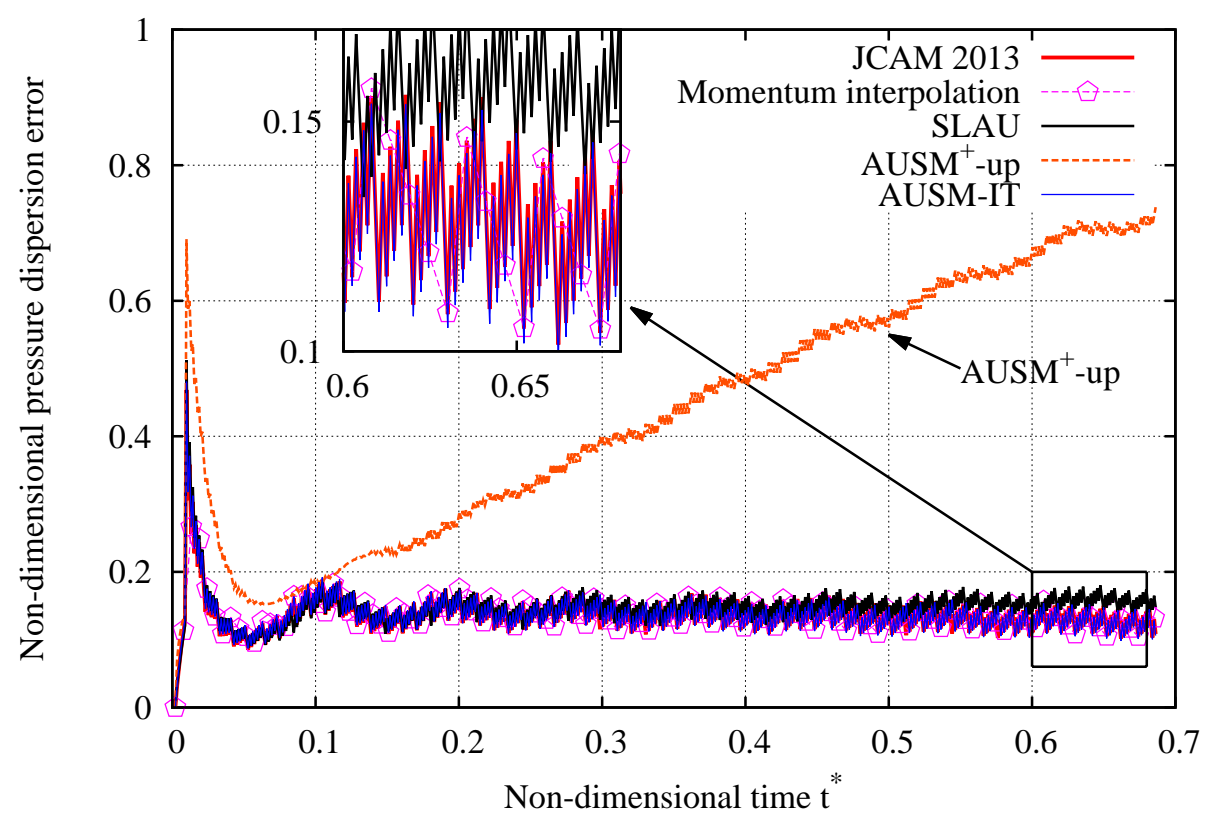

Fig. 9. Downstream propagation of a wave forced at the left boundary of a 1-D pipe, $c f$. Sec. 5.1.2. Non-dimensional time evolution of the non-dimensional dispersion error on pressure perturbation, $c f$. Eqs. (19). Results by the JCAM 2013 scheme and the AUSM-IT scheme almost coincide. 


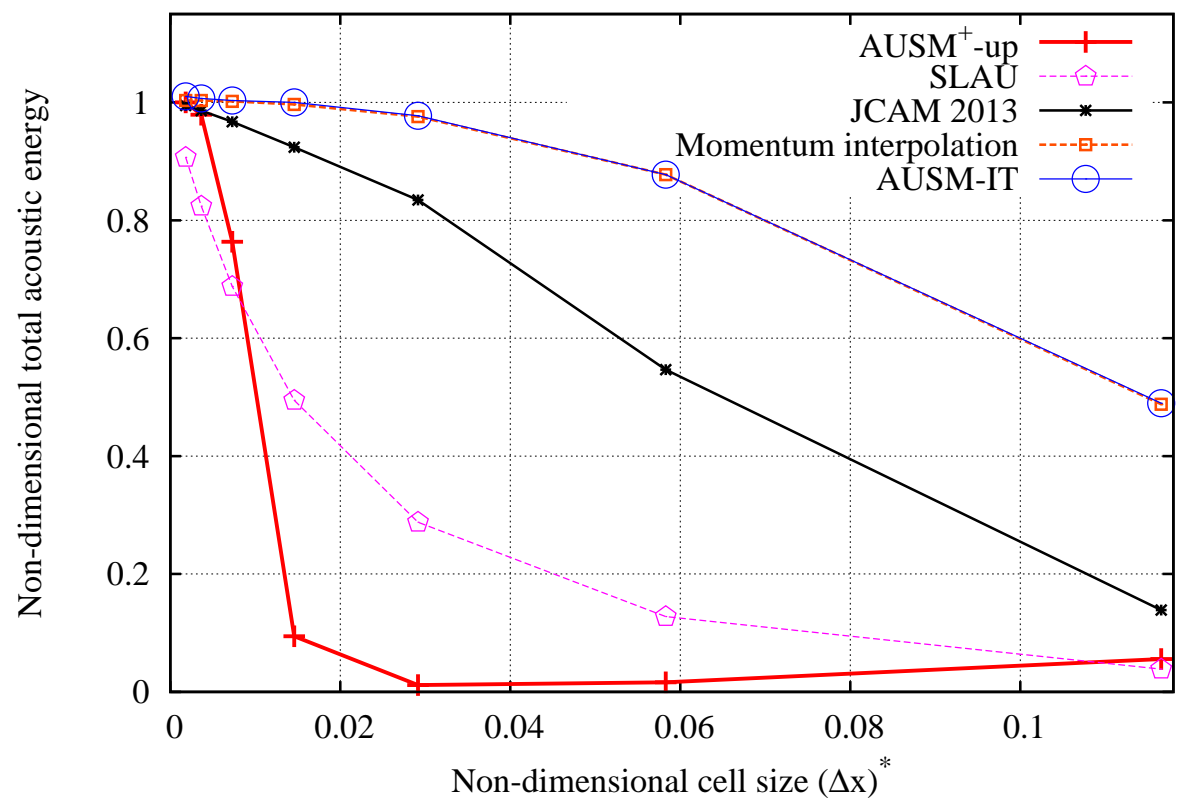

Fig. 10. Downstream propagation of a wave forced at the left boundary of a 1-D pipe, $c f$. Sec. 5.1.2. Non-dimensional total acoustic energy ( $c f$. Eq. (20)) at $t^{*}=0.7 v s$. non-dimensional cell size of the grid. Exact value of the non-dimensional total acoustic energy: 1. 


\subsubsection{One-dimensional acoustic pulse propagation}

A downstream propagating Gaussian acoustic pulse in a five-meter long pipe is generated through a superimposition onto a mean flow, with constant density $\varrho_{0}=$ $1.2046 \mathrm{~kg} / \mathrm{m}^{3}$, velocity $v_{0}=0.030886 \mathrm{~m} / \mathrm{s}$ and pressure $p_{0}=101300 \mathrm{~Pa}$, of a perturbation of pressure $\delta p$, density $\delta \varrho=\delta p / c_{0}^{2}$, and velocity $\delta v=\delta p /\left(\varrho_{0} c_{0}\right)$, where $c_{0}=\sqrt{\gamma p_{0} / \varrho_{0}}$. At $t=0$,

$$
\delta p=200 \exp \left[-\frac{(x-0.2)^{2}}{2 \sigma^{2}}\right](\mathrm{Pa}), \text { where } \sigma=210^{-2} \mathrm{~m}
$$

In this test case, the Mach number of the background flow is $10^{-4}$. The time-step is chosen so that the acoustic CFL number is about 5 , which is allowed by the semiimplicit algorithm used, see Appendix B. The grid is uniform with 2500 cells.

The results are shown with a non-dimensional length $x^{+}$, obtained by taking $\sigma$ as reference length (see Eq. (21)), and a non-dimensional duration $t^{+}$, with as reference the time needed for an acoustic wave to travel over the computational domain.

It is shown in Fig. 11 that the $\mathrm{AUSM}^{+}$-up scheme and the SLAU scheme do not reproduce correctly the propagation of the pulse ${ }^{4}$. For both schemes, the level of dissipation is excessive. The location of the pulse is erroneous with the $\mathrm{AUSM}^{+}$-up scheme. It is much better with the SLAU scheme. The 'JCAM 2013' scheme, the momentum interpolation scheme and the AUSM-IT scheme (with $K_{p}=K_{I}=0.25$ and $\sigma=1$ ) reproduce the correct position of the pulse (see Fig. 12). The dispersion error of the momentum interpolation scheme and the AUSM-IT scheme is small.

The previous observations are confirmed by studying dissipation and dispersion errors for the pressure field. Two categories of schemes can be distinguished: In the first category (AUSM ${ }^{+}$-up and SLAU schemes), the time-step independence of the pressure-velocity coupling leads rapidly to unsatisfactory levels of dissipation and dispersion errors (not shown). In the second category ('JCAM 2013', momentum interpolation and AUSM-IT), the unsteadiness of the calculated waves is taken into account in the pressure-velocity coupling, thanks to the time-step in the pressure

4 The small difference between the results shown in Fig. 11 (a) and the results shown in Fig. 3 of our previous work [14], where the same test case is considered with the AUSM ${ }^{+}$up scheme too, is due to the choice of the interpolation of the pressure corrections; see Eq. (31). In [14], interpolation polynomials are defined with the scaling function $f_{c}$ of the $\mathrm{AUSM}^{+}$-up scheme proposed in [11]. Here ( $c f$. Eq. (31)), interpolation polynomials of the $\mathrm{AUSM}^{+}$scheme, without the scaling function $f_{c}$, are used for the pressure corrections. This results for the present test case in a smaller dissipation than in the computations shown in [14]. The modification of the definition of the interpolation polynomials also has as a consequence to reduce the dispersion error for the 'JCAM 2013' scheme (cf. Fig. 12 (c)), if compared to results shown in Fig. 3 of [14]. 
gradient coefficient of the face velocity expression. As shown in Figs. 13 and 14, this time-step dependence is necessary for obtaining accuracy, for both dissipation and dispersion features. Moreover, it is clear, in particular from Fig. 13, that, due to the presence of inertia term in the face velocity expression of the AUSM-IT and the momentum interpolation schemes, both schemes outperform the 'JCAM 2013' scheme, for which the true inertia term is not included.

The same conclusion holds by considering the time evolution of the total acoustic energy (see Eq. (20)). Presented in Fig. 15, the total acoustic energy is nondimensionalized with its exact value at $t^{+}=0$. Note that results by the $\mathrm{AUSM}^{+}$-up and SLAU schemes are useless (not shown). In Fig. 15, the levels of total acoustic energy given by the 'JCAM 2013' scheme, the AUSM-IT scheme and the momentum interpolation, are identical in the early stage of the calculation. The calculated value is slightly lower than the exact value, which is constant and equal to 1 . Among the three schemes under consideration, the conservation of the acoustic energy in the computational domain is best ensured by the momentum interpolation. With the AUSM-IT scheme, the energy increases slightly (by less than 1\%), and with the 'JCAM 2013' scheme, it decreases by about $18 \%$.

The sensitivity of the total acoustic energy to the cell size of the grid is shown in Fig. 16. The convective CFL number, the frequencies of the acoustic waves and the Mach number of the background flow are the same as in the previous calculations. Results are shown at $t^{+}=0.8$ in order to avoid effects by wave reflection. The total acoustic energy is non-dimensionalized by its exact analytical value at $t^{+}=0$. As already observed in Sec. 5.1.2, the presence of an inertia term allows to weaken the cell size dependence of the acoustic energy time evolution. When the AUSM-IT scheme is used, the level of the total acoustic energy for the smallest values of $(\Delta x)^{+}$in Fig. 16 is slightly higher than 1 . The level of dissipation of the scheme does not guarantee exactly the conservation of the total acoustic energy. The level of dissipation of the AUSM-IT scheme is linked with the upwinding of the convective term $c_{1 / 2} M_{1 / 2}$ in the face velocity expression (16). This upwinding is a consequence of the choice of the $\mathrm{AUSM}^{+}$polynomials in the definition of $M_{1 / 2}$, which were not designed to ensure the property of the total energy conservation. However, this property is approximately satisfied, which is quite satisfactory by comparison with the other schemes under consideration. 


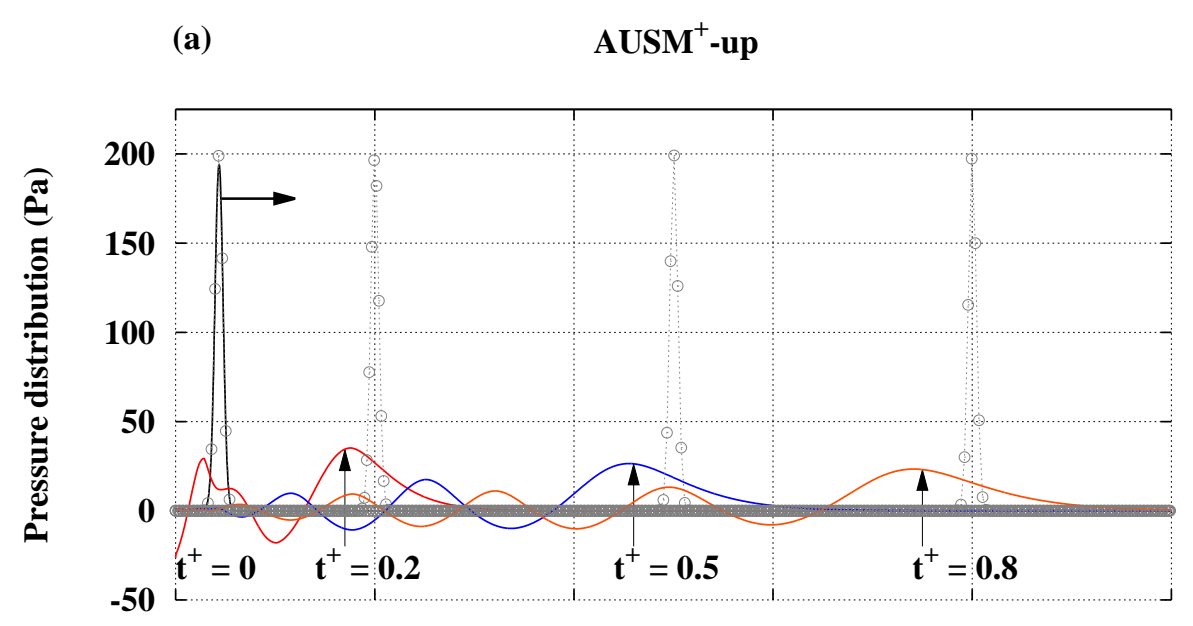

(b)

SLAU

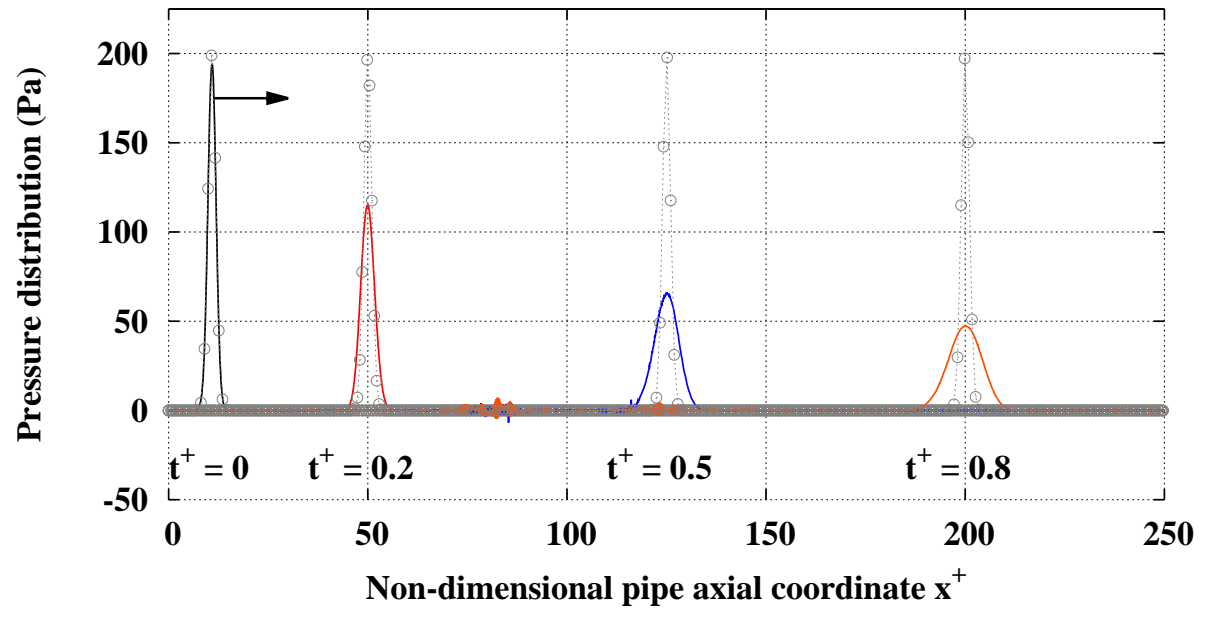

Fig. 11. Downstream propagation of an acoustic pulse in a five-meter long pipe, $c f$. Sec. 5.1.3. Pressure distribution at $t^{+}=0, t^{+}=0.2, t^{+}=0.5$ and $t^{+}=0.8$. Exact solution (linear acoustics): $-\circ-$. 
(a)

JCAM 2013

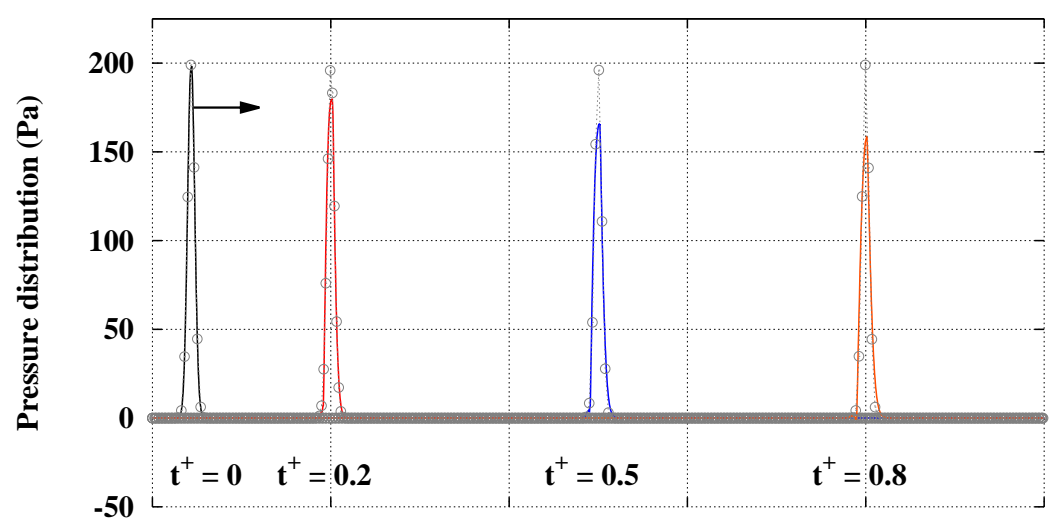

(b)

Momentum interpolation

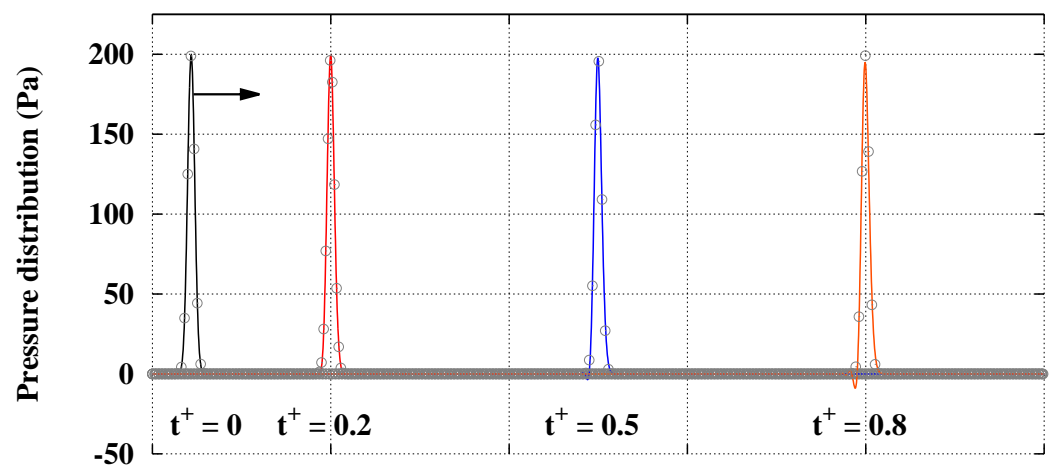
(c)
AUSM-IT

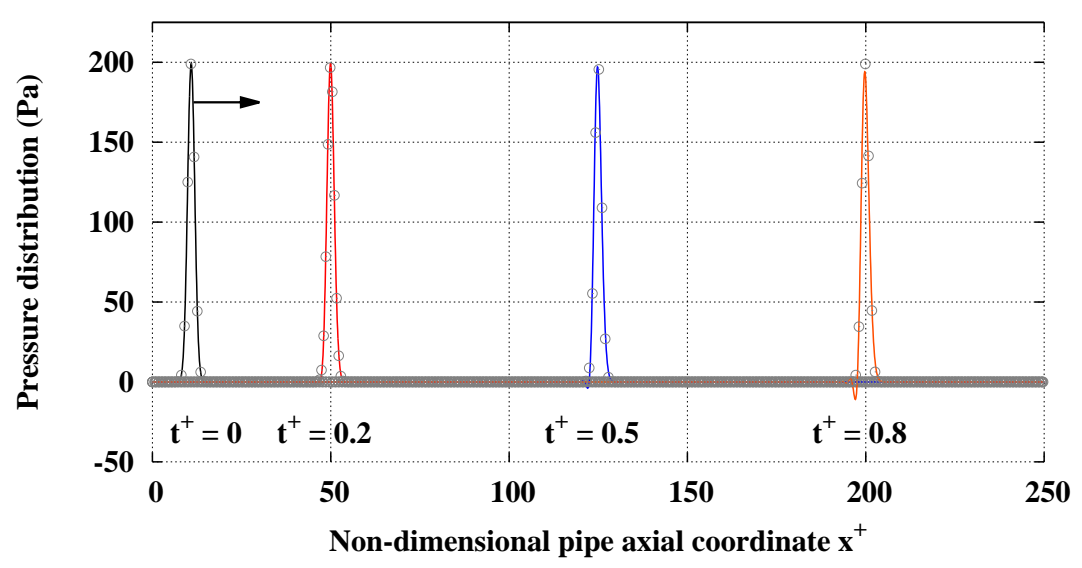

Fig. 12. Downstream propagation of an acoustic pulse in a five-meter long pipe, $c f$. Sec. 5.1.3. Pressure distribution at $t^{+}=0, t^{+}=0.2, t^{+}=0.5$ and $t^{+}=0.8$. Exact solution (linear acoustics): $-\circ-$. 


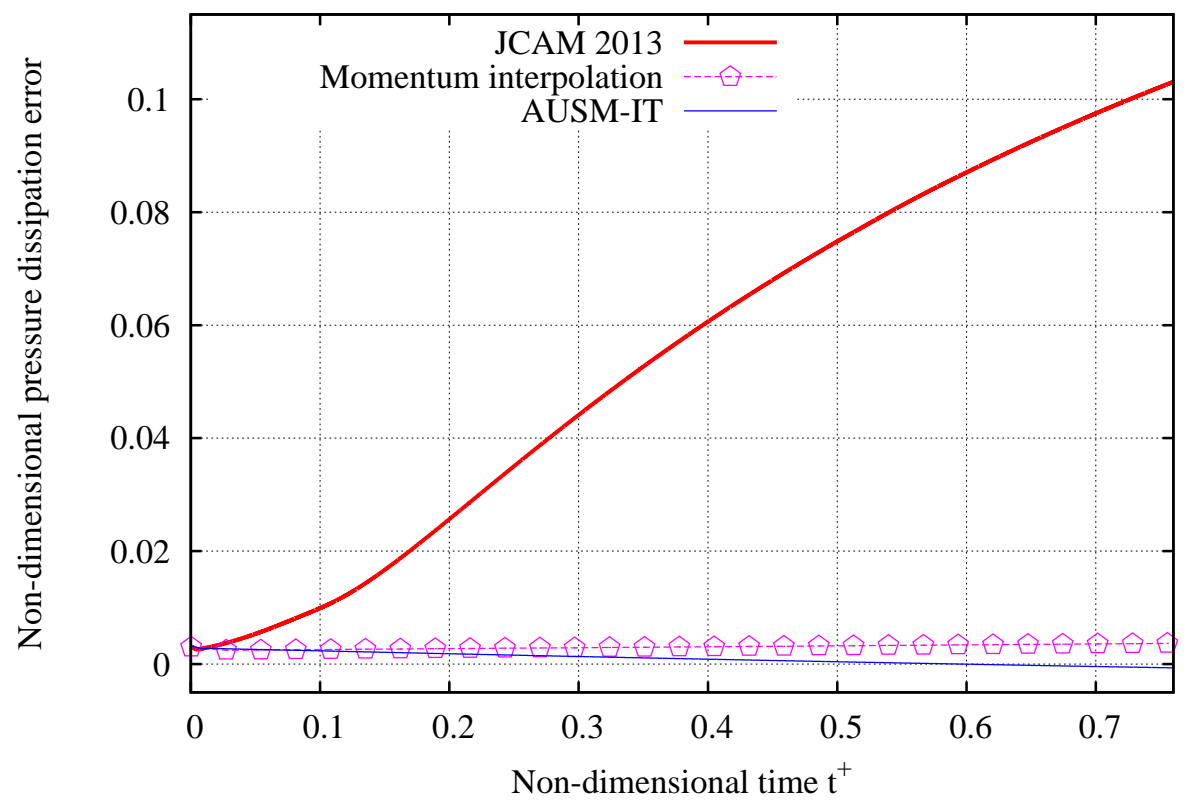

Fig. 13. Downstream propagation of an acoustic pulse in a five-meter long pipe, $c f$. Sec. 5.1.3. Non-dimensional time evolution of the non-dimensional dissipation error on pressure perturbation, $c f$. Eqs. (19).

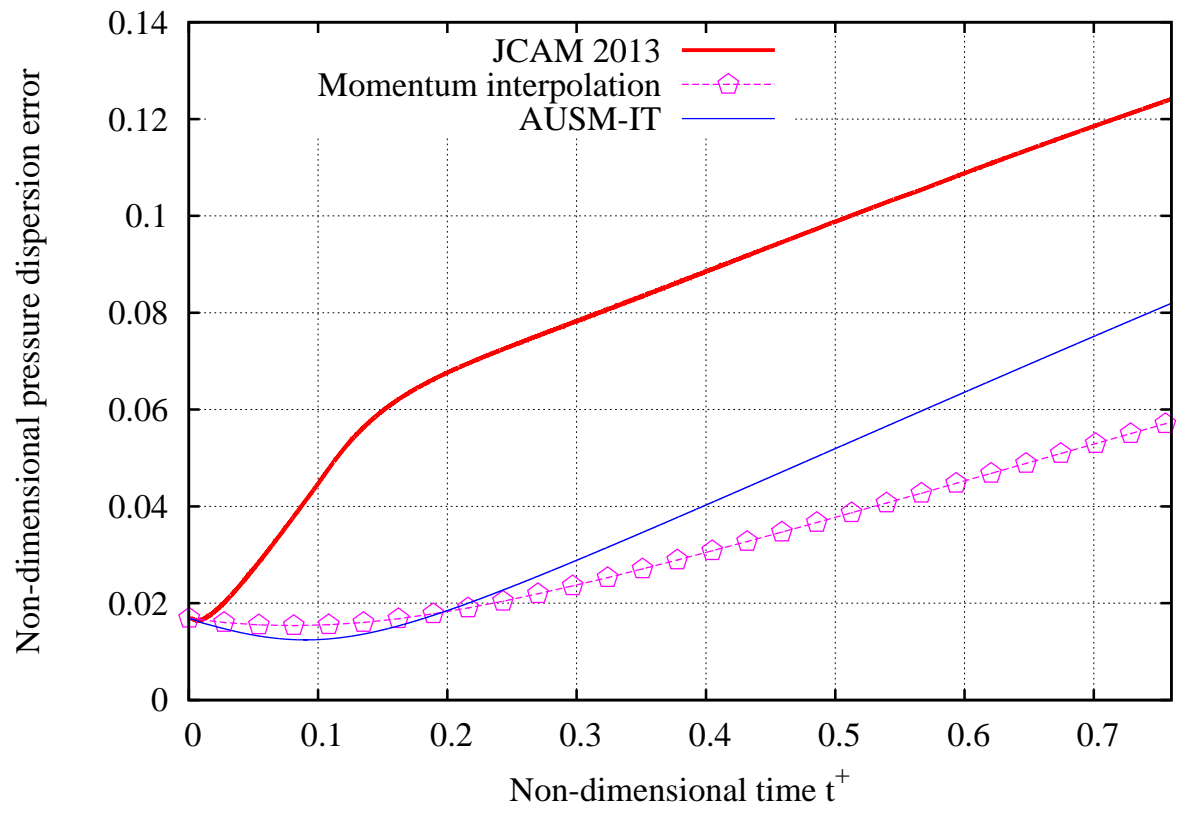

Fig. 14. Downstream propagation of an acoustic pulse in a five-meter long pipe, $c f$. Sec. 5.1.3. Non-dimensional time evolution of the non-dimensional dispersion error on pressure perturbation, $c f$. Eqs. (19). 


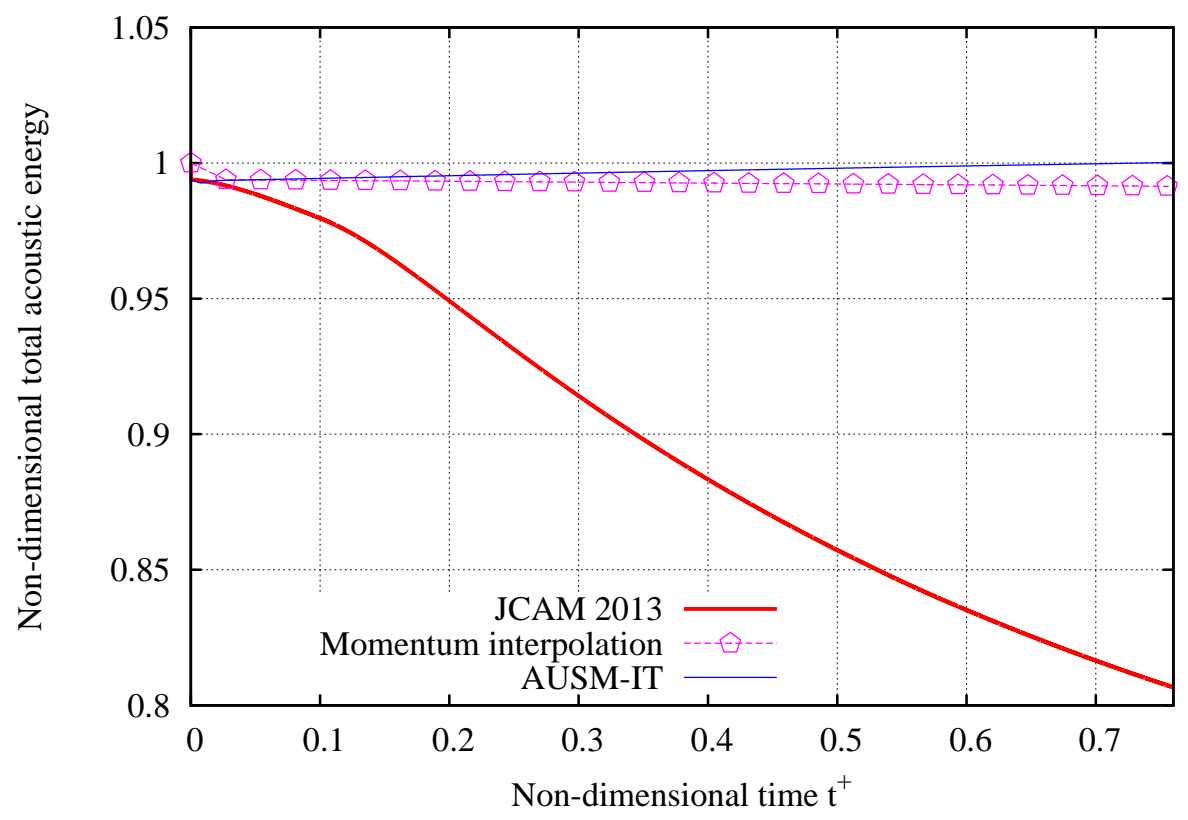

Fig. 15. Downstream propagation of an acoustic pulse in a five-meter long pipe, $c f$. Sec. 5.1.3. Non-dimensional time evolution of the non-dimensional total acoustic energy, $c f$. Eq. (20) (exact value of the non-dimensional total acoustic energy: 1).

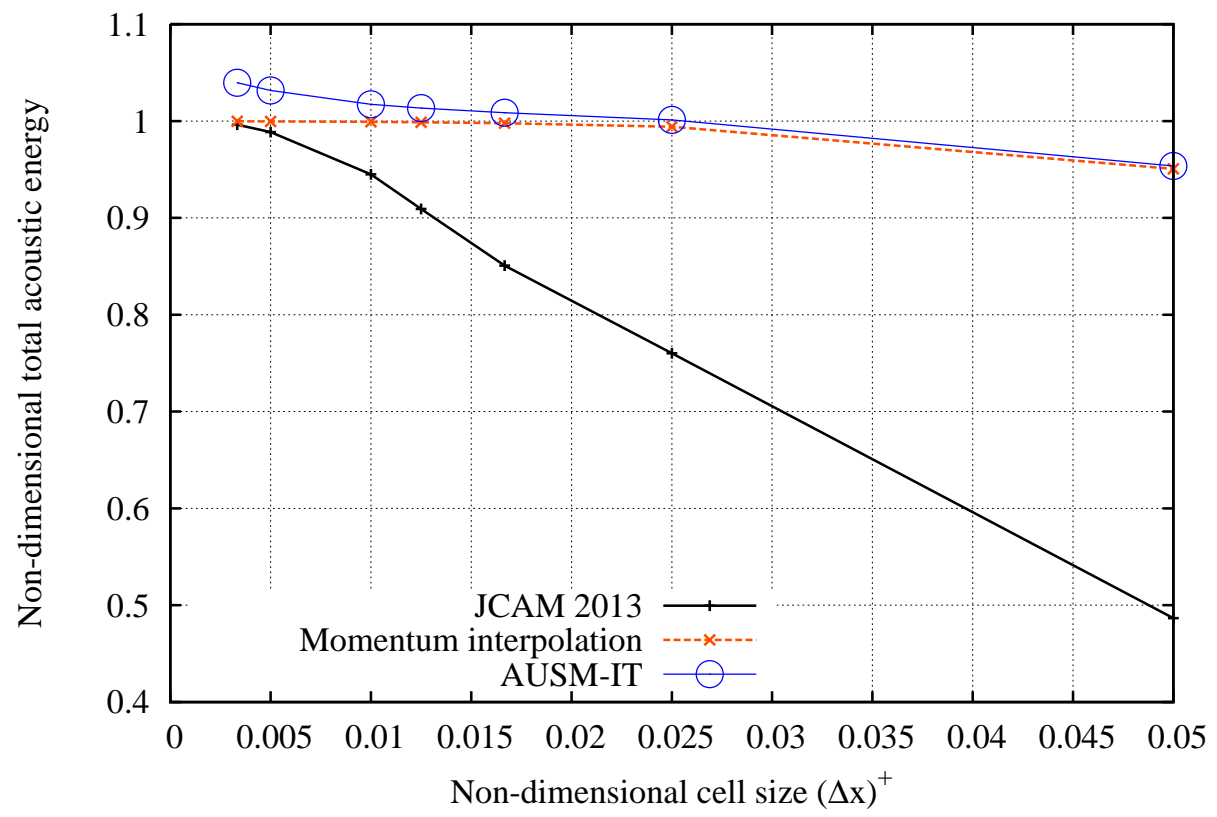

Fig. 16. Downstream propagation of an acoustic pulse in a five-meter long pipe, $c f$. Sec. 5.1.3. Non-dimensional total acoustic energy ( $c f$. Eq. (20)) at $t^{+}=0.8 v s$. non-dimensional cell size of the grid (exact value of the non-dimensional total acoustic energy: 1). 


\subsubsection{Two-dimensional acoustic pulse propagation}

The computational domain is the square $[0,1] \times[0,1]\left(\mathrm{m}^{2}\right)$, divided into $500 \times 500$ cells forming a regular Cartesian grid. With

$$
\varrho_{0}=1.2046 \mathrm{~kg} / \mathrm{m}^{3}, u_{0}=v_{0}=0.3088610^{-2} \mathrm{~m} / \mathrm{s}, p_{0}=101300 \mathrm{~Pa},
$$

a Gaussian acoustic pulse is given at $t=0$ by

$$
\varrho^{0}=\varrho_{0}+(\delta \varrho)^{0}, u^{0}=u_{0}, v^{0}=v_{0}, p^{0}=p_{0}+(\delta p)^{0},
$$

where

$$
\begin{aligned}
& (\delta p)^{0}=200 \exp \left[-\frac{(x-0.5)^{2}+(y-0.5)^{2}}{(0.05)^{2}}\right](\mathrm{Pa}), \\
& (\delta \varrho)^{0}=(\delta p)^{0} / c_{0}^{2} \text { and } c_{0}=\sqrt{\gamma p_{0} / \varrho_{0}} .
\end{aligned}
$$

The value of the mean flow Mach number is $910^{-6}$. For $t>0$, the speed of the acoustic wave generated by the initial pulse is the vector sum of the low Mach number mean flow velocity and the propagation sound speed in the radial direction.

The numerical method is the 2-D direct extension of the method detailed in Appendix B. The energy equation used in the correction step (see Eq. (30) for the 1-D case) results in a pentadiagonal system solved with an alternate direction procedure. The acoustic CFL number is 20. The AUSM-IT scheme is used with $K_{p}=$ $K_{I}=0.25$ and $\sigma=1$.

At $t=0.1 \mathrm{~ms}$, the maximum of the pressure forms a circle (see Fig. 18) whose radius is approximately $0.048 \mathrm{~m}$ (see Fig. 19). At $t=1 \mathrm{~ms}$, the radius is approximately $0.358 \mathrm{~m}$ (see Figs. 20 and 21). The radial speed of the acoustic wave is thus around $344.44 \mathrm{~m} / \mathrm{s}$. This is close to the value calculated with the background flow values, $c_{0}=\sqrt{\gamma p_{0} / \varrho_{0}}=343.12 \mathrm{~m} / \mathrm{s}$. As for the 1-D test of Gaussian pulse propagation considered in Sec. 5.1.3, results by the AUSM-IT scheme are quasi-identical to those by the momentum interpolation method (see Figs. 19 and 21). 


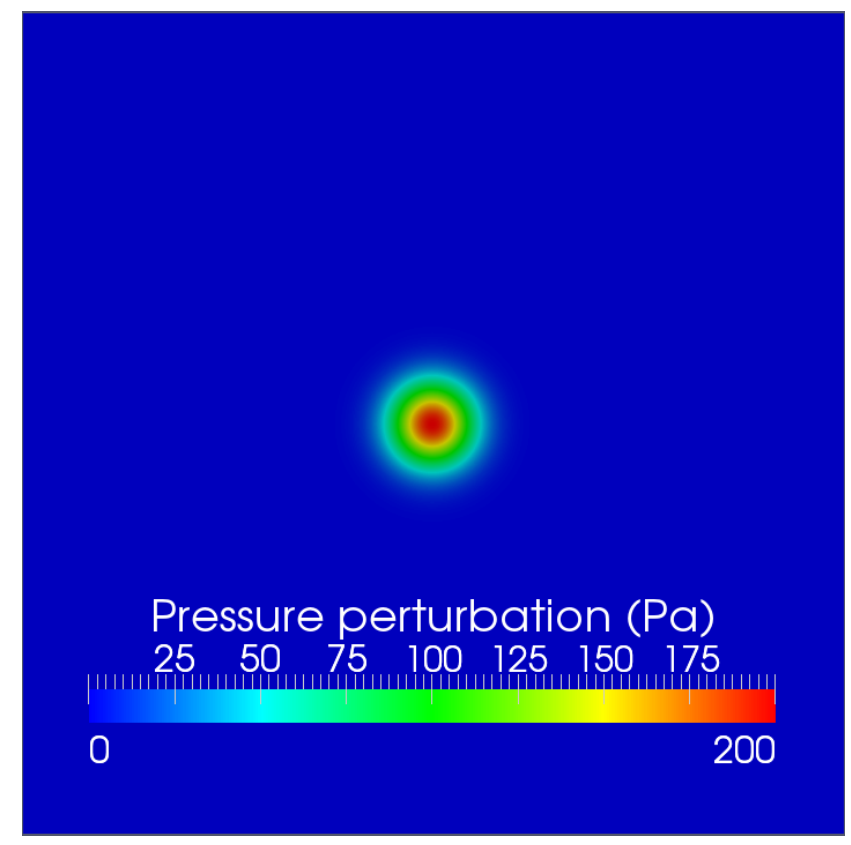

Fig. 17. Two-dimensional acoustic pulse propagation, $c f$. Sec. 5.1.4. Distribution of the pressure perturbation $\delta p$ at $t=0$ (cf. Eq. (22)).

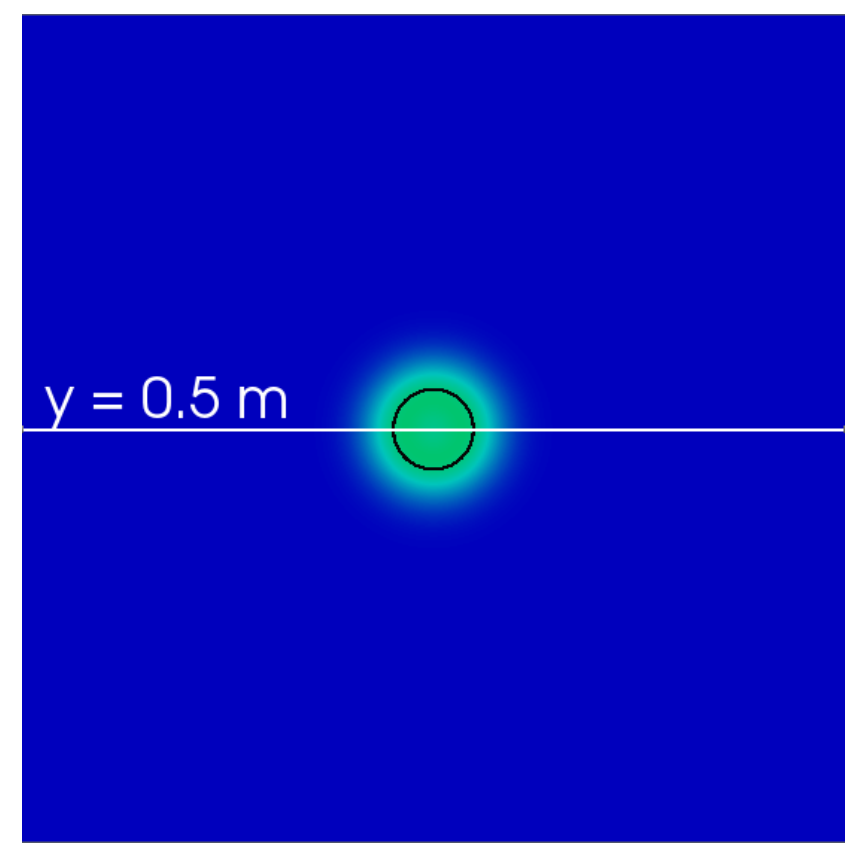

Fig. 18. Two-dimensional acoustic pulse propagation, $c f$. Sec. 5.1.4. Distribution of the pressure perturbation at $t=0.1 \mathrm{~ms}$ by the AUSM-IT scheme. The black circle indicates the maximum value of the pressure perturbation. The profile at $y=0.5 \mathrm{~m}$ (white line) is shown in Fig. 19. 


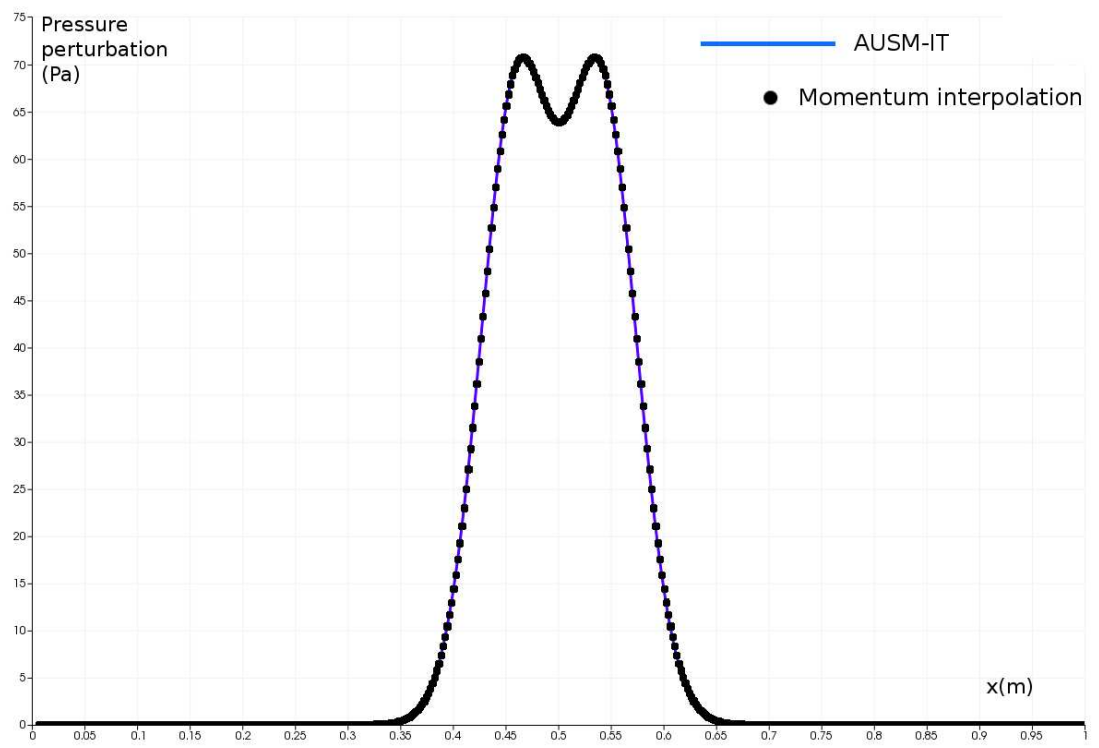

Fig. 19. Two-dimensional acoustic pulse propagation, $c f$. Sec. 5.1.4. Distribution of the pressure perturbation profiles at $t=0.1 \mathrm{~ms}$ for $y=0.5 \mathrm{~m}$ (white line in Fig. 18) by the AUSM-IT scheme (solid line) and the momentum interpolation method $(\bullet)$.

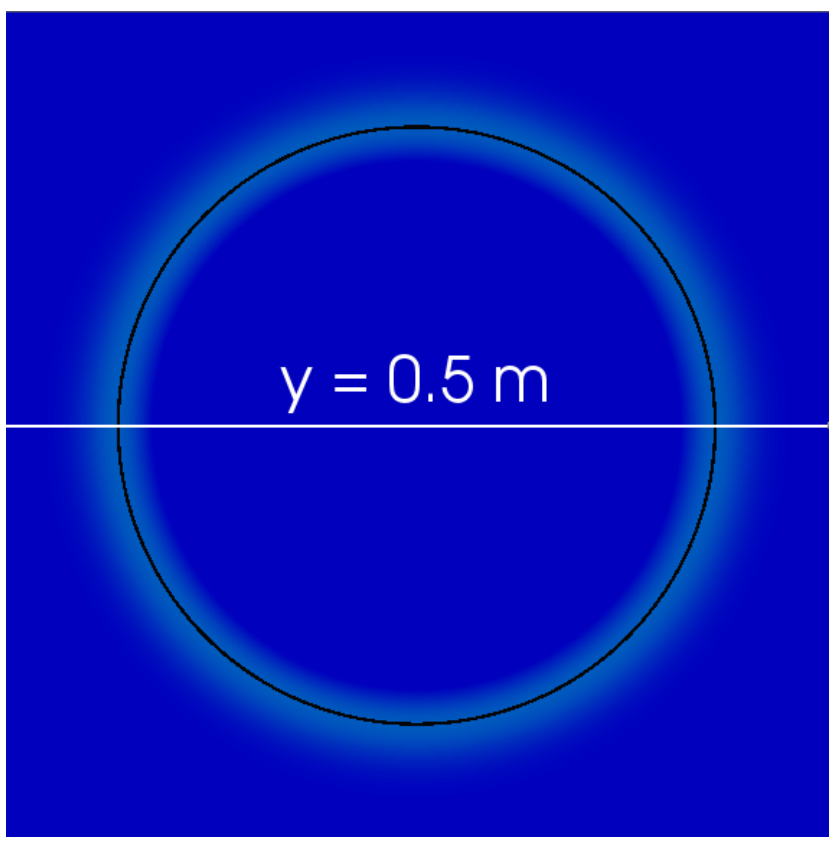

Fig. 20. Two-dimensional acoustic pulse propagation, $c f$. Sec. 5.1.4. Distribution of the pressure perturbation at $t=1 \mathrm{~ms}$ by the AUSM-IT scheme. The black circle indicates the maximum value of the pressure perturbation. The profile at $y=0.5 \mathrm{~m}$ (white line) is shown in Fig. 21. 


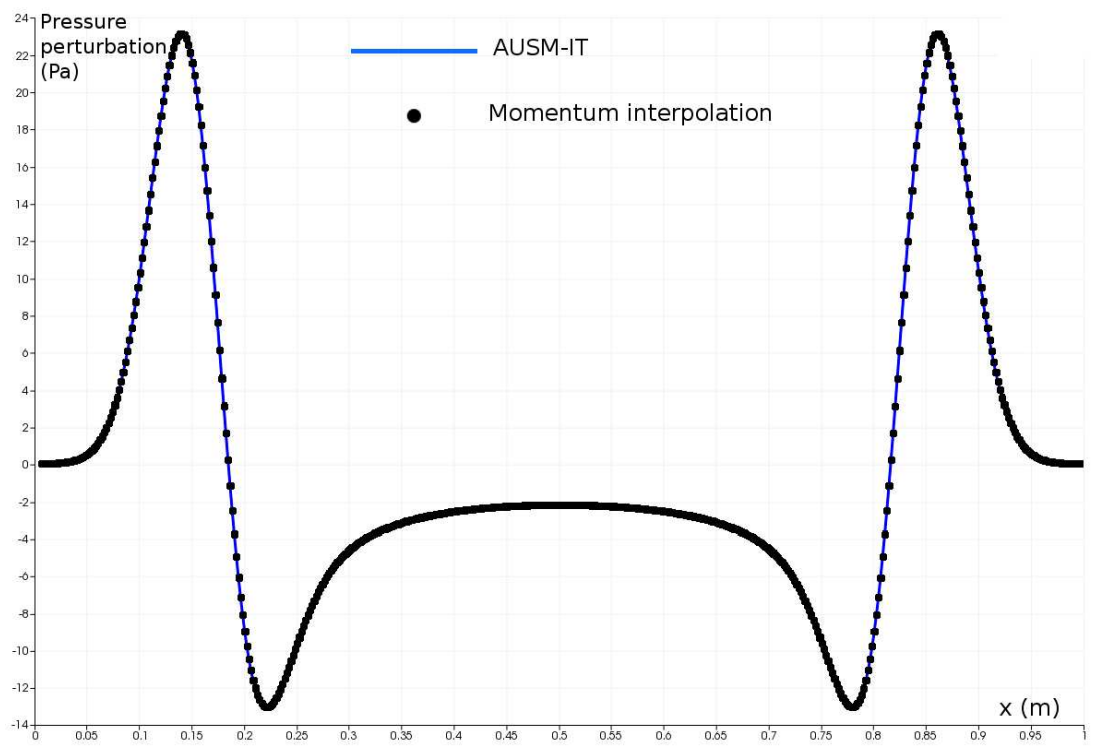

Fig. 21. Two-dimensional acoustic pulse propagation, $c f$. Sec. 5.1.4. Distribution of the pressure perturbation profiles at $t=1 \mathrm{~ms}$ for $y=0.5 \mathrm{~m}$ (white line in Fig. 20) by the AUSM-IT scheme (solid line) and the momentum interpolation method $(\bullet)$. 


\subsection{Riemann problems with shock waves}

In this section, the Godunov-type and momentum interpolation schemes are evaluated on a series of Riemann problems where shock waves occur. Moreover, the Mach number is not necessarily small compared to unity (i.e. of the order of $10^{-1}$ or smaller), as it is in Sec. 5.1.1. The settings for the three tests considered are given in Table 2. The computational domain and its discretization are the same as in Sec. 5.1.1. The Euler explicit scheme is used, with the convective CFL number equal to 0.9. The numerical method is first-order accurate in time and space. As in Sec. 5.1.1, we set $M_{\infty}=0.01$ for all the calculations presented in this section (see Appendix A), noticing as in Sec. 5.1.1 that the exact value of this parameter, if sufficiently small, does not influence significantly the results.

Test 1 is the modified Sod problem proposed in [24], to assess the fulfillment of the entropy property. The solution consists of a right sonic shock wave, a right traveling contact wave, and a left sonic rarefaction wave. Also proposed in [24], Test 2 is used to assess the robustness of the scheme, and its ability to capture slowly moving contact discontinuities. The exact solution consists of a left rarefaction wave, a right-traveling shock wave and a stationary contact discontinuity. Note that the SLAU scheme and the momentum interpolation scheme fail (i.e. do not allow convergence to a solution) in this test. Test 3, proposed in [7] (see also [18]), allows primarily to assess the accuracy of the scheme. In Test 3 , the flow remains subsonic. The solution contains a contact discontinuity and a weak shock that are close to each other, so that the contact discontinuity may be smeared by the flux scheme, in particular when it is first-order accurate, which is the case here.

We will focus the discussion of the results on the following key features: (1) shock resolution, in particular in presence of a close contact discontinuity; (2) capture of stationary contact discontinuity ; (3) sonic glitch (see [23]).

In Figs. 22 to 28, for test 1, where the right shock wave is sonic, the AUSM-type schemes give a good resolution of the shock, compared to the SLAU scheme (see Fig. 29) and the momentum interpolation (see Fig. 30). Observe that this latter leads to results of very bad quality, whatever the Riemann problem considered in the present section. Between the AUSM-type schemes, some differences can be observed in the quality of the shock representation. The $\mathrm{AUSM}^{+}$-up scheme (see Figs. 25-27) smears the shocks more than the other AUSM-type schemes. The AUSMIT scheme slightly overestimates the maximum value of the velocity at the sonic point. However, the differences between results by AUSM-type schemes remain small, and we conclude that the presence of an inertia term does not alter the ability of AUSM-IT to properly capture sonic shocks.

A sonic glitch appears in the rarefaction fan of the solution calculated by the AUSM-IT scheme, the AUSM+-up scheme and the 'JCAM 2013' scheme (see 
Figs. 22, 25-28). Note that this occurs for $K_{v}=0$ as well as for $K_{v}=0.75$ with the $\mathrm{AUSM}^{+}$-up scheme. In contrast, only a very light glitch occurs with the $\mathrm{AUSM}^{+}$scheme (see Fig. 24), using the interface sound speed of the $\mathrm{AUSM}^{+}$-up scheme (see Appendix A). We conclude that, when AUSM-type schemes are used, a sonic glitch may be formed in a sonic rarefaction fan if the pressure gradient term is present in the mass flux expression. Of course, the sonic glitch may be removed by including some numerical dissipation in the mass flux expression. Note however that no sonic glitch appears in Fig. 29, with the SLAU scheme, even if its mass flux expression contains a pressure gradient term (see Eq. (27)). This indicates that the sonic glitch does not always result from the presence of a pressure gradient term in the mass flux expression. Concerning the sonic glitch, we conclude that it is independent of the presence of an inertia term, as introduced in the AUSM-IT scheme.

For test 2 (see Figs. 31-36), conclusions are that the AUSM-type schemes perform well, provided that some pressure dissipation is included in the mass flux expression $\left(K_{p}>0\right)$ and that no velocity diffusion is included in the face pressure expression $\left(K_{v}=0\right.$ for $\mathrm{AUSM}^{+}$-up). For this test case, the standard $\mathrm{AUSM}^{+}$-up scheme ( $K_{p}=0.25$ and $K_{v}=0.75$ ), the SLAU scheme and the momentum interpolation scheme fail.

For test 3 (see Figs. 37-46), all schemes studied produce a solution. For the AUSMtype schemes, it is again necessary to add pressure dissipation to the mass flux $\left(K_{p}>0\right)$ and to remove velocity diffusion in the face pressure definition $\left(K_{v}=0\right.$ for $\mathrm{AUSM}^{+}$-up ; compare Figs. 40-41 and Figs. 42-43). The presence of the inertia term in the mass flux definition of the AUSM-IT scheme is neutral for the quality of the solution. The quality of the AUSM-IT scheme is comparable to that of the $\mathrm{AUSM}^{+}$-up scheme with $K_{v}=0$. 


\begin{tabular}{|c|c|c|c|c|c|c|}
\hline Test & $\varrho_{\mathrm{L}}\left(\mathrm{kg} / \mathrm{m}^{3}\right)$ & $v_{\mathrm{L}}(\mathrm{m} / \mathrm{s})$ & $p_{\mathrm{L}}(\mathrm{Pa})$ & $\varrho_{\mathrm{R}}\left(\mathrm{kg} / \mathrm{m}^{3}\right)$ & $v_{\mathrm{R}}(\mathrm{m} / \mathrm{s})$ & $p_{\mathrm{R}}(\mathrm{Pa})$ \\
\hline 1 & 1 & 0.75 & 1 & 0.125 & 0 & 0.1 \\
2 & 1 & -19.59745 & 1000.0 & 1 & -19.59745 & 0.01 \\
3 & 0.445 & 0.698 & 3.528 & 0.5 & 0 & 0.571 \\
\hline
\end{tabular}

Table 2

Settings for the Riemann problems of Sec. 5.2.
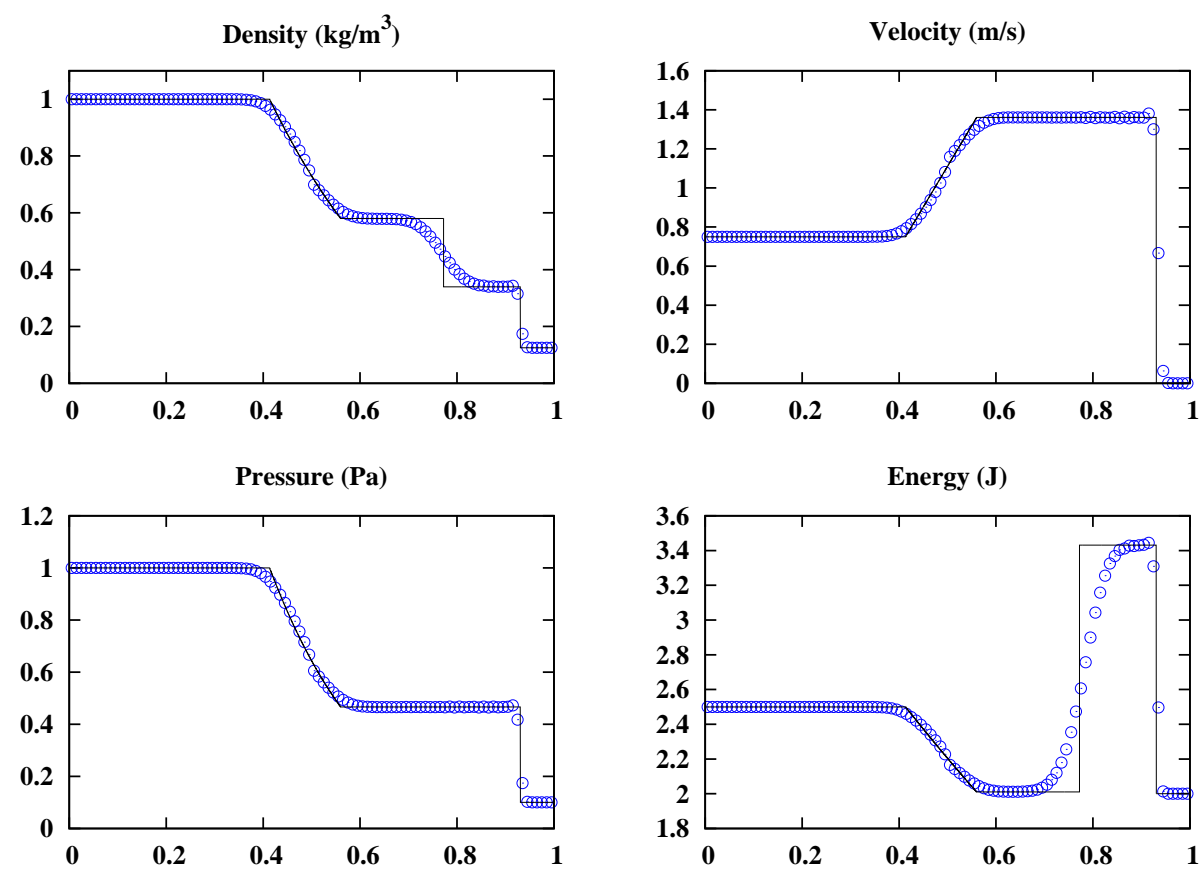

Fig. 22. Test 1 of Sec. 5.2. AUSM-IT scheme with $K_{p}=K_{I}=0.25$ (०) and exact (solid line) solutions at time $t=0.2 \mathrm{~s}$. 

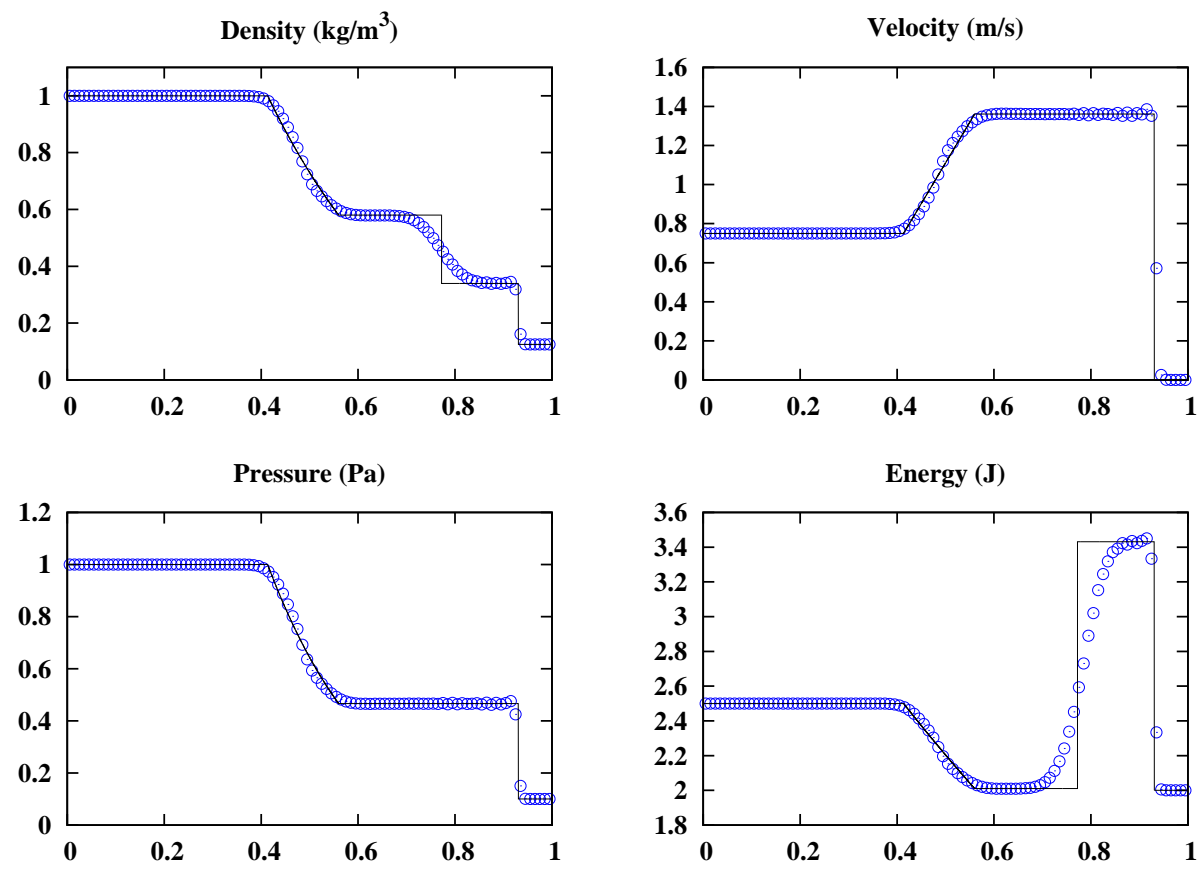

Fig. 23. Test 1 of Sec. 5.2. AUSM-IT scheme with $K_{p}=0$ and $K_{I}=0.25$ (०) and exact (solid line) solutions at time $t=0.2 \mathrm{~s}$.
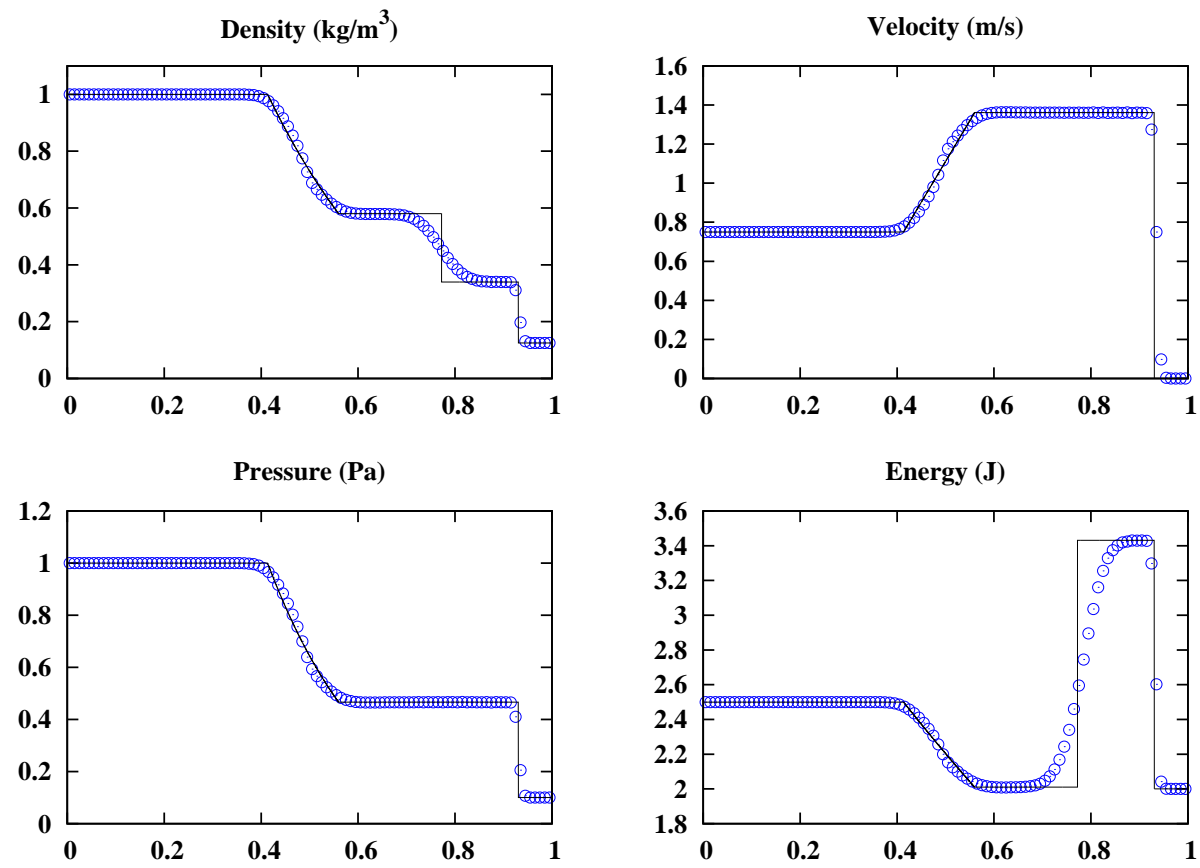

Fig. 24. Test 1 of Sec. 5.2. AUSM ${ }^{+}$scheme (o) and exact (solid line) solutions at time $t=0.2 \mathrm{~s}$. 

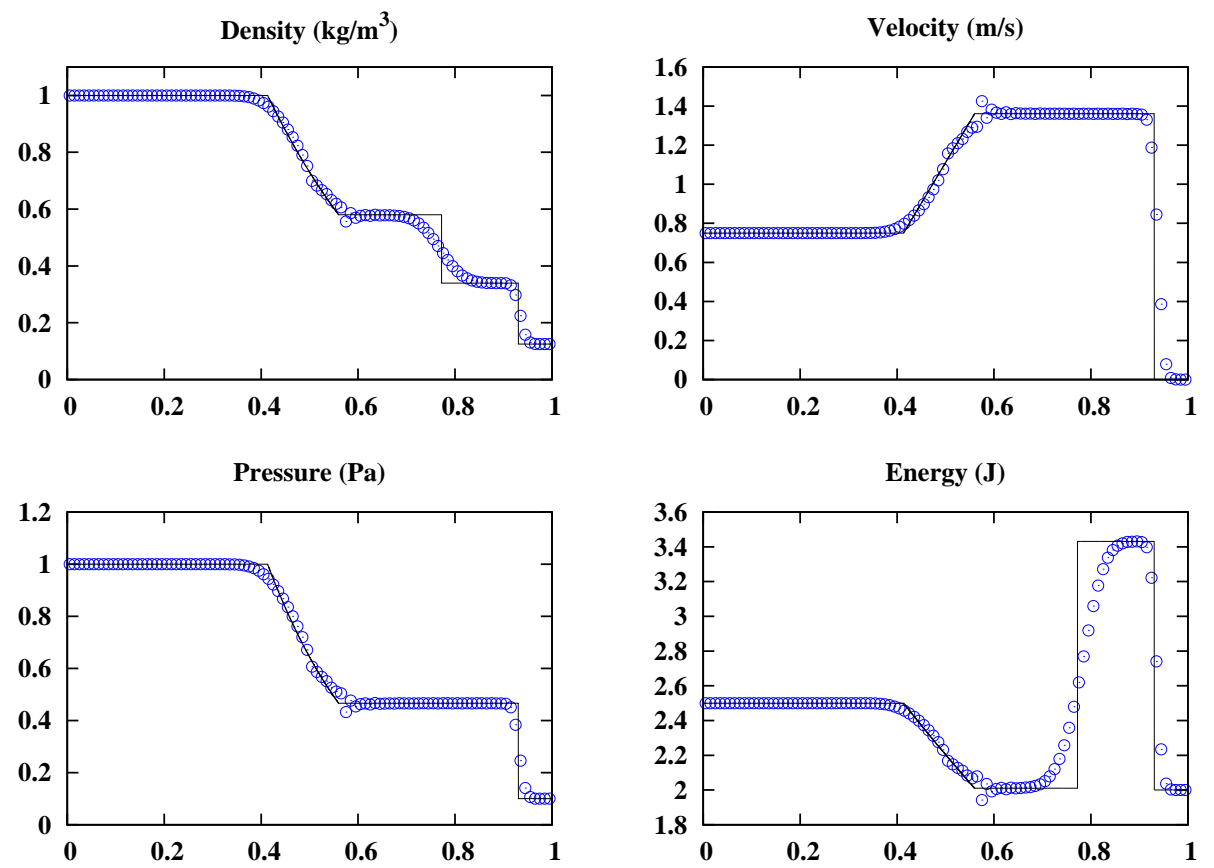

Fig. 25. Test 1 of Sec. 5.2. $\mathrm{AUSM}^{+}$-up scheme with $K_{p}=0.25, K_{v}=0.75$ and $f_{c}$ given by Eq. (24) (see Appendix A) (o), and exact (solid line) solutions at time $t=0.2 \mathrm{~s}$.
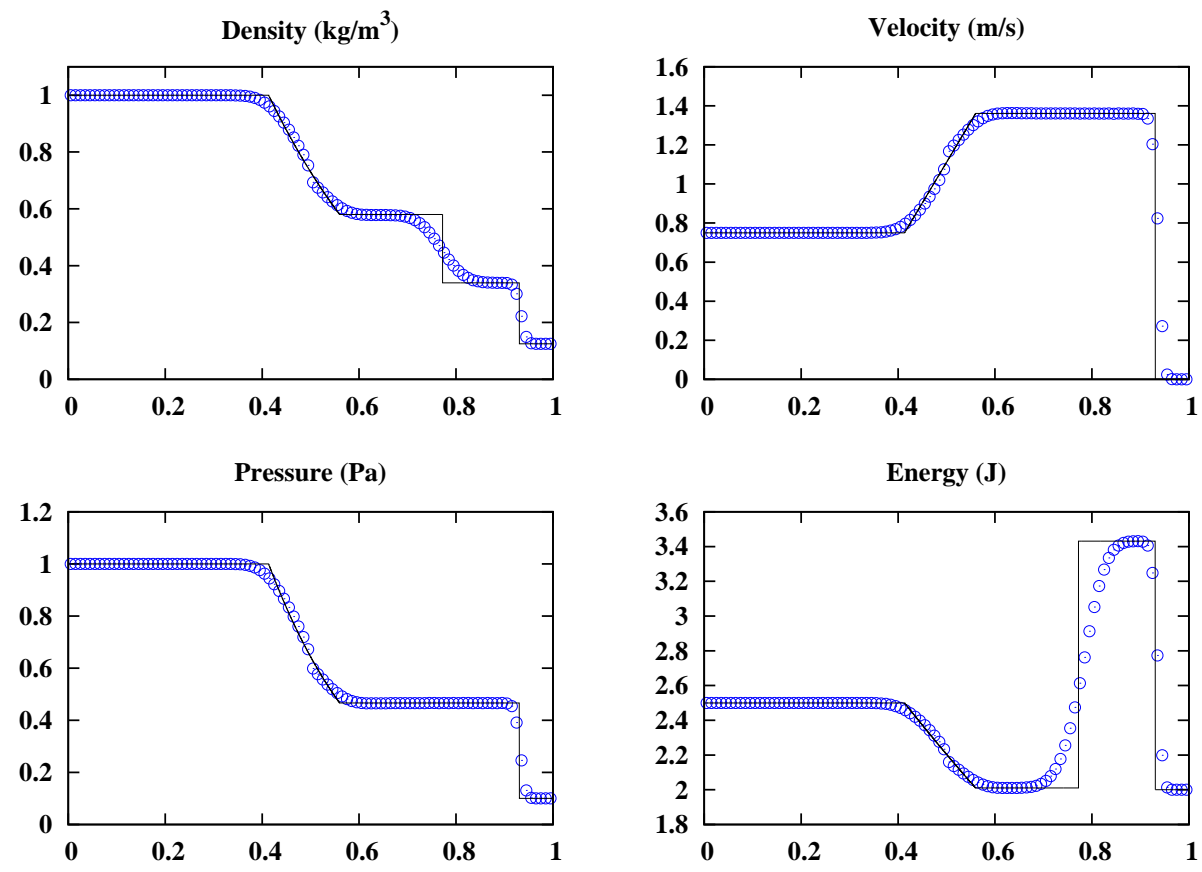

Fig. 26. Test 1 of Sec. 5.2. $\mathrm{AUSM}^{+}$-up scheme with $K_{p}=0.25, K_{v}=0$ and $f_{c}$ given by Eq. (24) (see Appendix A) (o), and exact (solid line) solutions at time $t=0.2 \mathrm{~s}$. 

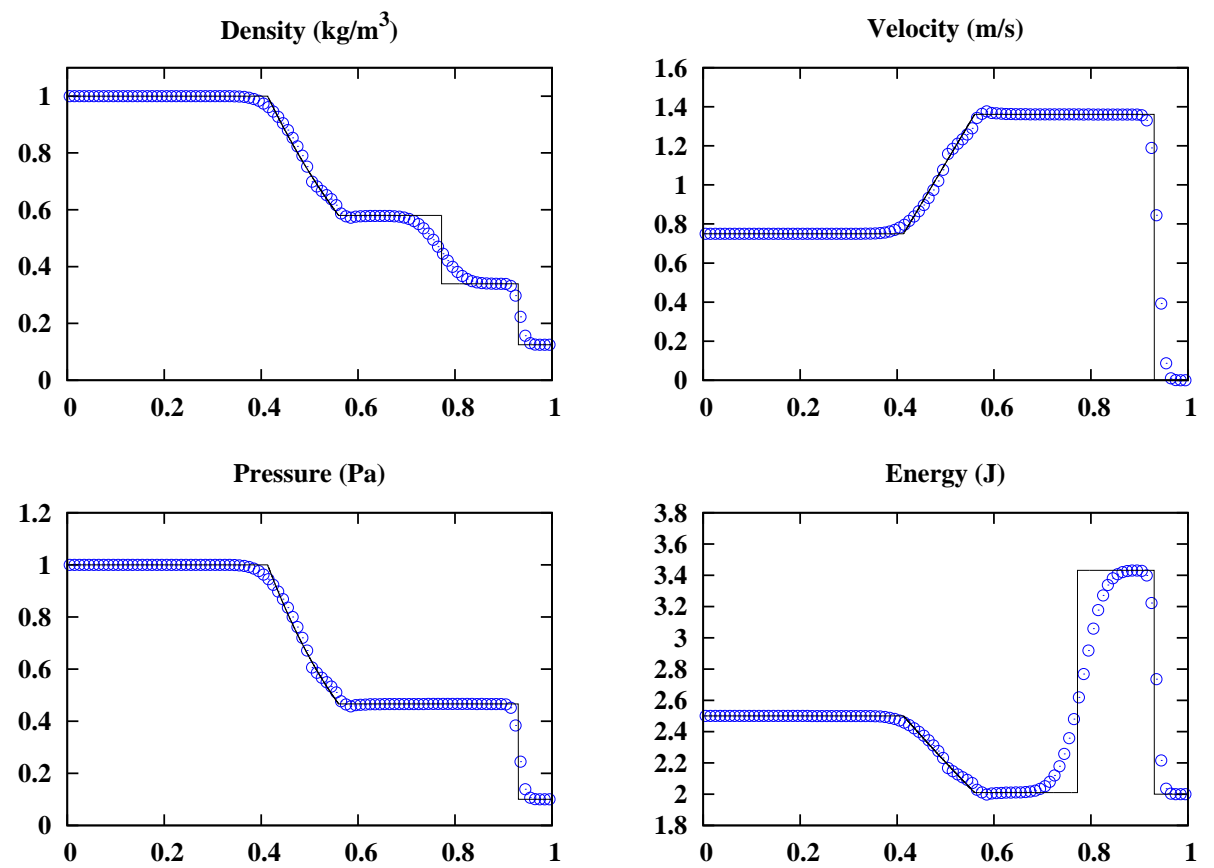

Fig. 27. Test 1 of Sec. 5.2. AUSM $^{+}$-up scheme with $K_{p}=0.25, K_{v}=0.75$ and $f_{c}=1$ (o), and exact (solid line) solutions at time $t=0.2 \mathrm{~s}$.
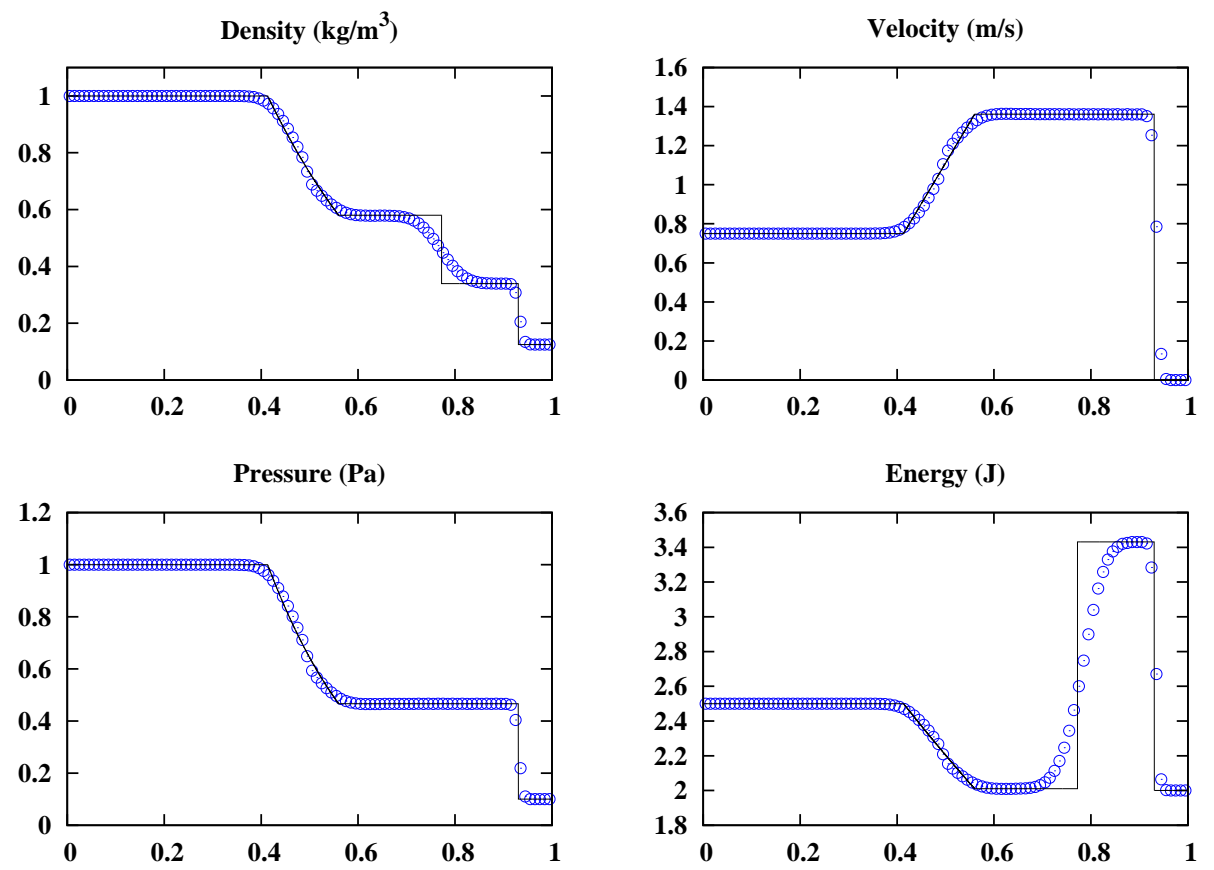

Fig. 28. Test 1 of Sec. 5.2. 'JCAM 2013' scheme (o) with $\beta=1$ (see Eq. (26)) and exact (solid line) solutions at time $t=0.2 \mathrm{~s}$. 

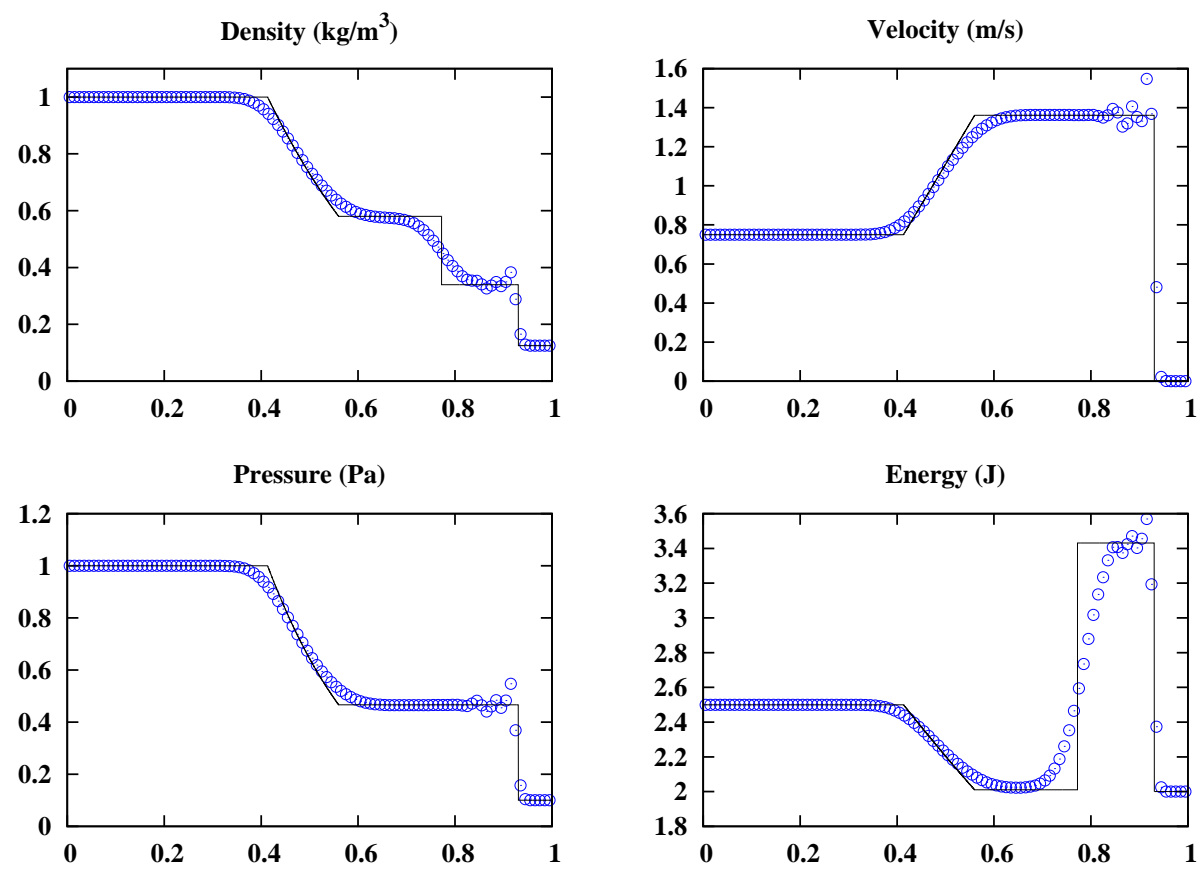

Fig. 29. Test 1 of Sec. 5.2. SLAU scheme (o) and exact (solid line) solutions at time $t=0.2 \mathrm{~s}$.
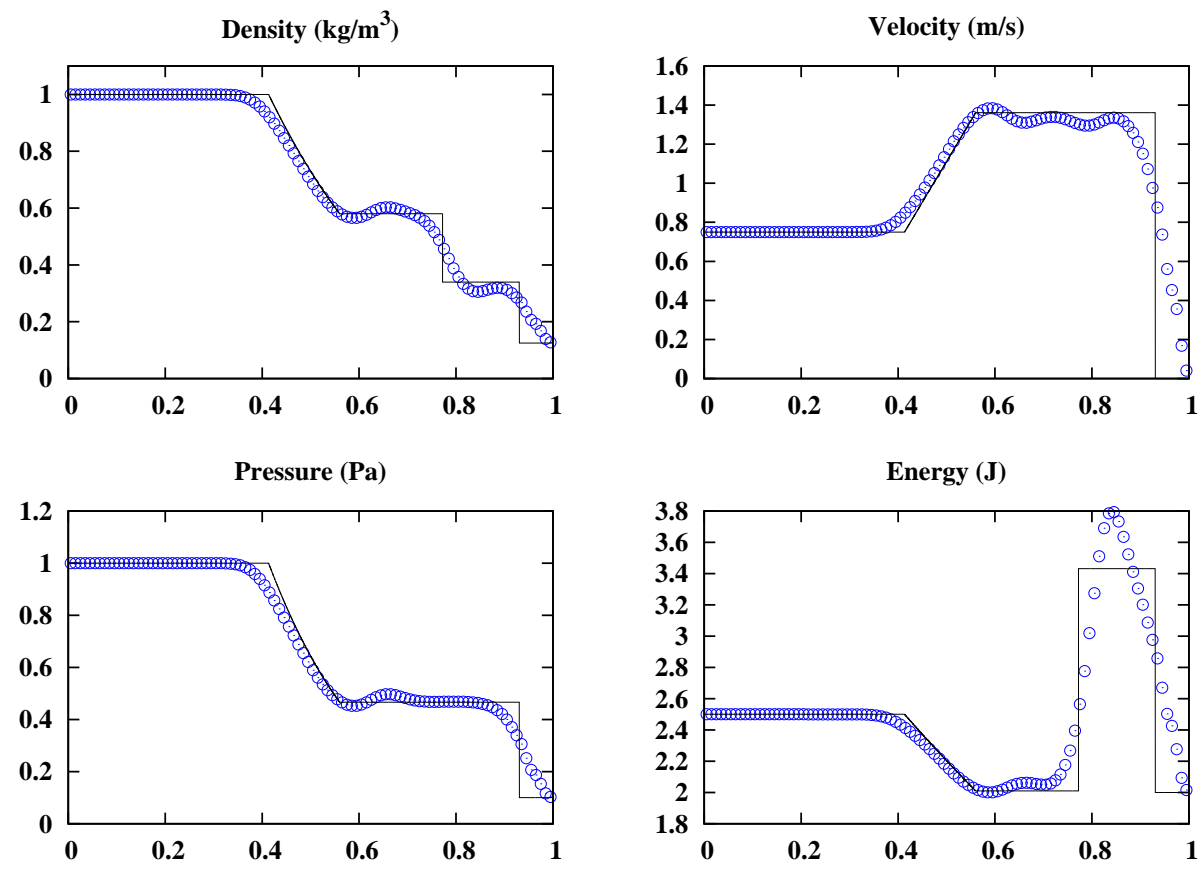

Fig. 30. Test 1 of Sec. 5.2. Momentum interpolation scheme (o) (see Eq. (29)) and exact (solid line) solutions at time $t=0.2 \mathrm{~s}$. 

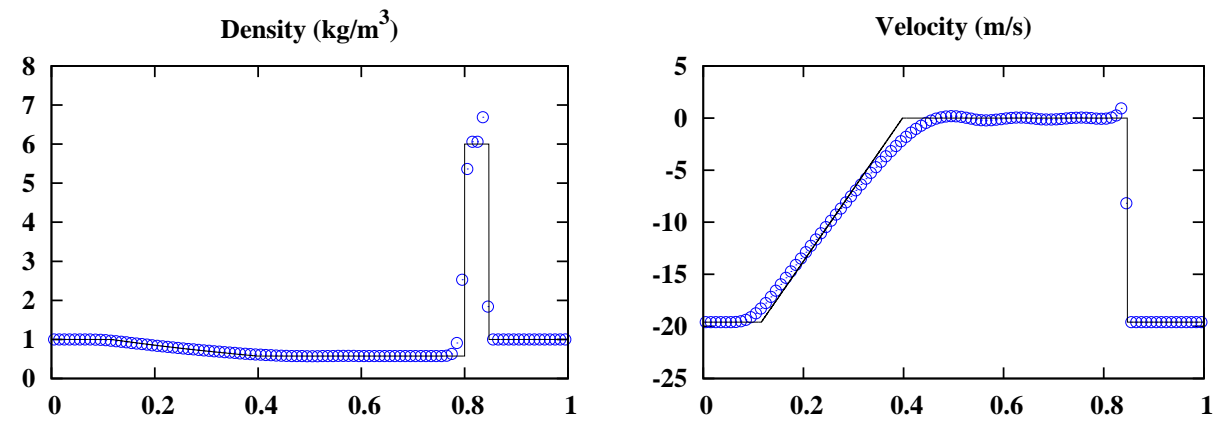

Pressure (Pa)
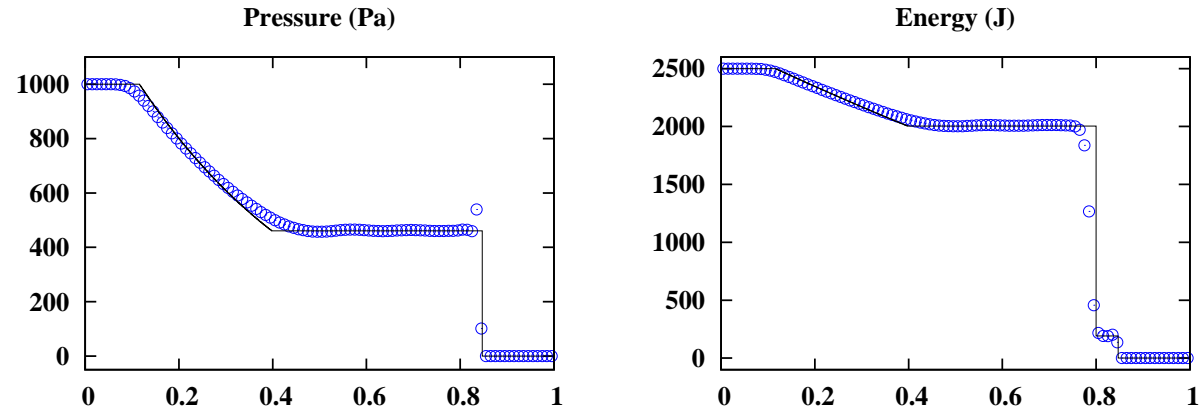

Fig. 31. Test 2 of Sec. 5.2. AUSM-IT scheme with $K_{p}=K_{I}=0.25$ (o) and exact (solid line) solutions at time $t=0.012 \mathrm{~s}$.
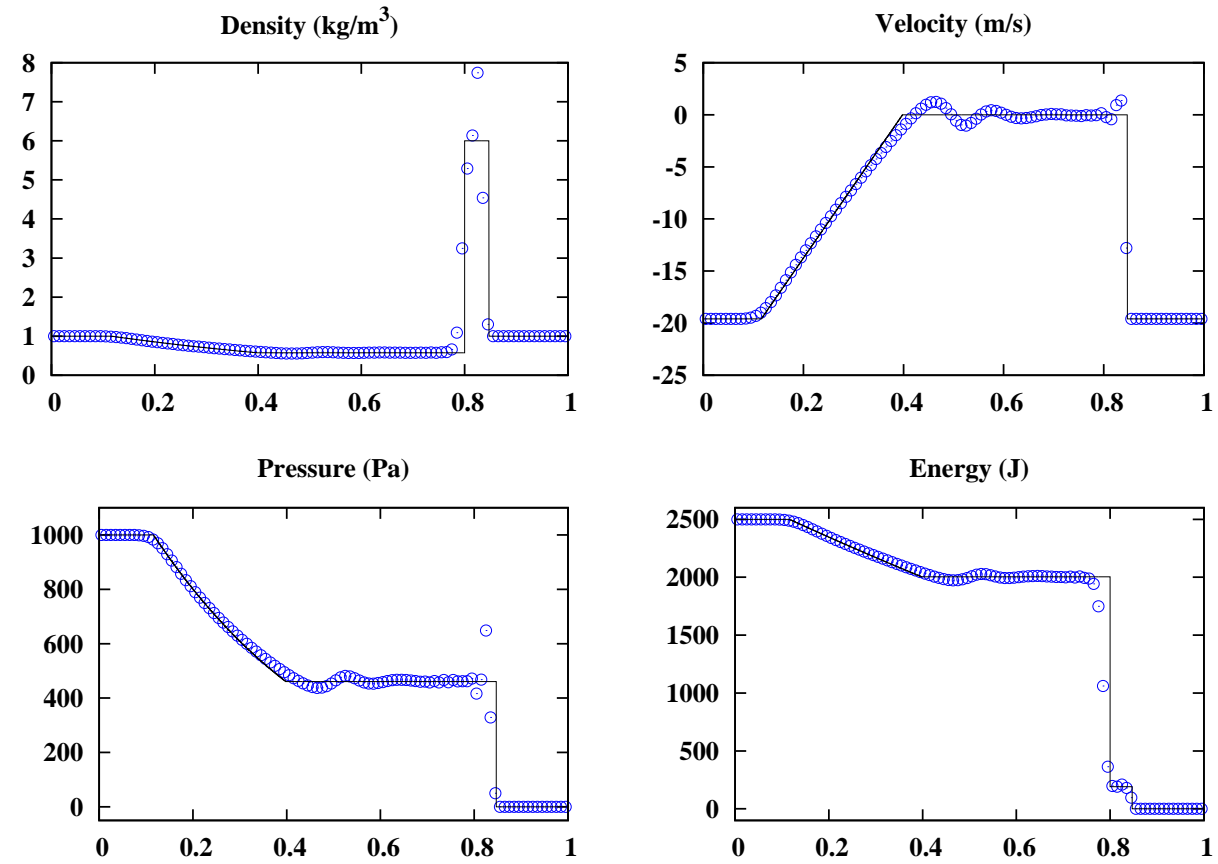

Fig. 32. Test 2 of Sec. 5.2. AUSM-IT scheme with $K_{p}=0$ and $K_{I}=0.25$ (०) and exact (solid line) solutions at time $t=0.012 \mathrm{~s}$. 

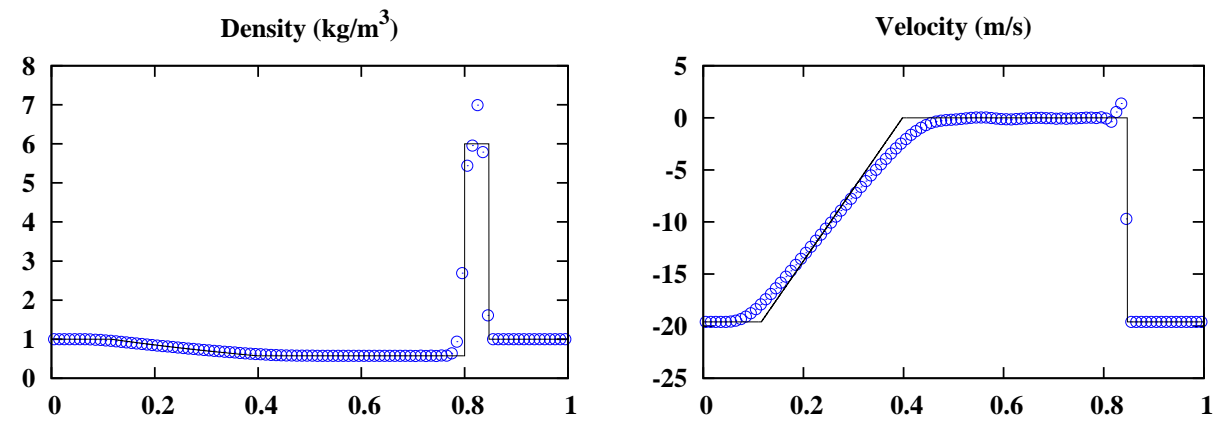

Pressure (Pa)
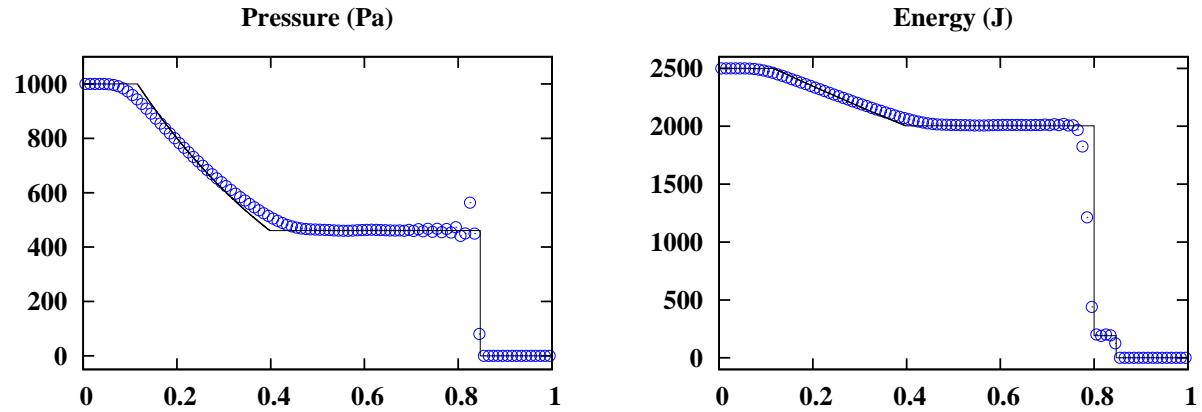

Fig. 33. Test 2 of Sec. 5.2. AUSM ${ }^{+}$scheme (o) and exact (solid line) solutions at time $t=0.012 \mathrm{~s}$.
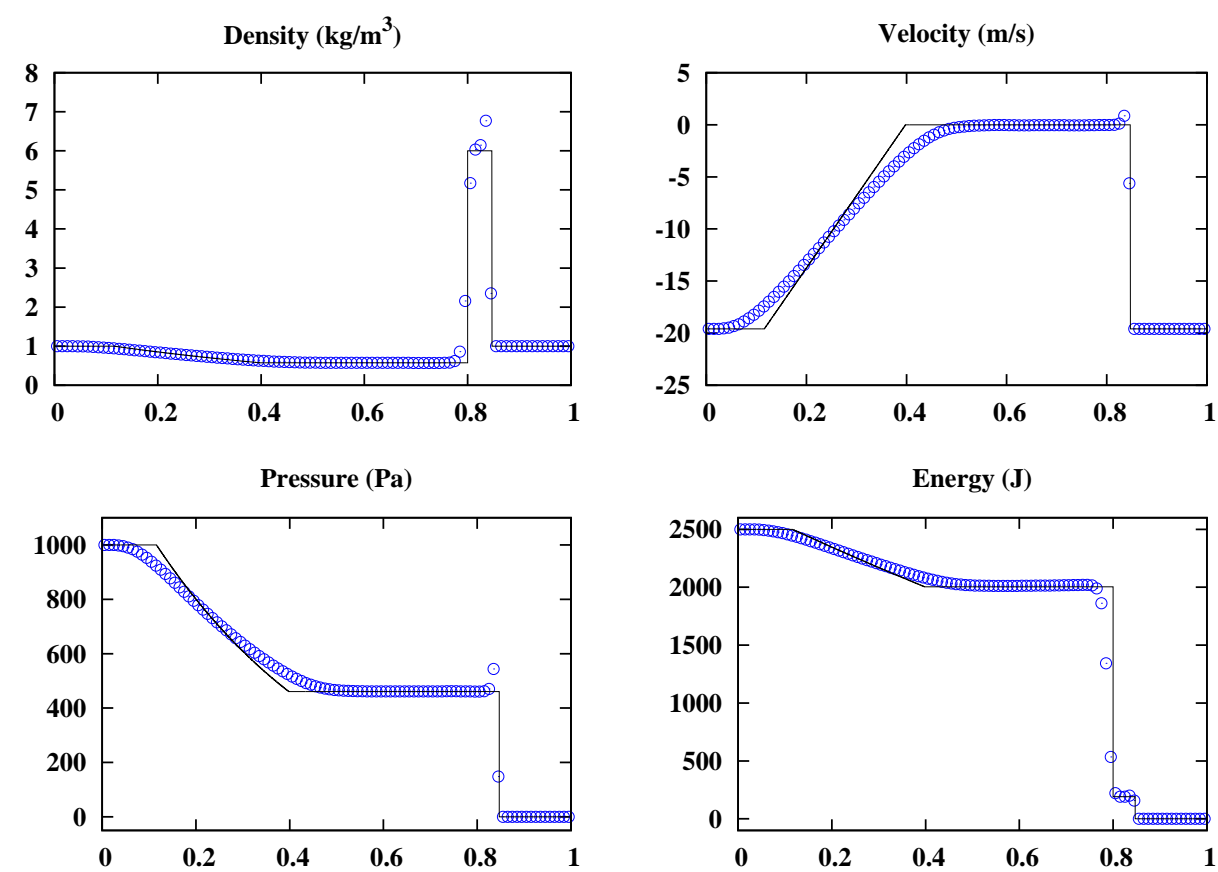

Fig. 34. Test 2 of Sec. 5.2. AUSM ${ }^{+}$-up scheme with $K_{p}=0.25, K_{v}=0$ and $f_{c}$ given by Eq. (24) (see Appendix A) (o), and exact (solid line) solutions at time $t=0.012 \mathrm{~s}$. 

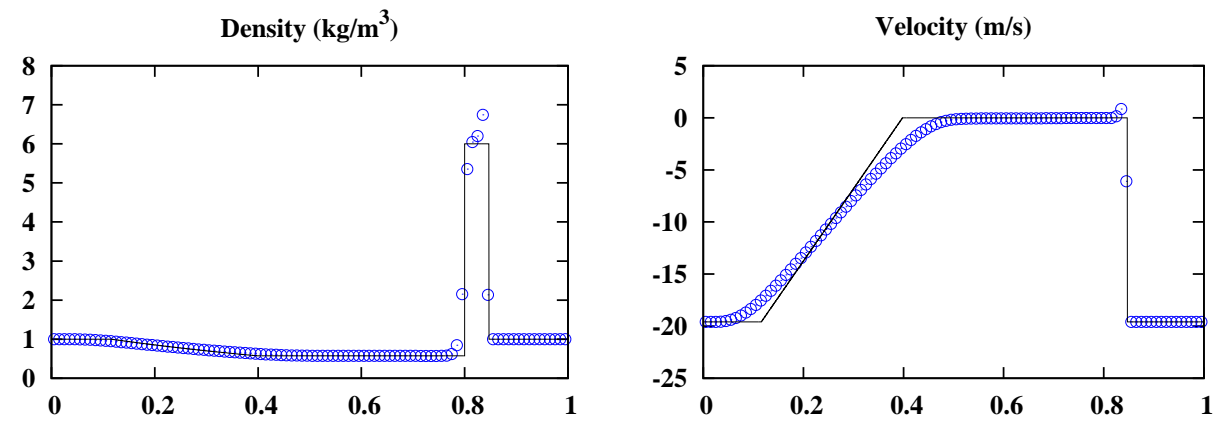

Pressure (Pa)
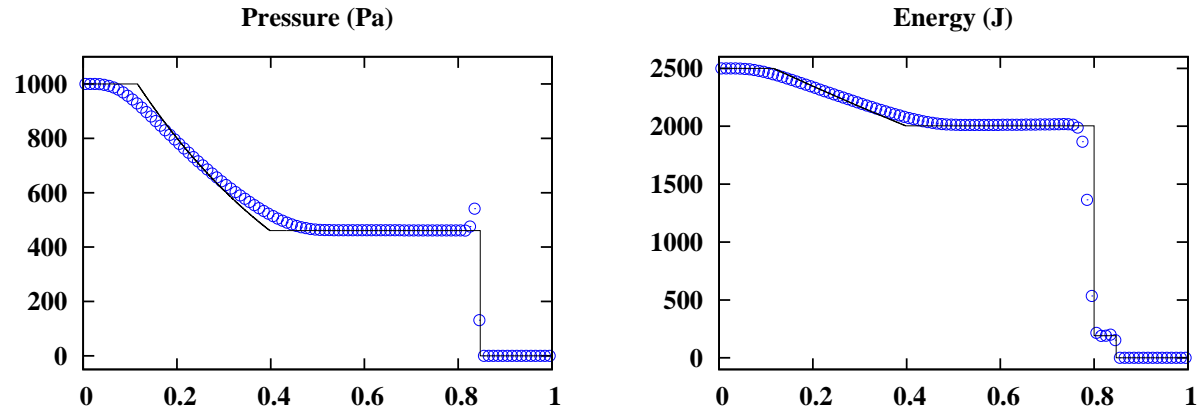

Fig. 35. Test 2 of Sec. 5.2. AUSM ${ }^{+}$-up scheme with $K_{p}=0.25, K_{v}=0$ and $f_{c}=1$ (०), and exact (solid line) solutions at time $t=0.012 \mathrm{~s}$.
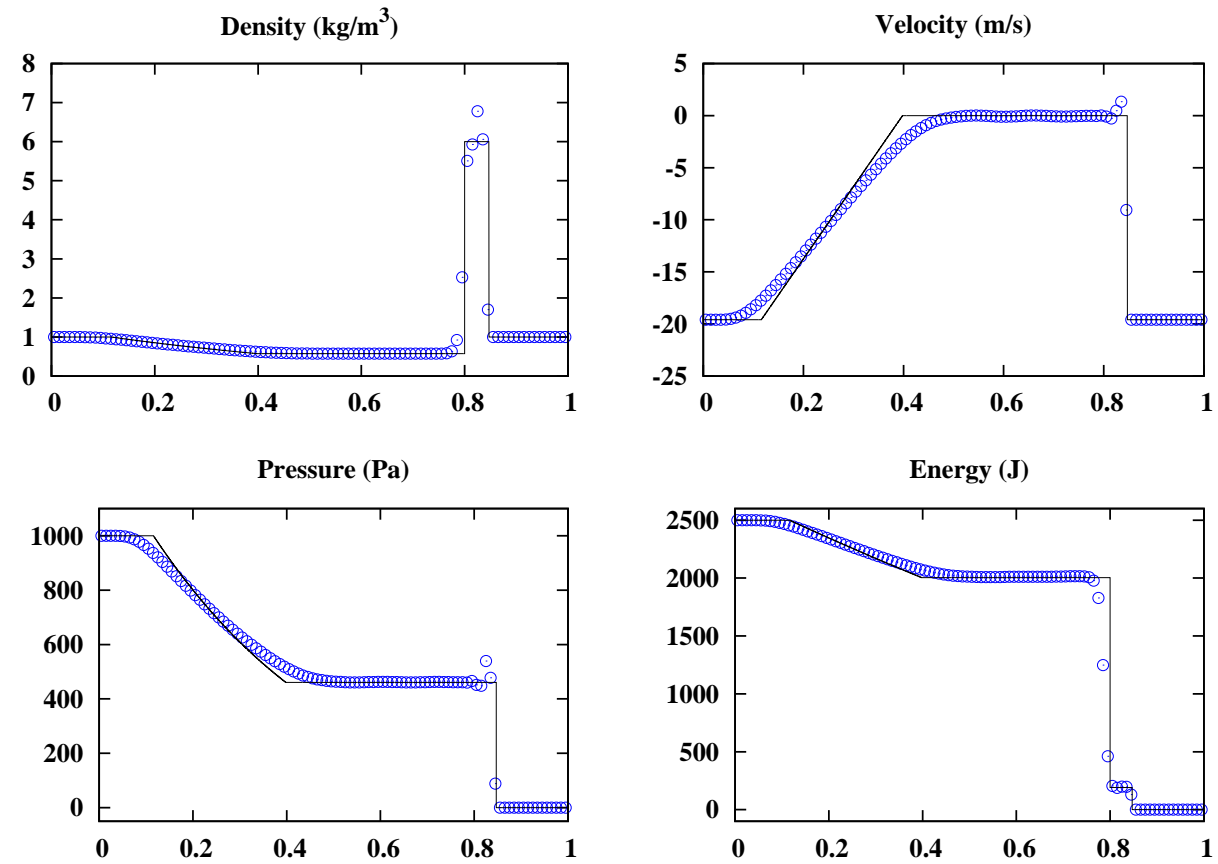

Fig. 36. Test 2 of Sec. 5.2. 'JCAM 2013' scheme (o) with $\beta=1$ (see Eq. (26)) and exact (solid line) solutions at time $t=0.012 \mathrm{~s}$. 

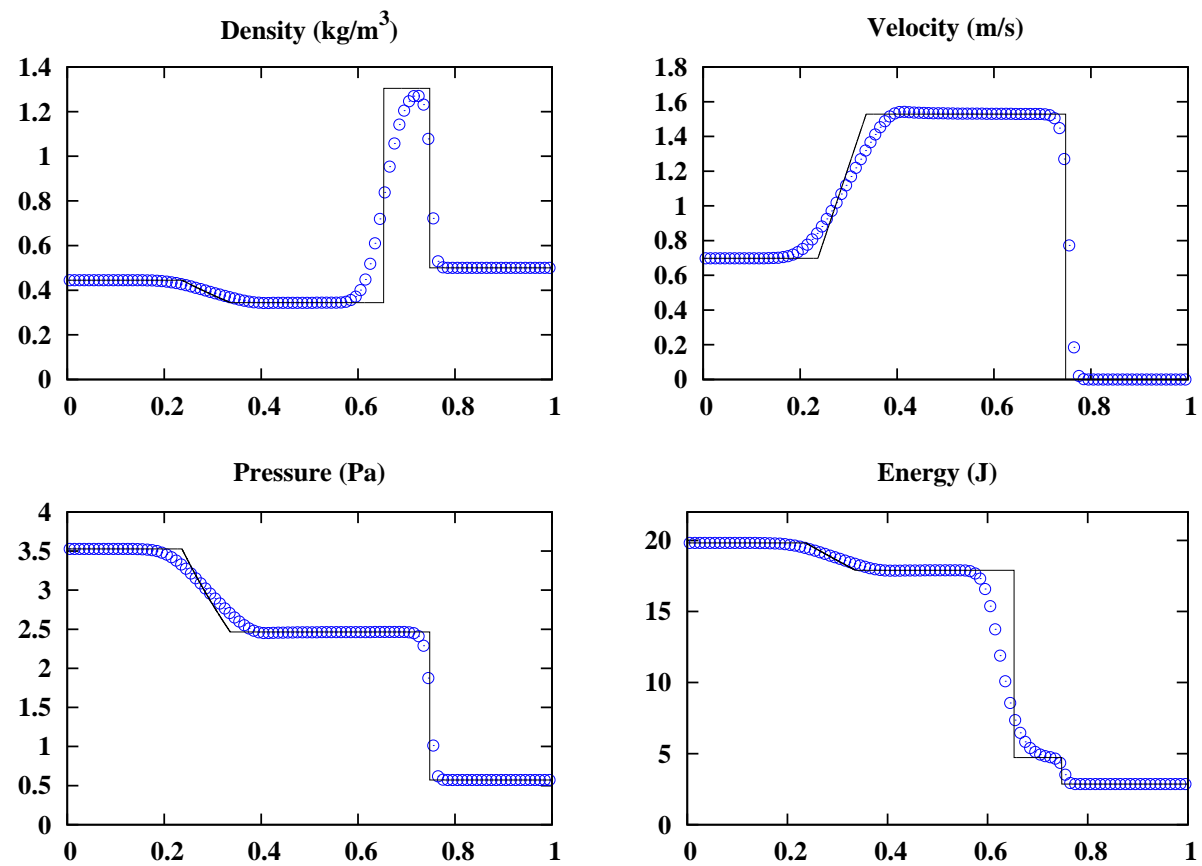

Fig. 37. Test 3 of Sec. 5.2. AUSM-IT scheme with $K_{p}=0.25$ and $K_{I}=0.25$ (०) and exact (solid line) solutions at time $t=0.1 \mathrm{~s}$.
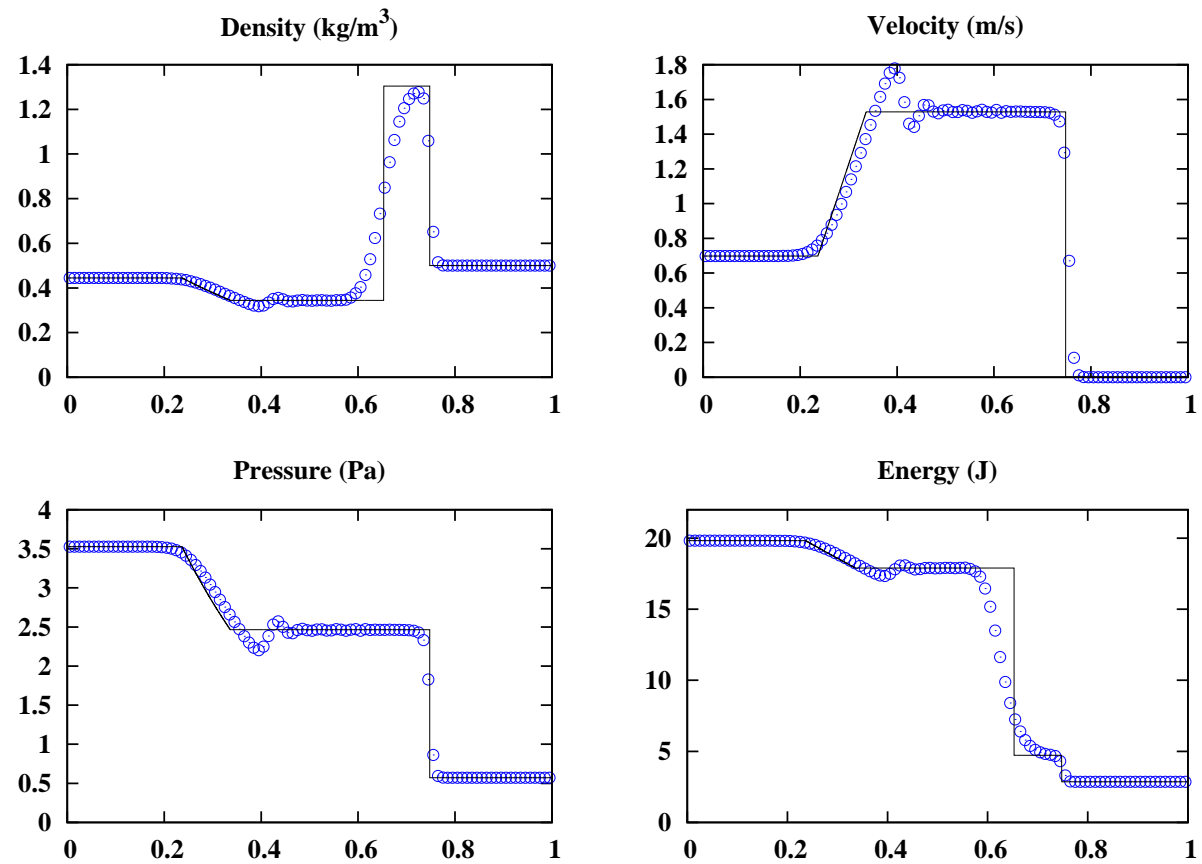

Fig. 38. Test 3 of Sec. 5.2. AUSM-IT scheme with $K_{p}=0$ and $K_{I}=0.25$ (०) and exact (solid line) solutions at time $t=0.1 \mathrm{~s}$. 

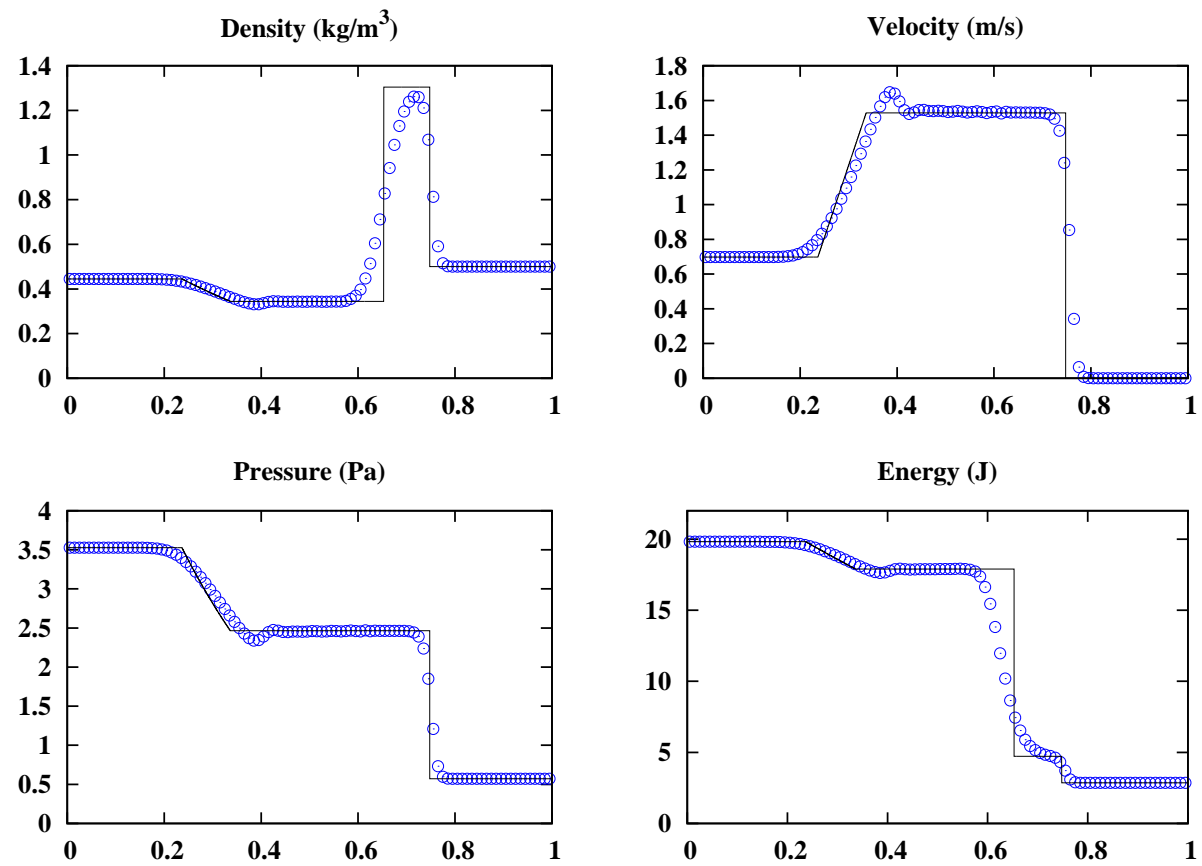

Fig. 39. Test 3 of Sec. 5.2. AUSM ${ }^{+}$scheme (o) and exact (solid line) solutions at time $t=0.1 \mathrm{~s}$.
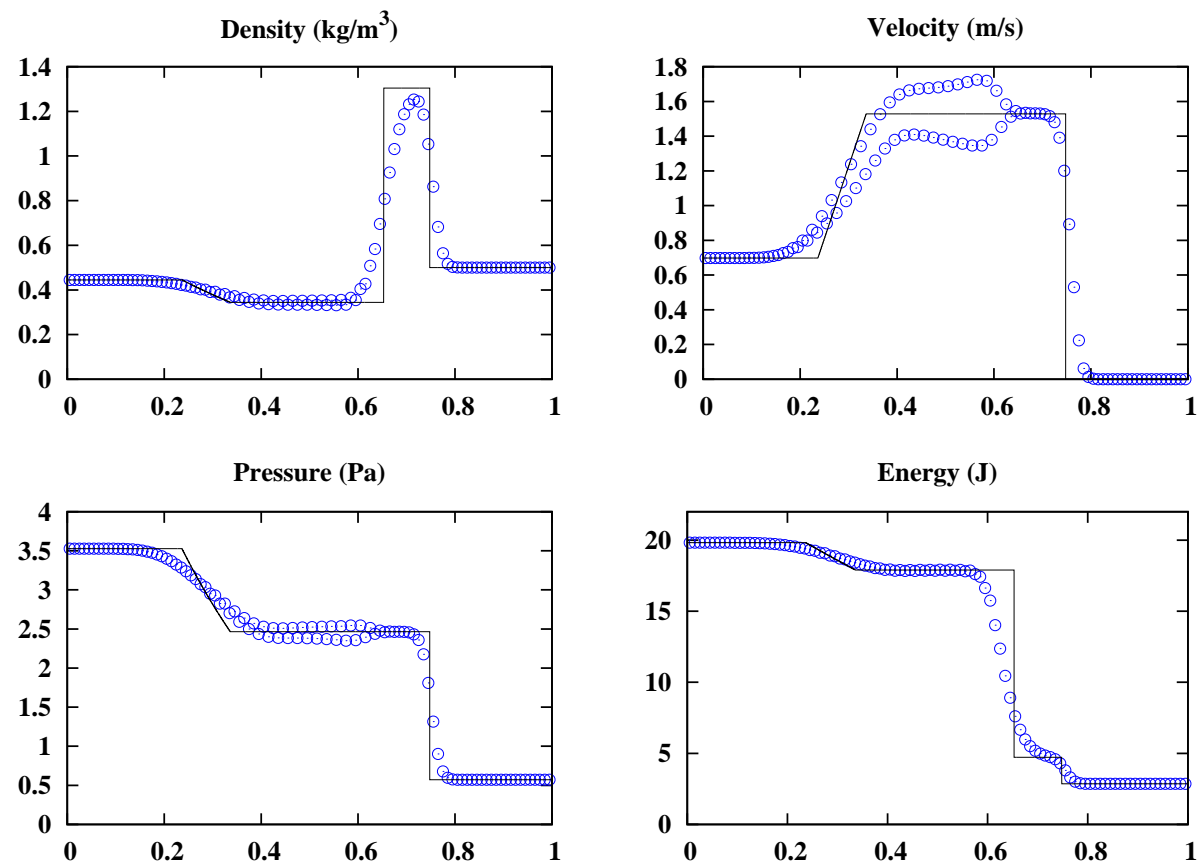

Fig. 40. Test 3 of Sec. 5.2. AUSM ${ }^{+}$-up scheme with $K_{p}=0.25, K_{v}=0.75$ and $f_{c}$ given by Eq. (24) (see Appendix A) (o), and exact (solid line) solutions at time $t=0.1 \mathrm{~s}$. 

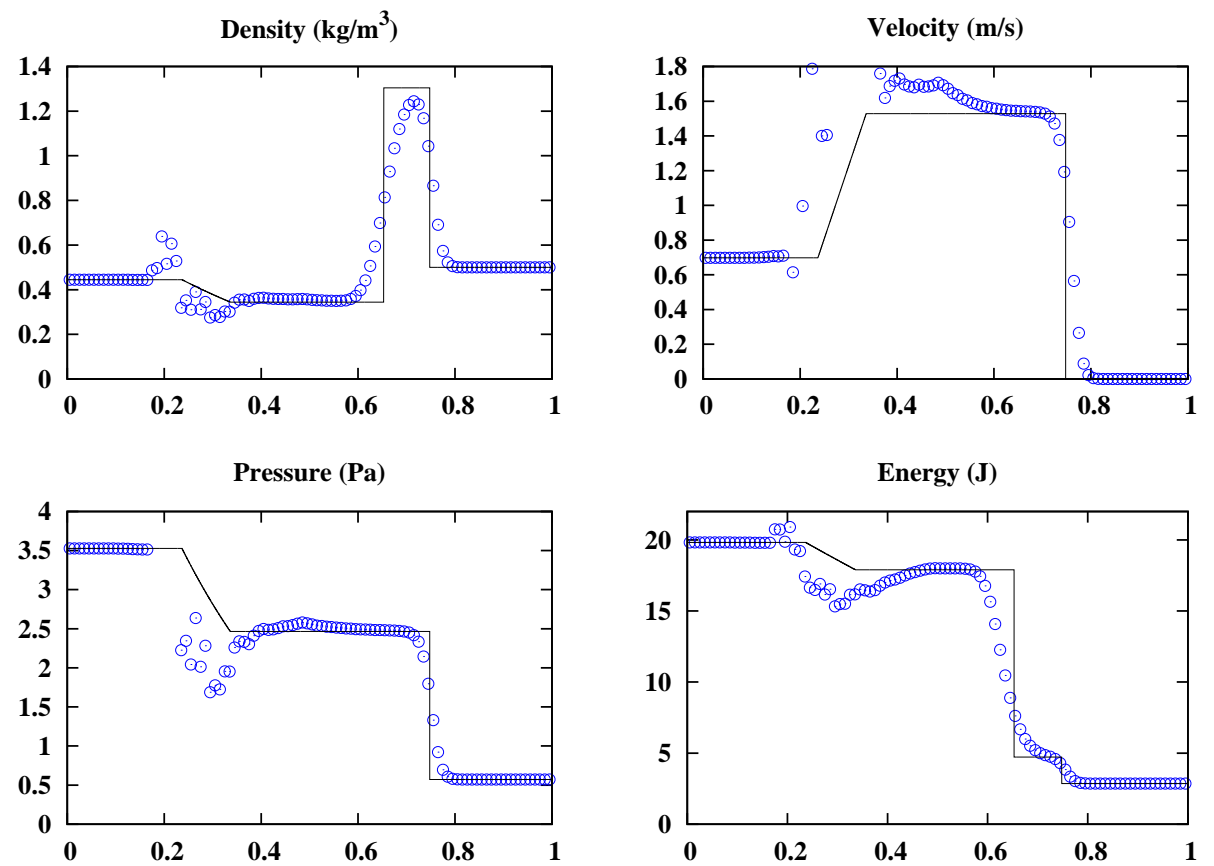

Fig. 41. Test 3 of Sec. 5.2. AUSM $^{+}$-up scheme with $K_{p}=0.25, K_{v}=0.75$ and $f_{c}=1$ (o), and exact (solid line) solutions at time $t=0.1 \mathrm{~s}$.
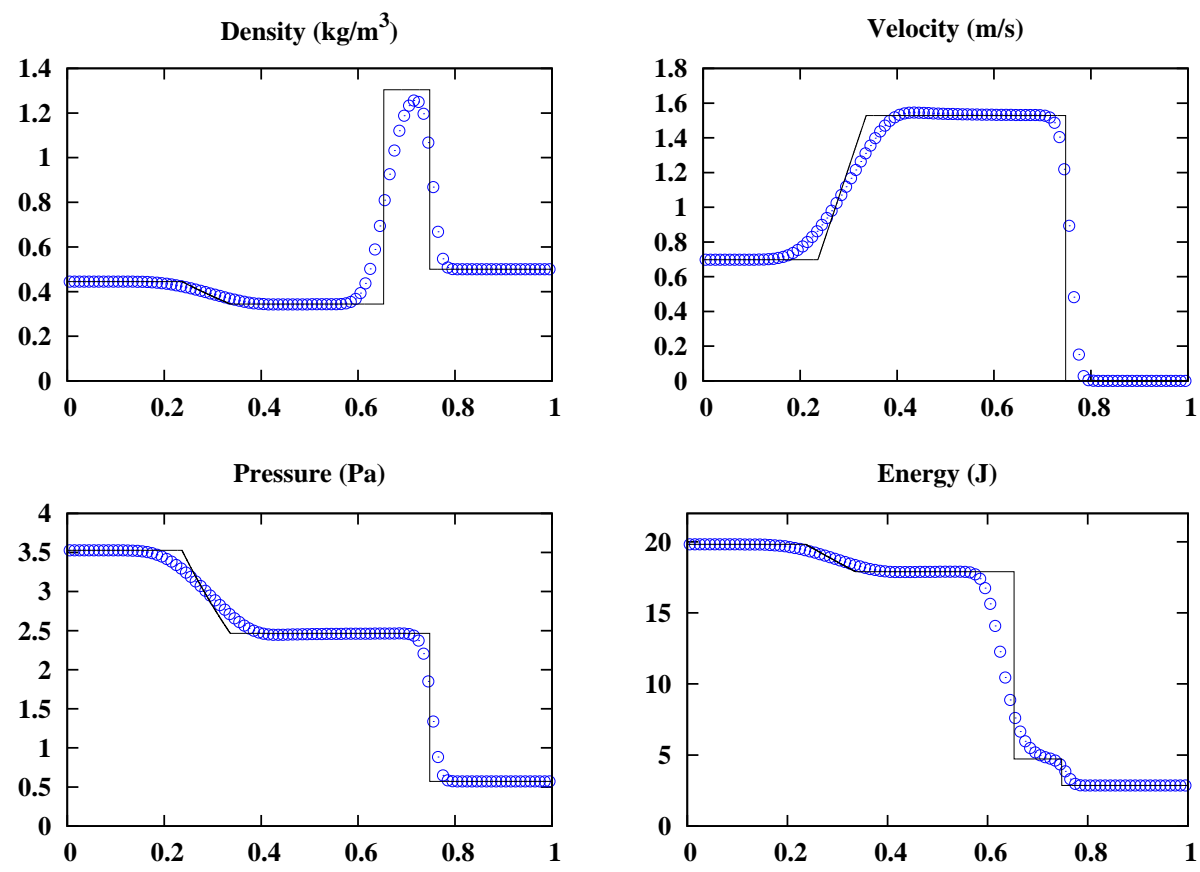

Fig. 42. Test 3 of Sec. 5.2. $\mathrm{AUSM}^{+}$-up scheme with $K_{p}=0.25, K_{v}=0$ and $f_{c}$ given by Eq. (24) (see Appendix A) (o), and exact (solid line) solutions at time $t=0.1 \mathrm{~s}$. 

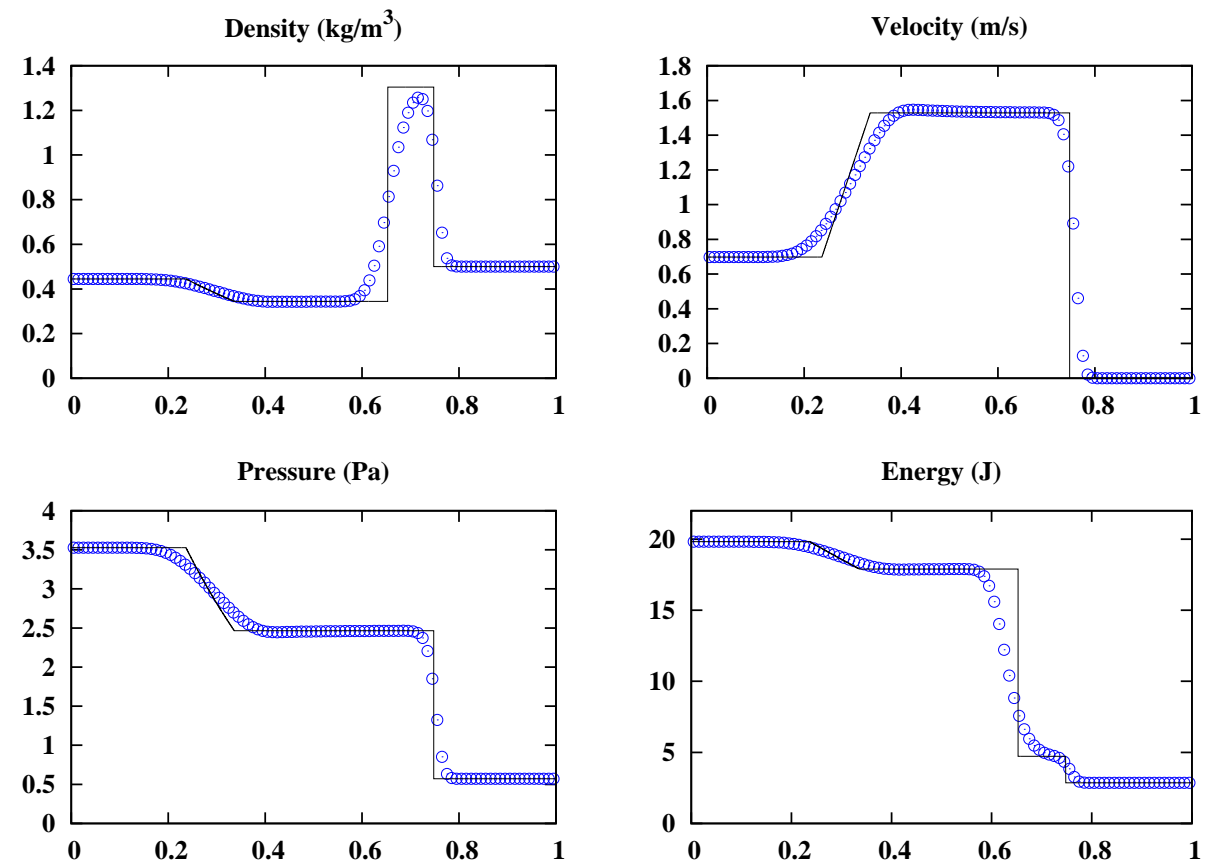

Fig. 43. Test 3 of Sec. 5.2. AUSM ${ }^{+}$-up scheme with $K_{p}=0.25, K_{v}=0$ and $f_{c}=1$ (०), and exact (solid line) solutions at time $t=0.1 \mathrm{~s}$.
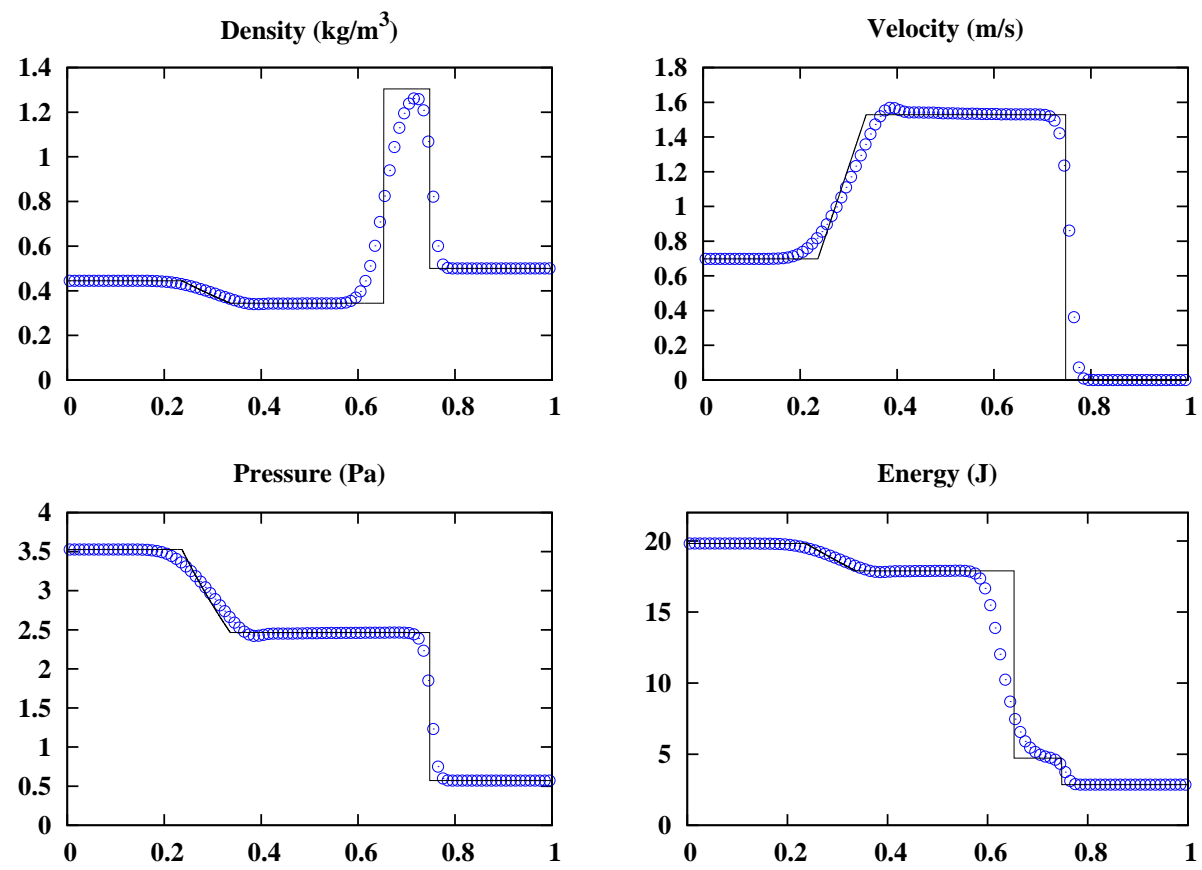

Fig. 44. Test 3 of Sec. 5.2. 'JCAM 2013' scheme (o) with $\beta=1$ (see Eq. (26)) and exact (solid line) solutions at time $t=0.1 \mathrm{~s}$. 

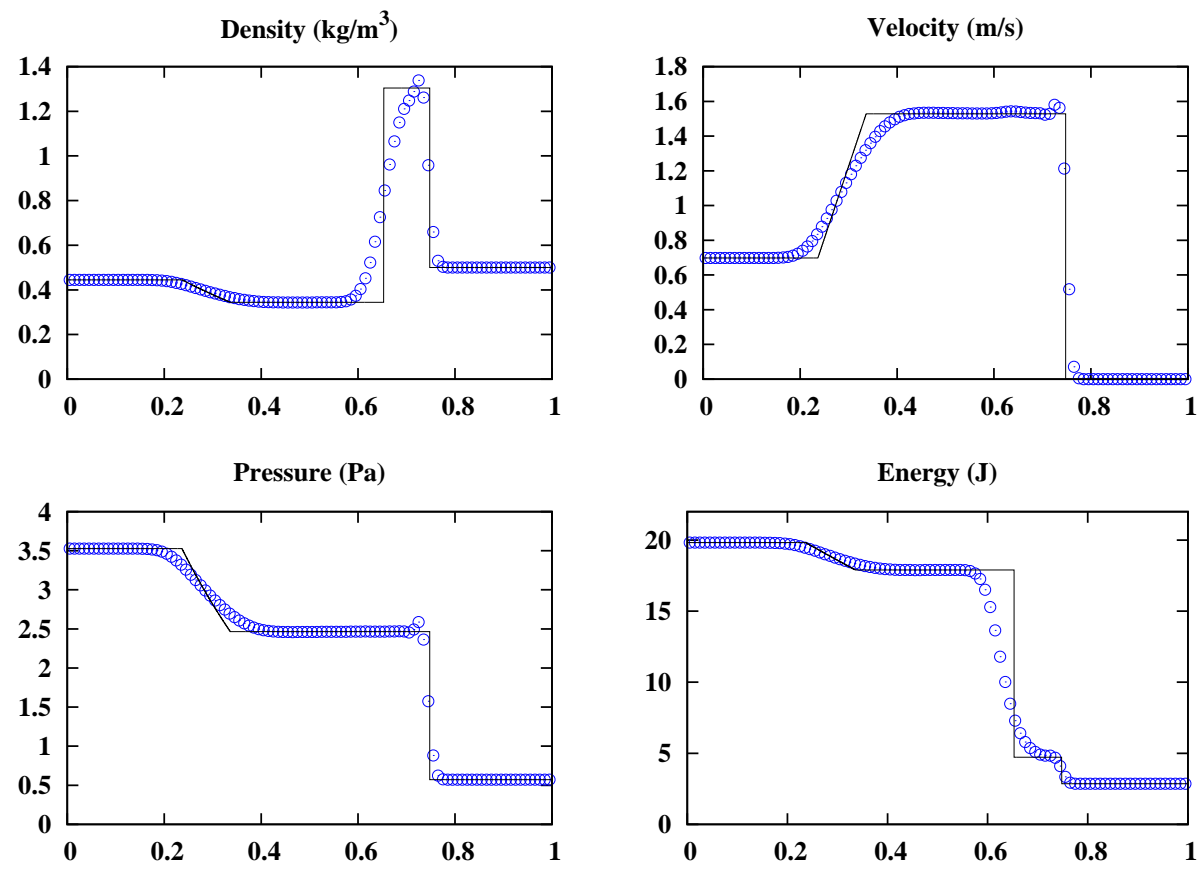

Fig. 45. Test 3 of Sec. 5.2. SLAU scheme (o) and exact (solid line) solutions at time $t=0.1 \mathrm{~s}$.
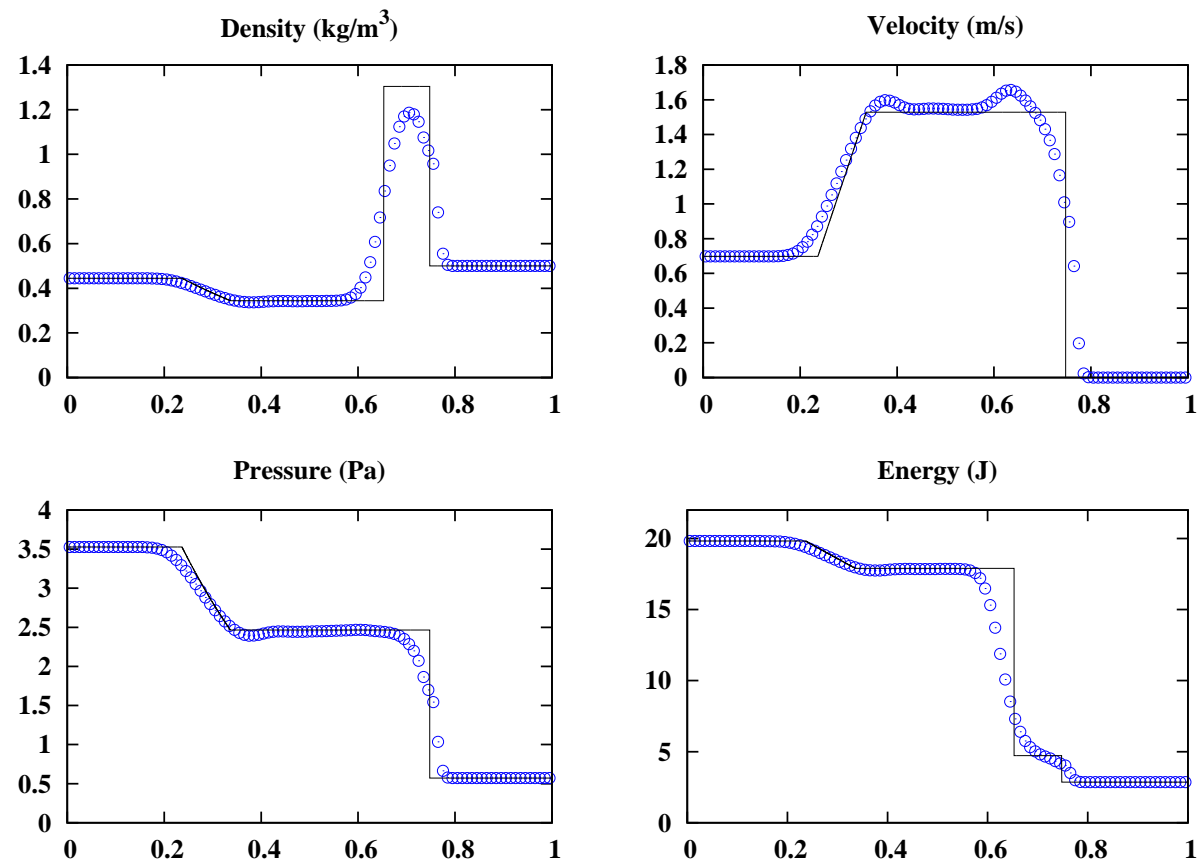

Fig. 46. Test 3 of Sec. 5.2. Momentum interpolation scheme (o) (see Eq. (29)) and exact (solid line) solutions at time $t=0.1 \mathrm{~s}$. 
From the previous numerical experiments the following typical values of the parameters are suggested:

$$
\sigma=1, K_{I}=K_{p}=0.25
$$

Moreover, no velocity diffusion is added in the face pressure $\left(K_{v}=0\right.$, referring to the $\mathrm{AUSM}^{+}$-up scheme, see Eq. (25)).

\section{Conclusion}

The proposed approach essentially consists in the introduction of an inertia term in the face velocity expression. It was applied in the present study to the AUSM+ ${ }^{+}$ up scheme by Liou [11]. The resulting scheme, called AUSM-IT (IT for Inertia Term), allows full Mach number range calculations, since it is an extension of the $\mathrm{AUSM}^{+}$-up scheme which is retrieved when the local Mach number is larger than unity.

The high quality of the results obtained for acoustic waves in low Mach number flows is about the same as by momentum interpolation. The reason for this good performance is the way the inertia term is introduced in the Godunov-type scheme. For periodic boundary conditions, it allows the conservation of the discrete linear acoustic energy in the low Mach number limit, at the first order. This is achieved if the way of interpolation of pressure tends to the central interpolation in the limit $M_{r} \rightarrow 0$. This acoustic energy conservation property at the discrete level conforms to the continuous asymptotic analysis. The superiority of the proposed Godunovtype scheme over the momentum interpolation technique for Riemann problems was numerically demonstrated.

Taking into account an inertia term for velocity interpolation is applicable in principle to any Godunov-type scheme. Thus, the approach proposed in the present study to design the AUSM-IT scheme from the $\mathrm{AUSM}^{+}$-up scheme can be applied to any other Godunov-type scheme, in order to obtain full Mach number range high quality results.

\section{Appendix A AUSM $^{+}$-up, AUSM ${ }^{+}$,'JCAM 2103', SLAU and momentum in- terpolation}

3-D version, except momentum interpolation, for sake of presentation. 
Notations:

$n$ : unit normal to the face

$$
\begin{aligned}
& V_{n}^{+}=V_{\mathrm{L}} \cdot n, V_{n}^{-}=V_{\mathrm{R}} \cdot n, M_{\mathrm{L}}=\frac{V_{n}^{+}}{c_{1 / 2}}, M_{\mathrm{R}}=\frac{V_{n}^{-}}{c_{1 / 2}} \\
& f_{p, \alpha}^{ \pm}(m)= \begin{cases}\frac{1}{2}(1 \pm \operatorname{sign}(m)) & ,|m| \geq 1 \\
\frac{1}{4}(m \pm 1)^{2}(2 \mp m) \pm \alpha m\left(m^{2}-1\right)^{2} & ,|m|<1\end{cases}
\end{aligned}
$$

\section{A.1 AUSM $^{+}$[10], AUSM ${ }^{+}$-up [11] and 'JCAM 2013' [14]}

The $\mathrm{AUSM}^{+}$-up scheme is first considered.

- Transporting face velocity:

$$
\begin{aligned}
& v_{1 / 2}^{\mathrm{T}}=c_{1 / 2} M_{1 / 2}-\frac{K_{p}}{\bar{\varrho} c_{1 / 2} f_{c}\left(M_{0}\right)} \max \left\{1-\sigma \bar{M}^{2}, 0\right\}\left(p_{\mathrm{R}}-p_{\mathrm{L}}\right) \\
& M_{1 / 2}=f_{M}^{+}\left(M_{\mathrm{L}}\right)+f_{M}^{-}\left(M_{\mathrm{R}}\right) \\
& f_{M}^{ \pm}(m)= \begin{cases}\frac{1}{2}(m \pm|m|), \\
\pm \frac{1}{4}(m \pm 1)^{2} \pm \frac{1}{8}\left(m^{2}-1\right)^{2}, \quad|m|<1\end{cases} \\
& \bar{\varrho}=\frac{\varrho_{\mathrm{L}}+\varrho_{\mathrm{R}}}{2} \\
& f_{c}\left(M_{0}\right)=M_{0}\left(2-M_{0}\right), M_{0}^{2}=\min \left\{1, \max \left\{\bar{M}^{2}, M_{\infty}^{2}\right\}\right\} \\
& \bar{M}^{2}=\frac{\left(V_{n}^{+}\right)^{2}+\left(V_{n}^{-}\right)^{2}}{2 c_{1 / 2}^{2}} \\
& c_{1 / 2}=\min \left\{\tilde{c}_{\mathrm{L}}, \tilde{c}_{\mathrm{R}}\right\}, \tilde{c}_{\mathrm{L}}=\left(c^{\star}\right)^{2} / \max \left\{c^{\star}, V_{n}^{+}\right\}, \tilde{c}_{\mathrm{R}}=\left(c^{\star}\right)^{2} / \max \left\{c^{\star},-V_{n}^{-}\right\} \\
& \left(c^{\star}\right)^{2}=\frac{2(\gamma-1)}{\gamma+1} H \\
& 0 \leq K_{p} \leq 1, \sigma \leq 1 ; \text { typically: } K_{p}=0.25, \sigma=1
\end{aligned}
$$

- Face pressure:

$$
\begin{aligned}
p_{1 / 2}=f_{p, \alpha}^{+}( & \left.M_{\mathrm{L}}\right) p_{\mathrm{L}}+f_{p, \alpha}^{-}\left(M_{\mathrm{R}}\right) p_{\mathrm{R}} \\
& \quad-K_{v} f_{p, \alpha}^{+}\left(M_{\mathrm{L}}\right) f_{p, \alpha}^{-}\left(M_{\mathrm{R}}\right)\left(\varrho_{\mathrm{L}}+\varrho_{\mathrm{R}}\right) f_{c}\left(M_{0}\right) c_{1 / 2}\left(V_{n}^{-}-V_{n}^{+}\right)
\end{aligned}
$$

where

$$
f_{p, \alpha}^{ \pm}(m)= \begin{cases}\frac{1}{2}(1 \pm \operatorname{sign}(m)) & ,|m| \geq 1 \\ \frac{1}{4}(m \pm 1)^{2}(2 \mp m) \pm \alpha m\left(m^{2}-1\right)^{2} & ,|m|<1\end{cases}
$$




$$
\alpha=\frac{3}{16}\left[-4+5\left(f_{c}\left(M_{0}\right)\right)^{2}\right] \quad, \quad 0 \leq K_{v} \leq 1 ; \text { typically: } K_{v}=0.75
$$

- Convected quantities: upwinded

- The $\mathrm{AUSM}^{+}$scheme is obtained from the $\mathrm{AUSM}^{+}$-up scheme by taking $K_{p}=0$ in Eq. (23), and $K_{v}=0$ along with $\alpha=3 / 16$ in Eq. (25). Note that the interface speed of sound retained for $\mathrm{AUSM}^{+}$is the same as the one for $\mathrm{AUSM}^{+}$-up, as it was suggested by Liou in [11] to address the problem of entropy preservation.

- 'JCAM 2013': The transporting face velocity is

$$
\begin{aligned}
v_{1 / 2}^{\mathrm{T}}=c_{1 / 2} M_{1 / 2}-\frac{K_{p}}{\bar{\varrho}\left[f_{c}\left(M_{0}\right) c_{1 / 2}+\frac{\beta}{\Delta t / \Delta x}\right]} \max \left\{1-\sigma \bar{M}^{2}, 0\right\} & \\
& \times\left(p_{\mathrm{R}}-p_{\mathrm{L}}\right)
\end{aligned}
$$

where $\beta \in O(1)$. The face pressure is identical to the one of the $\mathrm{AUSM}^{+}$scheme.

\section{A.2 SLAU $[4,21]$}

- Mass flux:

$$
(\varrho v)_{1 / 2}=\frac{1}{2}\left[\varrho_{\mathrm{L}}\left(V_{n}^{+}+\left|\bar{V}_{n}\right|^{+}\right)+\varrho_{\mathrm{R}}\left(V_{n}^{-}-\left|\bar{V}_{n}\right|^{-}\right)\right]-\frac{\chi\left(\widetilde{M}_{1 / 2}\right)}{2 c_{1 / 2}} \Delta p
$$

where

$$
\begin{aligned}
& \left|\bar{V}_{n}\right|^{+}=(1-g)\left|\bar{V}_{n}\right|+g\left|V_{n}^{+}\right|, \quad\left|\bar{V}_{n}\right|^{-}=(1-g)\left|\bar{V}_{n}\right|+g\left|V_{n}^{-}\right| \\
& \left|\bar{V}_{n}\right|=\frac{\varrho_{\mathrm{L}}\left|V_{n}^{+}\right|+\varrho_{\mathrm{R}}\left|V_{n}^{-}\right|}{\varrho_{\mathrm{L}}+\varrho_{\mathrm{R}}} \\
& g=-\max \left\{\min \left\{M_{\mathrm{L}}, 0\right\},-1\right\} \min \left\{\max \left\{M_{\mathrm{R}}, 0\right\}, 1\right\} \\
& c_{1 / 2}=\frac{c_{\mathrm{L}}+c_{\mathrm{R}}}{2} \\
& \chi(M)=(1-M)^{2}, \widehat{M}_{1 / 2}=\min \left\{1, \frac{1}{c_{1 / 2}} \sqrt{\frac{u_{\mathrm{L}}^{2}+v_{\mathrm{L}}^{2}+w_{\mathrm{L}}^{2}+u_{\mathrm{R}}^{2}+v_{\mathrm{R}}^{2}+w_{\mathrm{R}}^{2}}{2}}\right\}
\end{aligned}
$$

- Face pressure ('SLAU 2' version proposed in [4]):

$$
\begin{aligned}
& p_{1 / 2}=\frac{p_{\mathrm{L}}+p_{\mathrm{R}}}{2}+\frac{f_{p, 0}^{+}\left(M_{\mathrm{L}}\right)-f_{p, 0}^{-}\left(M_{\mathrm{R}}\right)}{2}\left(p_{\mathrm{L}}-p_{\mathrm{R}}\right) \\
& +\sqrt{\frac{u_{\mathrm{L}}^{2}+v_{\mathrm{L}}^{2}+w_{\mathrm{L}}^{2}+u_{\mathrm{R}}^{2}+v_{\mathrm{R}}^{2}+w_{\mathrm{R}}^{2}}{2}}\left(f_{p, 0}^{+}\left(M_{\mathrm{L}}\right)+f_{p, 0}^{-}\left(M_{\mathrm{R}}\right)-1\right) \bar{\varrho} c_{1 / 2}
\end{aligned}
$$

where $\bar{\varrho}=\frac{1}{2}\left(\varrho_{\mathrm{L}}+\varrho_{\mathrm{R}}\right)$ 


\section{A.3 Momentum interpolation (MI) [15,16]}

- Mass flux (1-D version and velocity is assumed to be positive):

$$
(\varrho v)_{i+1 / 2}=\frac{B_{i+1 / 2}}{A_{i+1 / 2}}-\frac{1}{A_{i+1 / 2}}\left(p_{i+1}-p_{i}\right)-\frac{\Delta x}{A_{i+1 / 2}} \mathrm{~d}_{t}(\varrho v)_{i+1 / 2} .
$$

In this expression,

$$
\frac{B_{i+1 / 2}}{A_{i+1 / 2}}=\frac{1}{2}\left(\frac{B_{i}}{A_{i}}+\frac{B_{i+1}}{A_{i+1}}\right)
$$

where $A_{i}$ and $B_{i}$ are terms in the momentum equation of node $i$ according to

$$
B_{i}=A_{i}(\varrho v)_{i}+p_{i+1 / 2}-p_{i-1 / 2}+\Delta x \mathrm{~d}_{t}(\varrho v)_{i}
$$

and

$$
\frac{1}{A_{i+1 / 2}}=\frac{1}{2}\left(\frac{1}{A_{i}}+\frac{1}{A_{i+1}}\right)
$$

There, $A_{i}=v_{i+1 / 2}$ and $A_{i+1}=v_{i+3 / 2}$, taken at the previous iteration or time-step and defined with the $\mathrm{AUSM}^{+}$expression.

- Face pressure. In all the presented calculations with momentum interpolation, the face pressure is the one of the $\mathrm{AUSM}^{+}$scheme:

$$
p_{1 / 2}=f_{p, 3 / 16}^{+}\left(M_{\mathrm{L}}\right) p_{\mathrm{L}}+f_{p, 3 / 16}^{-}\left(M_{\mathrm{R}}\right) p_{\mathrm{R}}
$$

\section{Appendix B Pressure correction algorithm [15]}

To simplify the presentation, the flow is 1-D and the velocity is positive. Each timestep $n \rightarrow n+1$ is decomposed into iterations denoted by the superscript $k$. At the first iteration of the time-step $n$, one has $k=n$. The superscripts $\star \star, \star$ and / denote 'pre-predicted', predicted and corrected quantities of each iteration $k . \psi$ denotes the slope limiter. Practically, no more than five iterations are allowed, and the so-called Bounded Central slope limiter was chosen in the unsteady low Mach number flow calculations. The ratio $\Delta t / \Delta x$ is denoted by $\tau$. 


\section{B.1 'Pre-prediction' step: Construction of the common transporting velocity}

- $\varrho_{i}^{\star \star}$ from

$$
\begin{aligned}
\frac{1}{2 \tau}\left(3 \varrho_{i}^{\star \star}-4 \varrho_{i}^{n}+\varrho_{i}^{n-1}\right)+ & {\left[\varrho_{i}^{\star \star}+\frac{1}{2} \psi_{i}\left(\varrho^{k}\right)\left(\varrho_{i}^{k}-\varrho_{i-1}^{k}\right)\right] v_{i+1 / 2}^{k} } \\
& -\left[\varrho_{i-1}^{\star \star}+\frac{1}{2} \psi_{i-1}\left(\varrho^{k}\right)\left(\varrho_{i-1}^{k}-\varrho_{i-2}^{k}\right)\right] v_{i-1 / 2}^{k}=0
\end{aligned}
$$

- $(\varrho v)_{i}^{\star \star}=\varrho_{i}^{\star \star} v_{i}^{k},(\varrho E)_{i}^{\star \star}=\frac{p_{i}^{k}}{\gamma-1}+\frac{1}{2} \varrho_{i}^{\star \star}\left(v_{i}^{k}\right)^{2}$

- Transporting face velocity:

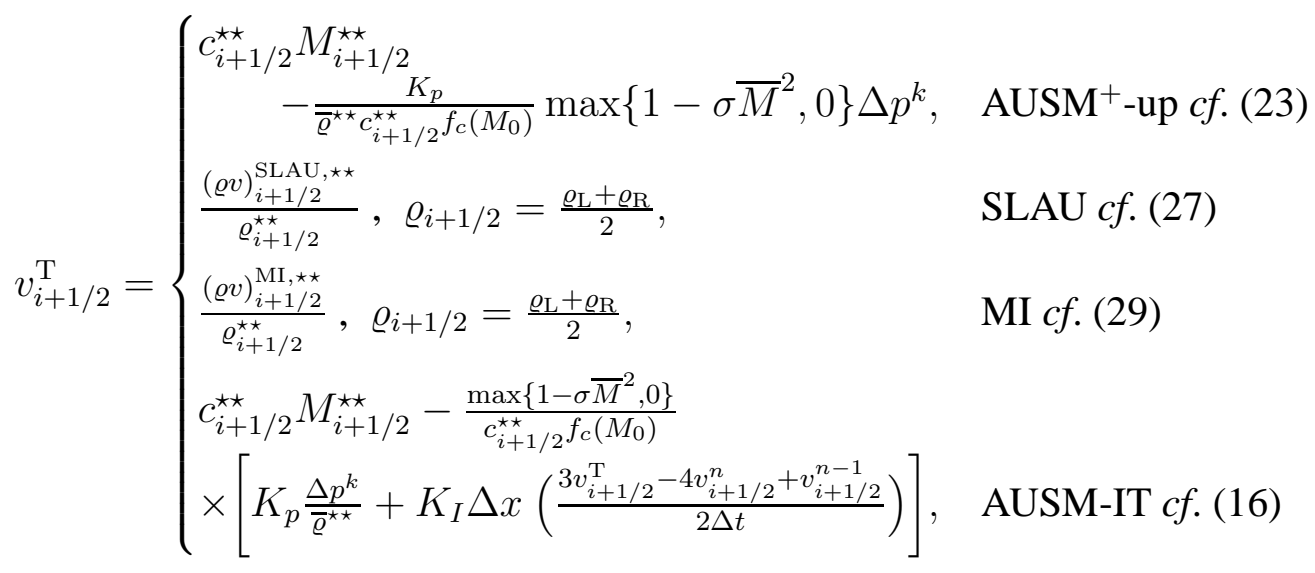

\section{B.2 Prediction step}

- $p_{i}^{\star}=p_{i}^{k}$

- $\varrho_{i}^{\star}$ from

$$
\begin{aligned}
\frac{1}{2 \tau}\left(3 \varrho_{i}^{\star}-4 \varrho_{i}^{n}+\varrho_{i}^{n-1}\right)+\left[\varrho_{i}^{\star}\right. & \left.+\frac{1}{2} \psi_{i}\left(\varrho^{k}\right)\left(\varrho_{i}^{k}-\varrho_{i-1}^{k}\right)\right] v_{i+1 / 2}^{\mathrm{T}} \\
- & {\left[\varrho_{i-1}^{\star}+\frac{1}{2} \psi_{i-1}\left(\varrho^{k}\right)\left(\varrho_{i-1}^{k}-\varrho_{i-2}^{k}\right)\right] v_{i-1 / 2}^{\mathrm{T}}=0 }
\end{aligned}
$$


- $(\varrho v)_{i}^{\star}$ from

$$
\begin{aligned}
& \frac{1}{2 \tau}\left[3(\varrho v)_{i}^{\star}-4(\varrho v)_{i}^{n}+(\varrho v)_{i}^{n-1}\right] \\
& +\left[(\varrho v)_{i}^{\star}+\frac{1}{2} \psi_{i}\left((\varrho v)^{k}\right)\left[(\varrho v)_{i}^{k}-(\varrho v)_{i-1}^{k}\right]\right] v_{i+1 / 2}^{\mathrm{T}} \\
& -\left[(\varrho v)_{i-1}^{\star}+\frac{1}{2} \psi_{i-1}\left((\varrho v)^{k}\right)\left[(\varrho v)_{i-1}^{k}-(\varrho v)_{i-2}^{k}\right]\right] v_{i-1 / 2}^{\mathrm{T}} \\
& +p_{i+1 / 2}^{k}-p_{i-1 / 2}^{k}=0
\end{aligned}
$$

- $(\varrho E)_{i}^{\star}=\frac{p_{i}^{k}}{\gamma-1}+\frac{1}{2} \frac{\left[(\varrho v)_{i}^{\star}\right]^{2}}{\varrho_{i}^{\star}},(\varrho H)_{i}^{\star}=(\varrho E)_{i}^{\star}+p_{i}^{k}$

\section{B.3 Correction step}

- $p_{i}^{\prime}$ from

$$
\frac{1}{2 \tau}\left[3(\varrho E)_{i}^{k+1}-4(\varrho E)_{i}^{n}+(\varrho E)_{i}^{n-1}\right]+(\varrho v H)_{i+1 / 2}^{k+1}-(\varrho v H)_{i-1 / 2}^{k+1}=0
$$

where

$$
\begin{aligned}
& (\varrho v H)_{i+1 / 2}^{k+1}=(\varrho H)_{i+1 / 2}^{\star} v_{i+1 / 2}^{\mathrm{T}}+H_{i+1 / 2}^{\star}(\varrho v)_{i+1 / 2}^{\prime}+(\varrho H)_{i+1 / 2}^{\prime} v_{i+1 / 2}^{\mathrm{T}} \\
& (\varrho H)_{i+1 / 2}^{\star}, \quad H_{i+1 / 2}^{\star}: \text { upwinded in second-order accurate form } \\
& (\varrho H)_{i+1 / 2}^{\prime}=\frac{\gamma}{\gamma-1} p_{i+1 / 2}^{\prime} \\
& p_{i+1 / 2}^{\prime}=f_{p, 3 / 16}^{+}\left(M_{i}^{\star}\right) p_{i}^{\prime}+f_{p, 3 / 16}^{-}\left(M_{i+1}^{\star}\right) p_{i+1}^{\prime} \\
& (\varrho v)_{i+1 / 2}^{\prime}=(\varrho v)_{i+1 / 2, \text { Momentum }}^{\prime}+(\varrho v)_{i+1 / 2, \text { Flux }}^{\prime} \\
& (\varrho v)_{i+1 / 2, \text { Momentum }}^{\prime}=-\frac{2}{3} \tau\left(p_{i+1}^{\prime}-p_{i}^{\prime}\right) \quad(\text { SIMPLE approximation }) \\
& (\varrho v)_{i+1 / 2, \text { Flux }}^{\prime}=-\kappa_{i+1 / 2}\left(p_{i+1}^{\prime}-p_{i}^{\prime}\right) \\
& \kappa_{i+1 / 2}= \begin{cases}\frac{K_{p}}{\varrho_{i+1 / 2}^{\star} c_{i+1 / 2}^{\star} f\left(M_{0}^{\star}\right)} \\
\times \max \left\{1-\left(M_{i+1 / 2}^{\star}\right)^{2} \sigma, 0\right\} \varrho_{L}^{\star}, & \text { AUSM }{ }^{+} \text {-up } c f .(23) \\
\frac{\chi_{i+1 / 2}}{c_{i+1 / 2}}, & \text { SLAU } c f . \text { (27) } \\
0, & \text { MI } c f . \text { (29) or AUSM-IT } c f . \text { (16) }\end{cases}
\end{aligned}
$$


- $(\varrho v)_{i}^{\prime}$ from

$$
\begin{aligned}
& \frac{3}{2}(\varrho v)_{i}^{\prime}=-\tau\left[\left\{(\varrho v)_{i}^{\prime}+\frac{1}{2} \psi_{i}\left((\varrho v)^{k}\right)\left[(\varrho v)_{i}^{\prime}-(\varrho v)_{i-1}^{\prime}\right]\right\} v_{i+1 / 2}^{\mathrm{T}}\right. \\
& \left.-\left\{(\varrho v)_{i-1}^{\prime}+\frac{1}{2} \psi_{i-1}\left((\varrho v)^{k}\right)\left[(\varrho v)_{i-1}^{\prime}-(\varrho v)_{i-2}^{\prime}\right]\right\} v_{i-1 / 2}^{\mathrm{T}}\right] \\
& -\tau\left(p_{i+1 / 2}^{\prime}-p_{i-1 / 2}^{\prime}\right)
\end{aligned}
$$

\section{B.4 Updates}

- Cell quantities:

$$
\begin{aligned}
& p_{i}^{k+1}=p_{i}^{k}+p_{i}^{\prime} \quad, \quad \varrho_{i}^{k+1}=\varrho_{i}^{\star}\left(1+\frac{p_{i}^{\prime}}{p_{i}^{k}}\right) \quad, \quad(\varrho v)_{i}^{k+1}=(\varrho v)_{i}^{\star}+(\varrho v)_{i}^{\prime} \\
& (\varrho E)_{i}^{k+1}=(\varrho E)_{i}^{\star}+\frac{p_{i}^{\prime}}{\gamma-1} \quad, \quad(\varrho H)_{i}^{k+1}=(\varrho E)_{i}^{k+1}+p_{i}^{k+1}
\end{aligned}
$$

- Cell-face quantities:

$$
p_{i+1 / 2}^{k+1}=\left\{\begin{array}{cc}
f_{p, \alpha}^{+}\left(M_{\mathrm{L}}^{k+1}\right) p_{\mathrm{L}}^{k+1}+f_{p, \alpha}^{-}\left(M_{\mathrm{R}}^{k+1}\right) p_{\mathrm{R}}^{k+1} & \\
-K_{v} f_{p, \alpha}^{+}\left(M_{\mathrm{L}}^{k+1}\right) f_{p, \alpha}^{-}\left(M_{\mathrm{R}}^{k+1}\right) & \\
\times\left(\varrho_{\mathrm{L}}^{k+1}+\varrho_{\mathrm{R}}^{k+1}\right) f_{c}\left(M_{0}\right) & \\
\times c_{i+1 / 2}^{k+1}\left(v_{\mathrm{R}}^{k+1}-v_{\mathrm{L}}^{k+1}\right), & \text { AUSM }^{+}-\text {up } c f . \text { (25) } \\
\frac{p_{\mathrm{L}}^{k+1}+p_{\mathrm{R}}^{k+1}}{2}+\frac{f_{p, 0}^{+}\left(M_{\mathrm{L}}^{k+1}\right)-f_{p, 0}^{-}\left(M_{\mathrm{R}}^{k+1}\right)}{2} & \\
\times\left(p_{\mathrm{L}}^{k+1}-p_{\mathrm{R}}^{k+1}\right)+\sqrt{\frac{\left(v_{\mathrm{L}}^{k+1}\right)^{2}+\left(v_{\mathrm{R}}^{k+1}\right)^{2}}{2}} & \\
\times\left(f_{p, 0}^{+}\left(M_{\mathrm{L}}^{k+1}\right)+f_{p, 0}^{-}\left(M_{\mathrm{R}}^{k+1}\right)-1\right) & \\
\times \bar{\varrho}^{k+1} c_{i+1 / 2}^{k+1}, & \text { SLAU } c f .(28) \\
f_{p, \alpha}^{+}\left(M_{\mathrm{L}}^{k+1}\right) p_{\mathrm{L}}^{k+1}+f_{p, \alpha}^{-}\left(M_{\mathrm{R}}^{k+1}\right) p_{\mathrm{R}}^{k+1}, & \text { AUSM-IT } c f . \text { (17) or MI }
\end{array}\right.
$$

For any used numerical flux:

$$
v_{i+1 / 2}^{k+1}=c_{i+1 / 2}^{k+1} M_{i+1 / 2}^{k+1}
$$

with $c_{1 / 2}$ and $M_{1 / 2}$ defined in Appendix A. Note that this cell-face velocity is the $\mathrm{AUSM}^{+}$cell-face velocity [10]. 


\section{References}

[1] S. Dellacherie. Analysis of Godunov type schemes applied to the compressible Euler system at low Mach number. J. Comput. Phys., 229:978-1016, 2010.

[2] H. Guillard and A Murrone. On the behavior of upwind schemes in the low Mach number limit: II. Godunov type schemes. Comput. Fluids, 33:655-675, 2004.

[3] H. Guillard and C. Viozat. On the behavior of upwind schemes in the low Mach number limit. Comput. Fluids, 28:63-86, 1999.

[4] K. Kitamura and E. Shima. Towards shock-stable and accurate hypersonic heating computations: A new pressure flux for AUSM-family schemes. J. Comput. Phys., 245:62-83, 2013.

[5] S. Klainerman and A. Majda. Compressible and incompressible fluids. Comm. Pure Appl. Math., 35:629-651, 1982.

[6] R. Klein. Semi-Implicit Extension of a Godunov-Type Scheme Based on Low Mach Number Asymptotics I: One-Dimensional Flow. J. Comput. Phys., 121:213-237, 1995.

[7] P. D. Lax. Weak solutions of nonlinear hyperbolic equations and their numerical computation. Commun. Pure Appl. Math., 7:159-193, 1954.

[8] X. S. Li and C. W. Gu. An All-Speed Roe-type scheme and its asymptotic analysis of low Mach number behaviour. J. Comput. Phys., 227:5144-5159, 2008.

[9] X. S. Li and C. W. Gu. Mechanism of Roe-type schemes for all-speed flows and its application. Comput. Fluids, 86:56-70, 2013.

[10] M.-S. Liou. A sequel to AUSM: AUSM+ ${ }^{+}$J. Comput. Phys., 129:364-382, 1996.

[11] M.-S. Liou. A sequel to AUSM, part II: AUSM ${ }^{+}$-up for all speeds. J. Comput. Phys., 214:137-170, 2006.

[12] M.-S. Liou and C. J. Steffen. A new flux splitting scheme. J. Comput. Phys., 107:2339, 1993.

[13] A. Meister. Asymptotic Single and Multiple Scale Expansions in the Low Mach Number Limit. SIAM J. Appl. Math., 60(1):256-271, 1999.

[14] Y. Moguen, E. Dick, J. Vierendeels, and P. Bruel. Pressure-velocity coupling for unsteady low Mach number flow simulations: An improvement of the $\mathrm{AUSM}^{+}$-up scheme. J. Comput. Appl. Math., 246:136-143, 2013.

[15] Y. Moguen, T. Kousksou, P. Bruel, J. Vierendeels, and E. Dick. Pressure-velocity coupling allowing acoustic calculation in low Mach number flow. J. Comput. Phys., 231:5522-5541, 2012.

[16] A. Pascau. Cell face velocity alternatives in a structured colocated grid for the unsteady Navier-Stokes equations. Int. J. Numer. Meth. Fluids, 65:812-833, 2011. 
[17] C. M. Rhie and W. L. Chow. Numerical Study of the Turbulent Flow Past an Airfoil with Trailing Edge Separation. AIAA J., 21(11):1525-1532, 1983.

[18] F. Rieper. A low-Mach number fix for Roe's approximate Riemann solver. J. Comput. Phys., 230:5263-5287, 2011.

[19] J. Sachdev, A. Hosangadi, and V. Sankaran. Improved flux formulations for unsteady low Mach number flows. AIAA Paper No 2012-3067, $42^{\text {nd }}$ AIAA Fluid Dynamics Conference, New Orleans, United States of America, June 25-28, 2012.

[20] E. Shima. On the improvement of the all-speed flux scheme for very low Mach number flows. AIAA Paper No 2013-2696, $21^{\text {st }}$ AIAA Computational Fluid Dynamics Conference, San Diego, United States of America, June 24-27, 2013.

[21] E. Shima and K. Kitamura. Parameter-free simple low-dissipation AUSM-family scheme for all speeds. AIAA J., 49(8):1693-1709, 2011.

[22] L. L. Takacs. A two-step scheme for the advection equation with minimized dissipation and dispersion errors. Mon. Wea. Rev., 113:1050-1065, 1985.

[23] H. Tang. On the sonic point glitch. J. Comput. Phys., 202:507-532, 2005.

[24] E. Toro. Riemann solvers and numerical methods for fluid dynamics - A practical introduction. Springer, third edition, 2009. 